\title{
Analysis approaches to address treatment nonadherence in pragmatic trials with point-treatment settings: a simulation study
}

\author{
Md. Belal Hossain', Lucy Mosquera² and Mohammad Ehsanul Karim 1,3*
}

\begin{abstract}
Background: Two-stage least square [2SLS] and two-stage residual inclusion [2SRI] are popularly used instrumental variable (IV) methods to address medication nonadherence in pragmatic trials with point treatment settings. These methods require assumptions, e.g., exclusion restriction, although they are known to handle unmeasured confounding. The newer IV-method, nonparametric causal bound [NPCB], showed promise in reducing uncertainty compared to usual IV-methods. The inverse probability-weighted per-protocol [IP-weighted PP] method is useful in the same setting but requires different assumptions, e.g., no unmeasured confounding. Although all of these methods are aimed to address the same nonadherence problem, comprehensive simulations to compare performances of them are absent in the literature.

Methods: We performed extensive simulations to compare the performances of the above methods in addressing nonadherence when: (1) exclusion restriction satisfied and no unmeasured confounding, (2) exclusion restriction is met but unmeasured confounding present, and (3) exclusion restriction is violated. Our simulations varied parameters such as, levels of adherence rates, unmeasured confounding, and exclusion restriction violations. Risk differences were estimated, and we compared performances in terms of bias, standard error (SE), mean squared error (MSE), and 95\% confidence interval coverage probability.

Results: For setting (1), 2SLS and 2SRI have small bias and nominal coverage. IP-weighted PP outperforms these IV-methods in terms of smaller MSE but produces high MSE when nonadherence is very high. For setting (2), IP-weighted-PP generally performs poorly compared to 2SLS and 2SRI in term of bias, and both-stages adjusted IV-methods improve precision than naive IV-methods. For setting (3), IV-methods perform worst in all scenarios, and (Continued on next page)
\end{abstract}

${ }^{*}$ Correspondence: ehsan.karim@ubc.ca

'School of Population and Public Health, University of British Columbia, Vancouver, BC, Canada

${ }^{3}$ Centre for Health Evaluation and Outcome Sciences, St. Paul's Hospital,

Vancouver, BC, Canada

Full list of author information is available at the end of the article

(C) The Author(s). 2022 Open Access This article is licensed under a Creative Commons Attribution 4.0 International License, which permits use, sharing, adaptation, distribution and reproduction in any medium or format, as long as you give appropriate credit to the original author(s) and the source, provide a link to the Creative Commons licence, and indicate if changes were made. The images or other third party material in this article are included in the article's Creative Commons licence, unless indicated otherwise in a credit line to the material. If material is not included in the article's Creative Commons licence and your intended use is not permitted by statutory regulation or exceeds the permitted use, you will need to obtain permission directly from the copyright holder. To view a copy of this licence, visit http://creativecommons.org/licenses/by/4.0/. The Creative Commons Public Domain Dedication waiver (http://creativecommons.org/publicdomain/zero/1.0/) applies to the data made available in this article, unless otherwise stated in a credit line to the data. 
(Continued from previous page) IP-weighted-PP produces unbiased estimates and small MSE when confounders are adjusted. NPCB produces larger uncertainty bound width in almost all scenarios. We also analyze a two-arm trial to estimate vitamin-A supplementation effect on childhood mortality after addressing nonadherence.

Conclusions: Understanding finite sample characteristics of these methods will guide future researchers in determining suitable analysis strategies. Since assumptions are different and often untestable for IP-weighted PP and IV methods, we suggest analyzing data using both IP-weighted PP and IV approaches in search of a robust conclusion.

Keywords: Pragmatic trials, Nonadherence, Simulation, Unmeasured confounding

\section{Background}

Randomization is the core principle of clinical trials, that protects against noncomparability between treatment groups at baseline. Pragmatic trials are getting popular for exploring the effectiveness of treatments in settings that mimic real-world clinical practice [1]. In this manuscript, we focus on nonadherence in a point-treatment setting, where treatment is assigned at baseline shortly after randomization. Although the treatments are randomly assigned at baseline for pragmatic trials, it is possible that some subjects may deviate from the protocol because of switching to other treatments, loss to follow-up due to side-effects, etc. In the presence of treatment nonadherence, a treatment effect estimate that is agnostic to the adherence pattern, is less useful for the patients and caregivers to make a decision about the treatment. Moreover, pragmatic trials are often unblinded [2], which often introduces selection bias [3]. Typically, those adherent and nonadherent subjects are different in terms of prognostic factors [4], and it is necessary to take into account those factors while estimating the treatment effect. An additional challenge arises when adherence depends on subject's char measured during baseline. Inability to adjust for the unmeasured confounding could bias the treatment effect, depending on the method of estimation $[4,5]$.

An intention-to-treat (ITT) analysis is treated as a default analytic technique to address nonadherence in randomized and pragmatic trial settings [6]. The baseline randomization preserved by this analysis, and hence baseline confounding is not a concern $[7,8]$. The per-protocol (PP) and as-treated (AT) are two other common methods that are popularly used to address nonadherence $[9$, 10]. In practice, all these naive methods usually produce biased estimates if the nonadherence occurs in a nonrandom fashion [4]. Typically that is the case, if some of the characteristics of the patient act as a confounder, e.g., are predictive of the nonadherence pattern as well as the outcome of interest. Particularly for PP approaches, when subjects who deviate from protocol are removed from the analysis, comparability of the subjects in both arms ensured by the process of randomization is violated. In that case, baseline confounder adjusted-PP methods are utilized to address the nonadherence in pragmatic trials, if the baseline measurements of the necessary confounders are available [7, 11-13]. As an alternative, inverse probability (IP)-weighted PP is also used to produce marginal estimates, and that can adjust for the measured confounders. However, if some of the necessary confounders are not measured, the PP based methods usually provide biased estimates [14]. In that case, the instrumental variable (IV)-based methods can still be used to get the unbiased estimate of the treatment effect [15], which is a known strength of these IV methods. Previous studies used various versions of IV-based methods to address the nonadherence in the pragmatic trial settings $[10,16$, 17]. Two-Stage least squares (2SLS), two-stage residual inclusion (2SRI) are well-known IV-based methods, with a known limitation that they are usually inefficient. Newer IV-based method, nonparametric causal bound (NPCB), is proposed in the literature which uses a partial identification approach, but provides only bounds rather than point estimates [18]. This method was touted as a promising method in terms of reducing the levels of uncertainty, but the original method can not adjust for any confounders. Two previous studies analyzed data from a two-arm randomized control trial in northern Sumatra using this NPCB method $[19,20]$. They reported very wide bounds from NPCB method. As a motivating example, we used the same dataset to explore the performance of two other IV methods in the same scenario, and from the simulations under various parameter spaces, we investigated the possible reasons of why the NPCB may have produced wide bounds in this study.

Both the adjusted PP methods (baseline adjusted and IP-weighted) and IV-based methods (2SLS, 2SRI, and NPCB) aim to deal with adherence adjustment but require different assumptions. For example, adjusted PP approaches assume there is no unmeasured confounding, while the IV-based methods assume the exclusion restriction (the IV is associated with the outcome only through the treatment) [21-23]. These two assumptions cannot be empirically verified given the observed data, but the violation of the assumptions can lead to biased estimates $[14,24]$. Let we are interested in estimating the effect of a heart transplant on one-year mortality. No unmeasured confounding implies that all necessary confounders (e.g., variables causally associated with both heart transplant and mortality) are measured. If randomization is 
the instrument, the exclusion restriction assumption suggests that randomization should not directly influence any variable other than whether patients did the heart transplant versus standard care. Despite the different statistical assumptions required by these two classes of methods dealing with the same nonadherence problem, the comprehensive comparison of these adherence-adjusted methods remains largely absent in the literature. Moreover, there are two definitions of PP methods, such as (a) censoring those patients if and when deviating from the protocol and (b) excluding those patients entirely from the analysis [25]. There exist some recent simulations in the literature for the first definition $[26,27]$ but we could not find much explorations for the second definition. Besides, NPCB being a relatively newly proposed method, finite sample characteristics of its estimates under the above settings should be explored.

In the present study, we aimed to compare two adjusted PP approaches and three versions of the IV-based methods in the presence of nonadherence. To explore the benefits and limitations of the applications of these approaches (along with the naive approaches), and to evaluate how robust these methods are if the respective assumptions are violated, we propose comprehensive simulation studies to compare these methods under different settings, such as when (1) exclusion restriction satisfied and no unmeasured confounding, (2) exclusion restriction satisfied but unmeasured confounding present, and (3) exclusion restriction violated. Under these scenarios, we try to identify which methods are more appropriate to use.

\section{Methods}

\section{Estimation methods}

\section{Methods}

We compared the estimates of the following methods for a binary outcome of interest: naive methods (ITT, naive PP, and naive AT), two PP methods (baseline adjusted PP and IP-weighted PP), and three classes of IV-based methods (2SLS, 2SRI, and NPCB). Some earlier studies [28, 29] used the 2SLS method where confounders are adjusted only in the first stage of the model. Wang et al. [29] used the 2SRI method with confounders adjusted only in the first stage of the model. To compare whether there are any effects of considering different versions of these IV methods, we have added some variations of all these methods, such as the naive, first-stage adjusted, and both-stages adjusted of 2SLS and 2SRI approaches. A brief description of these models is provided in Table 1, and the full description can be found in Appendix A.

\section{Assumptions}

The key assumption for the PP methods and IV-based methods is the no unmeasured confounding and exclusion restriction, respectively [21-23]. The other assumptions of all the methods are provided in Table 2 and are summarized in Appendix A.

Confounders, confounding, and exclusion restriction are the three important concepts to understand the simulation settings we describe below. The traditional definition of a "confounder" is that it meets all three conditions: the variable is (i) causally associated with the outcome, (ii) non-causally or causally associated with the treatment, and (iii) not in the causal pathway between the treatment and outcome [32]. Consider the causal diagram is shown in Fig. 1(A), where $Z$ is the randomization, $A$ is the treatment, and $Y$ is the outcome. Consider two variables in the figure: $L$ (measured) and $U$ (unmeasured). Both $L$ and $U$ are (i) causally associated with $Y$, (ii) causally associated with $A$, and (iii) not in the causal pathway between $A$ and $Y$ (i.e., not mediators). $L$ and $U$ are also not colliders (i.e., not common effect of $A$ and $Y$ ) [33], but confounders in this example. On the other hand, "confounding" is a fundamental concept in epidemiological studies to estimate causal effects. No unmeasured confounding implies that the distribution of $Y$ would be the same for the treated and untreated subjects if both received the same treatment. That means, the treated and untreated subjects are comparable in terms of any measured and unmeasured factors if both had been treated [33]. To address confounding, in the causal diagram terminology, we must close all open "backdoor paths" between $A$ and $Y$ (e.g., adjust for those variables in the regression model that block the open backdoor paths) [33, 34]. There could be scenarios where more than one variable lies along a backdoor path. In such a scenario, adjusting for a single confounder on the path can be sufficient to block the backdoor path $[33,34]$. For example, in Fig. 1(B), although we have two "confounders" ( $L$ and $U$ ) by the conventional confounder definition $(A \leftarrow L \rightarrow Y$, and $A \leftarrow L \leftarrow U \rightarrow Y$ ), adjusting only for $L$ is sufficient to close all open backdoor paths between $A$ and $Y$. That means, $L$ constitutes the minimal sufficient adjustment set [35]. In such a case, adjusting only for $L$ can address "confounding" (even though there remained $U$ unadjusted, which is a confounder by conventional definition), and it is certainly possible to get an unbiased effect of $A$ on $Y$ [33], irrespective of whether we can measure or adjust $U$. But in Fig. 1(C), adjusting only for $L$ is not sufficient to close all open backdoor paths between $A$ and $Y$ (since a path is open through $U$ ), indicating the presence of confounding in the $A-Y$ relationship. For more discussion on confounders and confounding, please see [32, 33].

The exclusion restriction assumption says the instrument is associated with the outcome only through the treatment. For example, in Figs. 1(B)-(C), the instrument $Z$ is associated with $Y$ only through its effect on $A$, meaning that the exclusion restriction is met [36]. As shown in Fig. 1(D), if the instrument influences $Y$ through a path- 
Table 1 Description of the estimation methods used for dealing with treatment nonadherence in pragmatic trials with point-treatment settings

\begin{tabular}{|c|c|}
\hline Name of the method & Description \\
\hline \multicolumn{2}{|l|}{ Naïve methods } \\
\hline ITT & $\begin{array}{l}\text { It models the randomization variable }(Z) \text { on the outcome }(Y) \text { without adjustment for measured confounders } L \text {. This } \\
\text { method does not consider whether individuals adhered to the treatment }[6] \text {. }\end{array}$ \\
\hline Naïve PP & $\begin{array}{l}\text { It models } Z \text { on } Y \text { among those subjects who receive the treatment according to the protocol but without adjustment } \\
\text { for } L \text {. This method excludes those subjects who deviated from the protocol. }\end{array}$ \\
\hline Naïve AT & $\begin{array}{l}\text { It models the treatment actually received }(A) \text { on } Y \text { without adjustment for } L \text {. This method does not consider whether } \\
\text { individuals randomized to the treatment groups. }\end{array}$ \\
\hline \multicolumn{2}{|l|}{ Adjusted methods } \\
\hline Baseline-adjusted ITT & The same as ITT but it adjusts for $L$. \\
\hline Baseline-adjusted PP & The same as naïve PP but it adjusts for $L$. \\
\hline IP-weighted PP & $\begin{array}{l}\text { This method creates inverse probability adherence weights to generate a pseudo population to estimate the treat- } \\
\text { ment effect by removing the effect of nonadherence [25]. We used a logistic model that adjusts for } L \text { to estimate } \\
\text { the probabilities, and then used the marginal structural model to estimate the parameters of interest. The stabilized } \\
\text { weights were used to prevent from extreme weights }[7,30] \text {. }\end{array}$ \\
\hline
\end{tabular}

IV-methods

Naïve 2SLS

The instrument $(Z)$ is modelled to the treatment $(A)$ in the first stage, and then the predicted treatment is modelled to the outcome $(Y)$ in the second stage [31]. There was no adjustment for $L$ in either stage of the model.

First-stage adjusted 2SLS

The same as naive 2SLS except it adjusts for $L$ in the first stage of the model $[28,29]$.

Both-stages adjusted 2SLS

The same as naive 2SLS except it adjusts for $L$ in both stages of the model.

Naïve 2SRI

The instrument $(Z)$ is modelled to the treatment variable $(A)$ in the first stage, and then the residuals from the first stage and the treatment variable are modelled to the outcome $(Y)$ in the second stage [22]. There was no adjustment for $L$ in either stage of the model.

First-stage adjusted 2SRI

Both-stages adjusted 2SRI

The same as naive 2SRI except it adjusts for $L$ in the first stage of the model [29].

NPCB

The same as naive 2SRI except it adjusts for $L$ in both stages of the model [22].

This nonparametric method uses a constrained probability statement to provide bounds on the estimated treatment effect rather than a point estimate [18, 19].

Note: The 2SLS, 2SRI, and NPCB are IV-based methods. Whether there is any adjustment for covariates, the 2SLS/2SRI are not termed as the naive, first-stage adjusted, or both-stages adjusted 2SLS/2SRI in the literature. For comparison purposes, we termed these methods as the naive, first-stage adjusted, or both-stages adjusted 2SLS/2SRI; Abbreviations: ITT: intention-to-treat; PP: per-protocol; AT: as-treated; IP-weighted PP: inverse probability weighted per-protocol; 2SLS: two-stage least square; 2SRI: two-stage residual inclusion model; NPCB: non-parametric causal bound.

Table 2 Assumptions of the estimation methods described in Table 1 that are used for addressing treatment nonadherence in pragmatic trials with point-treatment settings

Name of the method Key assumption Other assumptions

ITT, baseline-adjusted ITT, naïve PP, naïve AT

Baseline-adjusted PP, IP-weighted PP

Naïve 2SLS, first-stage adjusted 2SLS, both-stages adjusted 2SLS

Naïve 2SRI, first-stage adjusted 2SRI, both-stages adjusted 2SRI

NPCB
No unmeasured confounding

No unmeasured confounding

Exclusion restriction

Exclusion restriction

Exclusion restriction
Consistency, no interference, positivity, nonadherence occurred completely at random

Consistency, no interference, positivity, correct model specification

Consistency, no interference, positivity, correct model specification, relevance, monotonicity

Consistency, no interference, positivity, correct model specification, relevance, monotonicity, linearity of residuals

Consistency, no interference, positivity, relevance, monotonicity

Abbreviations: ITT: intention-to-treat; PP: per-protocol; AT: as-treated; IP-weighted PP: inverse probability weighted per-protocol; 2SLS: two-stage least square; 2SRI: two-stage residual inclusion model; NPCB: non-parametric causal bound. 


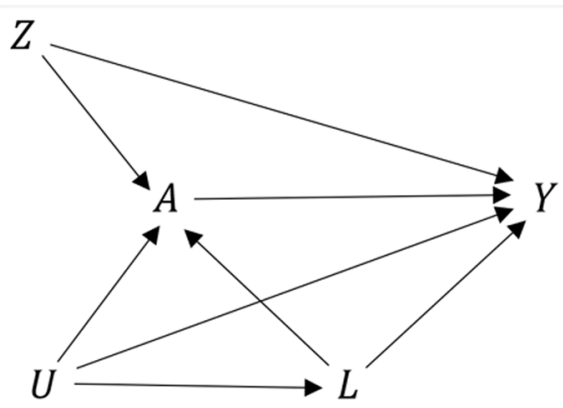

(A) Main DAG

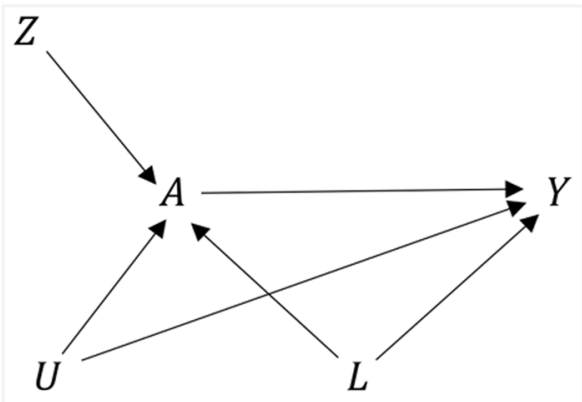

(C) DAG 2

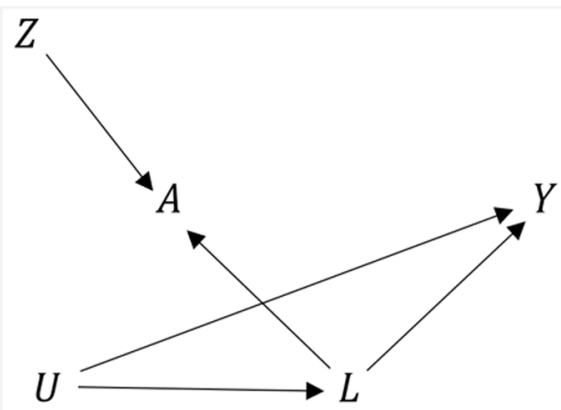

(B) DAG 1

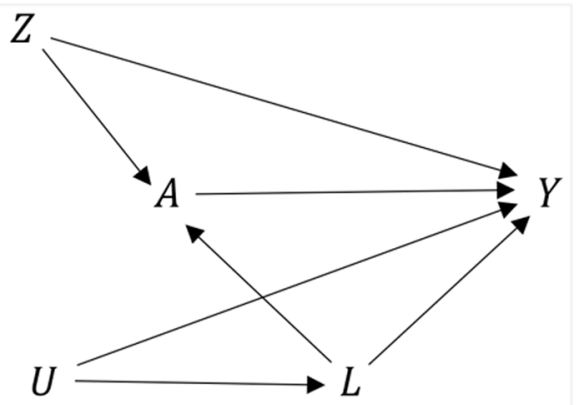

(D) DAG 3

Fig. 1 The causal diagrams representing the simulation mechanisms considered in this study. DAGs 1-3 are subsets of the main DAG and represent the simulation settings $1-3$, respectively. Here, $Z$ is the randomization variable, $A$ is the treatment, $L$ is a vector of measured confounders, $U$ is unmeasured confounders, and $Y$ is the outcome. Abbreviations: DAG: directed acyclic graph

way other than $A$, the exclusion restriction assumption is violated [37].

\section{Target parameter}

The risk difference (RD) was calculated as the target parameter of interest. The odds ratio is a widely used association measure for a binary outcome. However, since the odds ratio is a non-collapsible measure, we chose not to use this measure [38]. Our effect estimates are marginal for some methods and conditional over covariates for other methods. For example, the outcome model for the naive methods, naive and first-stage adjusted 2SLS, and IP-weighted PP do not include any covariates, and the resulting effect estimates are marginal. On the other hand, the baseline-adjusted methods, both-stages adjusted 2SLS and 2SRI, include measured covariates in the outcome model, and the resulting effect estimates are conditional on covariates. Therefore, we chose to use RD, which is a collapsible effect measure that gives equivalent and comparable marginal or conditional estimates [39].

To estimate RD, we can use the binomial regression with an identity link function as the outcome model. However, the binomial model fitting frequently shows convergence issues with adjusting for covariates in the model and requires guessing reasonable starting values which is almost impossible in the simulation settings. Naimi and
Whitcomb [40] suggested to use the generalized linear model (GLM) with a Gaussian (i.e., normal) distribution in such scenarios as an alternative to the binomial regression even if the outcome variable is binary. The authors suggested using the robust sandwich standard error for valid standard errors in this setting. In our study, we followed the GLM method described by Naimi and Whitcomb as the outcome model for all the estimators other than the IP-weighted PP [40]. For the IP-weighted PP estimator, since we have to incorporate IP weights in the outcome model, we used the weighted GLM for the Gaussian family with an identity link function and robust standard error.

\section{Simulation setup}

We followed two established simulation structures described by Young et al. [26], and by Palmer et al. [19] with modifications. Under both structures, we consider point-treatment settings (treatment is administered only once) and each individual is assigned to the treatment $(Z=1)$ or to standard care $(Z=0)$. We considered three data generating mechanisms, where $A$ is the treatment, $Y$ is the binary outcome of interest, $L=\left(L_{1}, L_{2}\right)$ is a vector of measured baseline confounders, and $U$ is unmeasured confounders. We considered one set of variables in the following causal order for all three settings: $Z, U, L_{1}, L_{2}, A, Y$. One such example of a real-world scenario could be 
estimating the effect of the seasonal influenza vaccine on flu in 6 months among adults. The three data-generating mechanisms considered in this study are different than each other in terms of the underlying assumption of measured and unmeasured confounding and exclusion restriction. Figure 1(A) shows the overall directed acyclic graph (DAG) of the data generating process. For simulation settings 1-3 described below, we simulated data from the following algorithms (Eqn. (1)):

$$
\begin{gathered}
Z \sim \operatorname{Bernoulli}(0.5) \\
U \sim f_{U} \\
L_{1} \sim \operatorname{Normal}\left(\lambda_{0}+\lambda_{1} U, \sigma\right) \\
L_{2} \sim \operatorname{Bernoulli}\left(p_{L 2}\right) \\
A \sim \operatorname{Bernoulli}\left(p_{A}\right) \\
Y \sim \operatorname{Bernoulli}\left(p_{Y}\right) .
\end{gathered}
$$

The description of each three generating processes with parameterization is described below in detail.

\section{Setting 1: exclusion restriction satisfied and no unmeasured confounding}

Young et al. [26] described the simulation structure for longitudinal setting, whereas we used a simplified version of this simulation by considering the point treatment setting and having only baseline covariate measurements. Data for setting 1 are generated from our example of the influenza vaccine effect on flu. $Z$ and $A$ are the randomization and influenza vaccine status, receptively. In this example, physical activity $\left(L_{1}\right)$ and illness $\left(L_{2}\right)$ are two measured confounders, and smoking is an unmeasured confounder $(U)$. Less physical activity can cause chronic illness, and thus, an arrow from $L_{1}$ to $L_{2}$ in our example could be justified [41]. Besides, smoking typically reduces the level of physical activity $\left(U \rightarrow L_{1}\right)$ [42], increases illness $\left(U \rightarrow L_{2}\right)$ [43], and increases the risk of flu $(U \rightarrow Y)$ [44]. In addition, people with less physical activity and having chronic illness could have influenza vaccine hesitancy and are less likely to take the vaccine $(L \rightarrow A)$ [45]. DAG 1 in Fig. 1(B) is a subset of Fig. 1(A), that shows the data generating process for setting 1 . The exclusion restriction assumption is satisfied for this simulation framework. To get an unbiased effect of the influenza vaccine on flu, we must block all open backdoor paths between the vaccine and flu. As shown in Fig. 1(B), even though we have an unmeasured confounder (e.g., smoking), adjusting for measured confounders can be sufficient to block the backdoor path between vaccine status and flu status. Whether or not we measure or adjust for smoking does not impact our ability to obtain unbiased treatment effect estimates of the influenza vaccine on flu. In other words, adjusting only for measured confounders using an appropriate method (e.g., IP-weighted PP, 2SLS, 2SRI) should give us an unbiased effect estimate of the vaccine on flu. Hence, this Setting 1 is a fair scenario for both PP and IV-based methods where PP methods that adjust for $L$ and the IV-based methods should all be unbiased.

Using the Eqn. (1), the following are considered to simulate the data for settings $1: f_{U}=\operatorname{Uniform}(0,1), \lambda_{0}=$ $0, \lambda_{1}=6, \sigma=2, p_{L 2}=\operatorname{logit}\left(-5+3 U+1.25 L_{1}\right), p_{A}=$ $\operatorname{logit}\left(\alpha_{0}+\alpha_{1} Z+\alpha_{2} L_{1}+\alpha_{3} L_{2}+\alpha_{4} U\right)$, and $p_{Y}=\operatorname{logit}\left(\theta_{0}+\right.$ $\left.\theta_{1} A+\theta_{2} L_{1}+\theta_{3} L_{2}+\theta_{4} U+\theta_{5} Z\right)$. Here, $\alpha_{0}$ is associated with the nonadherence rate; $\alpha_{1}=0.6$ is the coefficient associated with randomization; $\alpha_{2}=0.4$ and $\alpha_{3}=0.35$ are coefficients associated with the measured covariates; $\alpha_{4}=0$. Also, $\theta_{0}$ is associated with the event rate; $\theta_{1}=0$ is the null treatment effect (and thus no arrow from $A$ to $Y$ in DAG 1$) ; \theta_{2}=0 ; \theta_{3}=0 ; \theta_{4}$ determines the magnitudes of unmeasured confounders, and $\theta_{5}=0$ indicates the exclusion restriction is satisfied in this setting. Under different choices of $\alpha_{0}$, we considered six levels of deviations from adherence in each arm: 10, 20, 40, 60, 80, and 90\%. The parameter choices are summarized in Table 3(A). For each of the nonadherence scenarios, we considered two sets of $\theta_{4}$ values, making a total of 12 scenarios. We set $\theta_{4}$ $=8$ and 0.5 for strong and weak unmeasured confounders, respectively. The full list of parameters considered for all 12 scenarios for setting 1 is shown in Appendix D Table 5.

\section{Setting 2: exclusion restriction satisfied, unmeasured confounding present}

Palmer et al. [19] described the simulation structure for point-treatment settings where the exclusion restriction assumption of the IV assumption is violated. We slightly modified the structure and considered two versions of this simulation: the exclusion restriction of IV assumption is not violated but there exists unmeasured confounding (Fig. 1(C); DAG 2), and the exclusion restriction assumption is violated (Setting 3; described later). Recall our example on estimating the influenza vaccine effect on flu where information on smoking is unmeasured. As shown in DAG 2, smoking status is a barrier to receive the influenza vaccine $(U \rightarrow A)$ [45], but it increases the risk of flu $(U \rightarrow Y)$ [44]. Given that the study is a randomized trial, the exclusion restriction could be satisfied in this setting, but there could be unmeasured confounding. Unlike setting 1, adjusting only for measured confounders (e.g., physical activity and illness) may not be sufficient to get an unbiased effect estimate of the vaccine on flu using the PP methods [14].

Using the Eqn. (1), the following are considered to simulate the data for settings $2: f_{U}=\operatorname{Bernoulli}(0.5), \lambda_{0}=$ $3, \lambda_{1}=0, \sigma=0.5, p_{L 2}=\operatorname{logit}\left(-3.5+0.6 L_{1}\right), p_{A}=$ $\alpha_{0}+\alpha_{1} Z+\alpha_{2} L_{1}+\alpha_{3} L_{2}+\alpha_{4} U$, and $p_{Y}=\theta_{0}+\theta_{1} A+\theta_{2} L_{1}+$ $\theta_{3} L_{2}+\theta_{4} U+\theta_{5} Z$. We considered $\alpha_{1}=0.25, \alpha_{2}=0.02$, $\alpha_{3}=0.04$, and $\alpha_{4}=0.05$. Under different choices of $\alpha_{0}$, we considered six levels of nonadherence: 10, 20, 40, 60, 80 , and $90 \%$. The parameter choices are summarized in 
Table 3 The nonadherence rates per arm for different choices of $\alpha_{0}$ for simulation settings $1-3$ with $\left(\alpha_{1}, \alpha_{2}, \alpha_{3}, \alpha_{4}\right)=(0.6,0.4,0.35,0)$ for setting $1,(0.25,0.02,0.04,0.05)$ for setting 2 , and $(0,0.01,0.04,0)$ for setting 3

\begin{tabular}{|c|c|c|c|c|c|c|c|}
\hline \multirow{2}{*}{ Scenario } & \multirow{2}{*}{ Arm } & \multicolumn{2}{|c|}{ A. Setting 1} & \multicolumn{2}{|c|}{ B. Setting 2} & \multicolumn{2}{|c|}{ C. Setting 3} \\
\hline & & $\alpha_{0}$ & Nonadherence & $\alpha_{0}$ & Nonadherence & $\alpha_{0}$ & Nonadherence \\
\hline \multirow{2}{*}{1} & $Z=1$ & 0.72 & 10 & 0.55 & 11 & 0.86 & 10 \\
\hline & $Z=0$ & -4.06 & 10 & 0.02 & 11 & 0.06 & 10 \\
\hline \multirow{2}{*}{2} & $Z=1$ & -0.23 & 20 & 0.46 & 20 & 0.76 & 20 \\
\hline & $Z=0$ & -3.14 & 20 & 0.12 & 21 & 0.16 & 20 \\
\hline \multirow{2}{*}{3} & $Z=1$ & -1.47 & 40 & 0.25 & 41 & 0.56 & 41 \\
\hline & $Z=0$ & -1.92 & 40 & 0.32 & 41 & 0.36 & 40 \\
\hline \multirow{2}{*}{4} & $Z=1$ & -2.52 & 60 & 0.05 & 61 & 0.36 & 60 \\
\hline & $Z=0$ & -0.85 & 60 & 0.52 & 61 & 0.57 & 60 \\
\hline \multirow{2}{*}{5} & $Z=1$ & -3.76 & 80 & -0.15 & 81 & 0.16 & 80 \\
\hline & $Z=0$ & 0.39 & 80 & 0.70 & 80 & 0.77 & 80 \\
\hline \multirow{2}{*}{6} & $Z=1$ & -4.72 & 90 & -0.25 & 91 & 0.06 & 90 \\
\hline & $Z=0$ & 1.35 & 90 & 0.80 & 89 & 0.86 & 90 \\
\hline
\end{tabular}

Table 3(B). For each of the six nonadherence scenarios, we considered five scenarios of the treatment effect of interest $\left(\theta_{1}\right)$ and two versions of confounding $\left(\theta_{4}\right)$, making a total of 60 scenarios. We set the treatment effect of interest as $\theta_{1}=\{-0.2,-0.05,0,0.05,0.2\}$ and confounding as $\theta_{4}=$ 0.05 and 0.4 respectively for weak and strong confounding. In this setting, the exclusion restriction is satisfied so that $\theta_{5}=0$. The complete list of parameters considered for all 60 scenarios for setting 2 is shown in Appendix D Table 6.

\section{Setting 3: exclusion restriction violated}

If the exclusion restriction is met, $Z$ affects the outcome $Y$ only through the treatment $A$ [36]. For example, if $Z$ represents randomization in a double-blind randomized controlled trial, $Z \rightarrow A \rightarrow Y$ is expected and the exclusion restriction assumption usually met. However, as discussed by Brookhart et al. [37], if the instrument $Z$ represents physician's prescribing preference, there could be a direct effect of $Z$ on $Y$ because physicians tend to prescribe selective drugs based on their experience about safety and efficacy of drugs. Recall our motivating example of exploring the effect of the seasonal influenza vaccine on flu. Let $Z$ be the physician's prescribing preference on whether an individual is recommended the seasonal influenza vaccine. Based on the experience, the physicians could prescribe the vaccine only to subjects with a higher risk of the flu [46]. Therefore, in addition to the influence of $Z$ on $Y$ through $A, Z$ could directly influence $Y$. The data generating mechanism for this setting is shown in Fig. 1(D; DAG 3). Since the exclusion restriction assumption is violated in this setting, the IV methods (e.g., 2SLS and 2SRI) are expected to produce biased estimates no matter whether we adjust for measured confounders (e.g., physical activity and illness) [24].
Using the Eqn. (1), the following are considered to simulate the data for settings 3: $f_{U}=\operatorname{Bernoulli}(0.5), \lambda_{0}=$ $3, \lambda_{1}=0.05, \sigma=0.5, p_{L 2}=\operatorname{logit}\left(-3.5+0.6 L_{1}+\right.$ $0.1 U), p_{A}=\alpha_{0}+\alpha_{1} Z+\alpha_{2} L_{1}+\alpha_{3} L_{1}+\alpha_{4} U$, and $p_{Y}=$ $\theta_{0}+\theta_{1} A+\theta_{2} L_{1}+\theta_{3} L_{2}+\theta_{4} U+\theta_{5} Z$. We considered $\alpha_{1}=0, \alpha_{2}=0.01, \alpha_{3}=0.04$, and $\alpha_{4}=0$. The same as before, there are six levels of nonadherence for different choices of $\alpha_{0}: 10,20,40,60,80$, and 90\%. The parameter choices are summarized in Table $3(\mathrm{C})$. For each nonadherence scenario, we set two versions of $\theta_{1}$ and two versions of $\theta_{5}$, making a total of 24 scenarios. We set $\theta_{1}$ $=0$ for the null treatment effect and 0.2 for the non-null effect. $\theta_{5}$ determines the severity of the exclusion restriction assumption violation. A small $\theta_{5}$ value indicates a minor violation of the exclusion restriction, and a large value indicates a severe violation. We set these $\theta_{5}$ values as 0.05 and 0.2 in this study. The complete list of parameters for these 24 scenarios is shown in Appendix D Table 7.

\section{Simulation \\ Sample size and iterations}

To assess the performance of the estimation methods, we generated 2,000 samples (approximately 1,000 per arm) with 1000 iterations for each scenario based on the above DAGs. R version 4.1.0 was used to perform the analysis.

\section{Performance metrics}

We assessed the performance of the models through several measures, such as bias, standard error (SE), mean squared error (MSE), and 95\% confidence interval (CI) coverage probability. This allows us to compare the performance of different methods in terms of accuracy, precision, and coverage. The following definitions are used to define these measures [47]: 


$$
\begin{gathered}
\mathrm{Bias}=\frac{1}{n_{\text {sim }}} \sum_{i=1}^{n_{\text {sim }}} \hat{\beta}_{i}-\beta=\hat{\bar{\beta}}-\beta \\
\mathrm{SE}=\sqrt{\frac{1}{n_{\text {sim }}-1} \sum_{i=1}^{n_{\text {sim }}}\left(\hat{\beta}_{i}-\hat{\bar{\beta}}\right)^{2}} \\
\mathrm{MSE}=\frac{1}{n_{\text {sim }}} \sum_{i=1}^{n_{\text {sim }}}\left(\hat{\beta}_{i}-\beta\right)^{2} \\
\text { Coverage }=\frac{1}{n_{\text {sim }}} \sum_{i=1}^{n_{\text {sim }}} 1\left(\hat{\beta}_{\text {lower }, i} \leq \beta \leq \hat{\beta}_{\text {upper }, i}\right),
\end{gathered}
$$

where $\beta$ (i.e., RD) is the true treatment effect of interest, $n_{\text {sim }}$ is number of iterations (1000 in our case), $\hat{\beta}_{i}$ is the estimated RD in the $i$ th iteration, and $\hat{\beta}_{\text {lower }, i}$ and $\hat{\beta}_{\text {upper }, i}$ are the lower and upper $95 \%$ bound of the RD in the $i$ th iteration, respectively.

\section{Results}

We presented the results for the baseline-adjusted PP, IPweighted PP, naive 2SLS, both-stages adjusted 2SLS, naive 2SRI, and both-stages adjusted 2SRI methods in the main text, as they are the main focus of the study. The results for all 14 methods described in Table 1 can be found in Appendix C.

\section{Setting 1: exclusion restriction satisfied and no unmeasured confounding \\ Bias}

Figure 2 shows the bias versus an incremental rate of nonadherence using DAG 1 (null treatment effect) for baseline-adjusted PP, IP-weighted PP, naive and bothstages adjusted 2SLS and 2SRI methods. Under the scenario of weak unmeasured confounders, all methods produce small bias. In the presence of strong unmeasured confounders, the 2SLS and 2SRI estimates have nominal biases (range 0 to 0.02). The baseline-adjusted PP and IP-weighted PP estimates are approximately unbiased, but these estimates are biased (the bias is approximately 0.025 ) beyond $80 \%$ of nonadherence. The bias versus nonadherence comparison for all methods considered in this study is shown in Appendix C Fig. 6.

\section{$S E$, MSE, coverage, and bounds of NPCB}

The 2SLS and 2SRI methods produce higher SE than PP methods (Appendix C Fig. 7). The baseline-adjusted PP and IP-weighted PP also produce high SE beyond 40\% nonadherence. Also, the IP-weighted PP performs the worst beyond $60 \%$ nonadherence, producing very high SE (e.g., approximately twice than both-stages adjusted 2SLS and 2SRI methods). The pattern and amount of SE are the same for weak and strong unmeasured confounders scenarios.

Under the null scenario with weak unmeasured confounders, all methods produce small MSE and some share approximately the same line (Appendix C Fig. 8). The naive and both-stages adjusted 2SLS and 2SRI methods have slightly higher MSE compared to the baselineadjusted PP and IP-weighted PP when the nonadherence

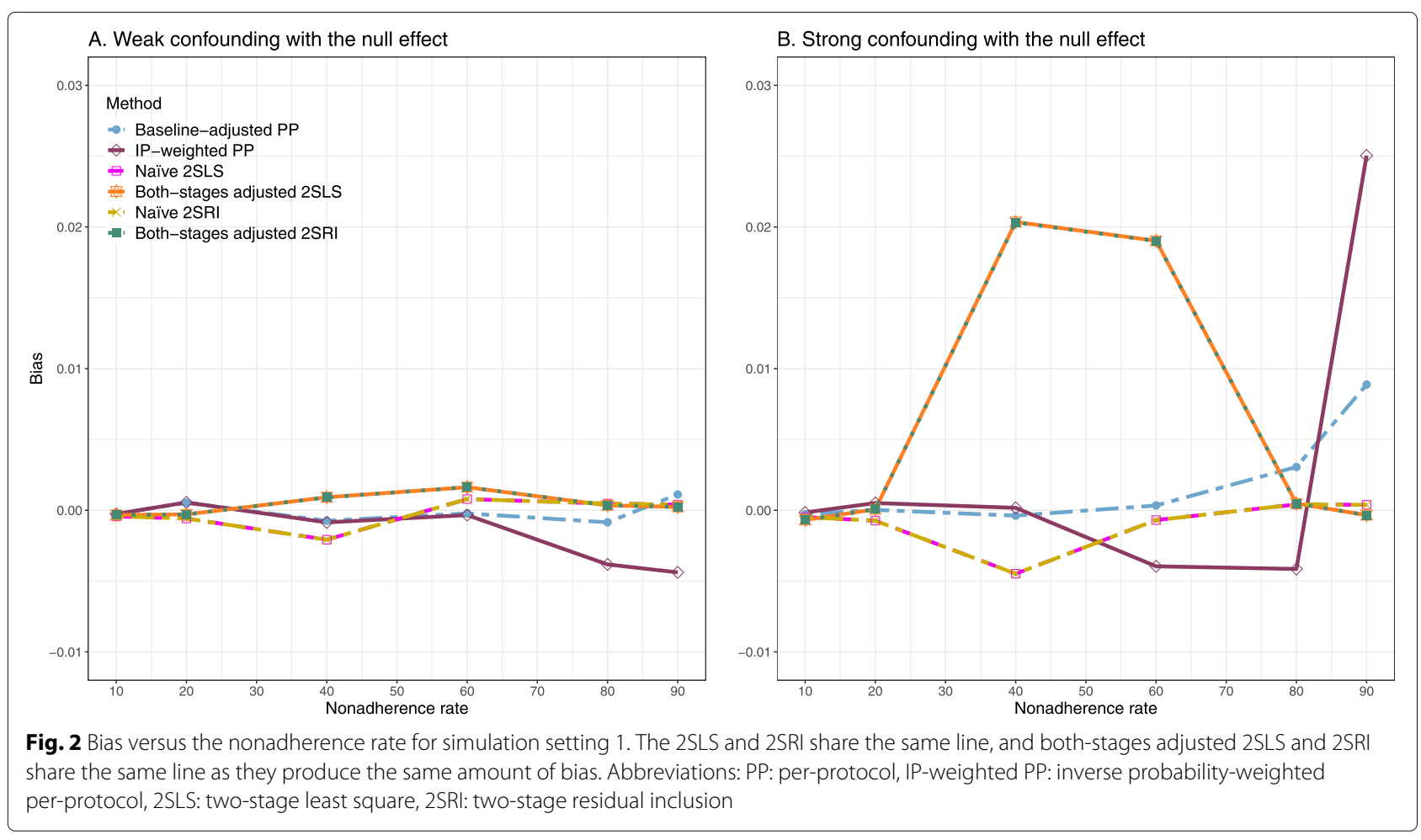


rate is $\leq 60 \%$. For example, MSEs are close to zero for the baseline-adjusted PP and IP-weighted PP methods, but about 0.015 for naive and both-stages adjusted 2SLS and 2SRI methods. On the other hand, the baseline-adjusted PP and IP-weighted PP have more than twice MSE than naive and both-stages adjusted 2SLS and 2SRI methods beyond $60 \%$ nonadherence. Also, the both-stages adjusted 2SLS and 2SRI produce comparatively smaller MSE than the naive 2SLS and 2SRI methods.

The IP-weighted PP produces nominal 95\% coverage for less than $80 \%$ nonadherence, but this method produces low coverage for $\geq 80 \%$ nonadherence (Appendix C Fig. 9). On the other hand, the naive and both-stages adjusted 2SLS and 2SRI produce nominal 95\% coverage regardless of weak or strong unmeasured confounders.

The bounds of the NPCB method using DAG 1 are shown in Appendix C Fig. 10. The NPCB method produces very wide bounds regardless of weak or strong unmeasured confounders. In contrast, the width of bounds is small for $10 \%$ and $90 \%$ nonadherence, and high for $20 \%$ to $80 \%$ nonadherence.

\section{Setting 2: exclusion restriction satisfied, unmeasured confounding present Varying the effect of nonadherence}

We presented bias, SE, MSE, and 95\% coverage probability for different nonadherence rates. We presented the results for a null $(\mathrm{RD}=0)$ and non-null $(\mathrm{RD}=0.2)$ treatment effect scenarios.

Bias Under the scenario of weak unmeasured confounding, all methods produce small bias (Fig. 3). In the presence of strong unmeasured confounding, the baselineadjusted PP and IP-weighted PP produce biased estimates. The amount of bias (range from 0.02 to 0.10 ) is approximately the same for the null and non-null treatment effect scenario. In contrast, the naive 2SLS and 2SRI, and both-stage adjusted 2SLS and 2SRI methods consistently produce unbiased estimates under the null or non-null and weak or strong confounding scenarios. The bias versus nonadherence rate for all methods using DAG 2 is shown in Appendix C Fig. 11.

SE, MSE, coverage, and bounds of NPCB Appendix C Figs. 12 and 13 show the SE and MSE versus nonadherence using DAG 2, respectively. The baseline-adjusted PP and IP-weighted PP produce low coverage for strong confounding scenarios, and these two methods perform poorly beyond 60\% nonadherence (Appendix C Fig. 14). On the other hand, the naive and both-stages adjusted 2SLS and 2SRI consistently have nominal coverage under both null or non-null effects and weak or strong unmeasured confounding scenarios. The NPCB method

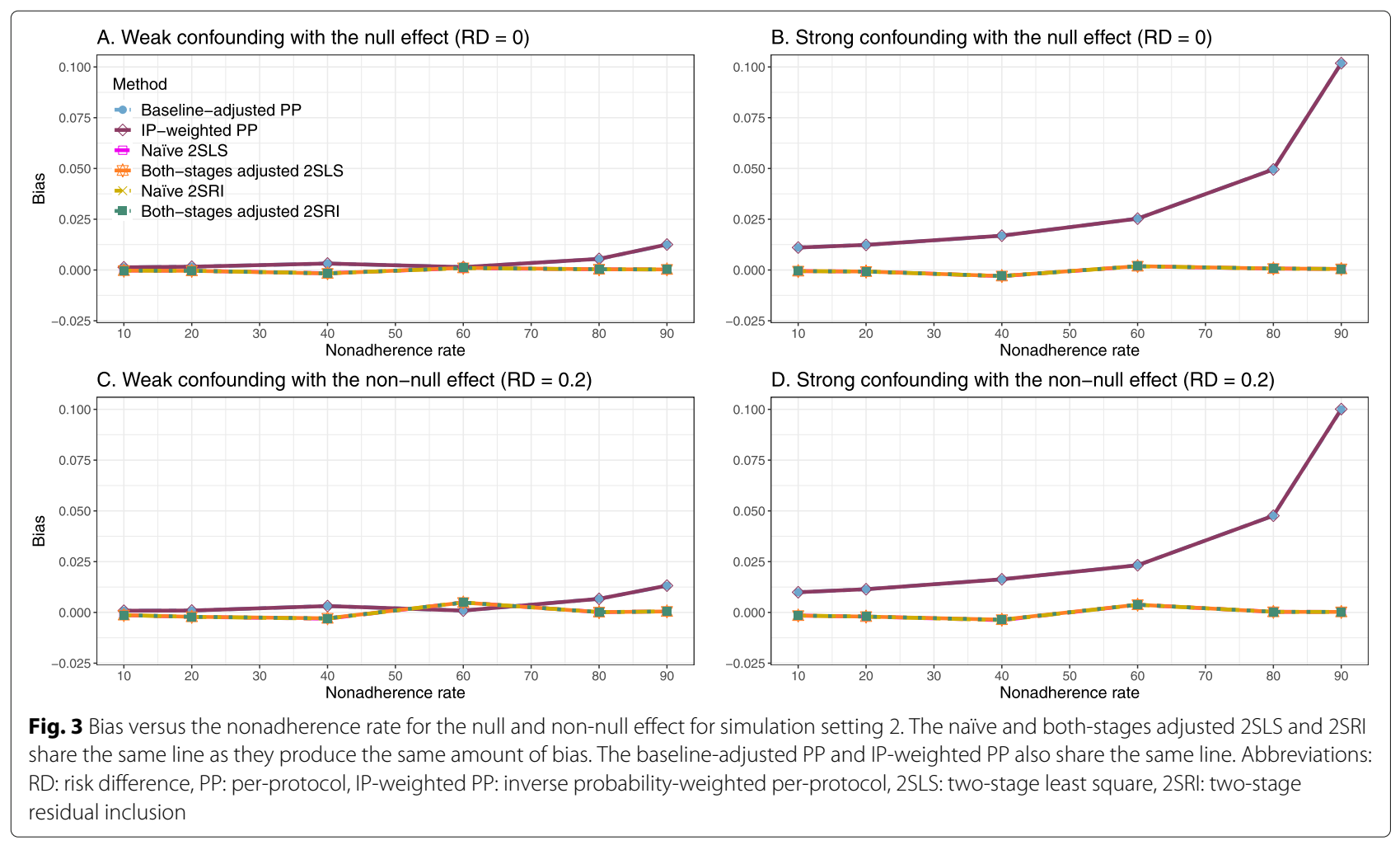


produces a wide bound for both the null and the nonnull effect, regardless of the weak or strong confounding (Appendix C Fig. 15). The width of bounds is very wide for $20 \%$ to $80 \%$ nonadherence.

\section{Varying the effect of the treatment}

We presented bias, SE, MSE, and 95\% coverage probability for different treatment effects. We showed the results for $10 \%$ and $40 \%$ nonadherence rates.

Bias Figure 4 shows the bias versus treatment effect for $10 \%$ and $40 \%$ nonadherence using DAG 2 . All methods produce approximately an unbiased estimate under the weak confounding scenario with $10 \%$ nonadherence. The bias is increased to about 0.015 for baseline-adjusted PP and IP-weighted PP methods under the strong confounding scenario. The bias is more pronounced (about 0.02) for $40 \%$ nonadherence. However, the naive and both-stages adjusted 2SLS and 2SRI methods produce approximately unbiased estimates for any treatment effects under weak or strong confounding. The bias versus treatment effect for all methods using DAG 2 is shown in Appendix C Fig. 16.

SE, MSE, and coverage SE and MSE versus treatment effect for $10 \%$ and $40 \%$ nonadherence using DAG 2 are presented in Appendix C Figs. 17 and 18. The baselineadjusted PP and IP-weighted PP methods produce small coverage under the strong unmeasured confounding scenario (Fig. 19). On the other hand, the naive and bothstages adjusted 2SLS and 2SRI consistently have nominal coverage regardless of the treatment effect and weak or strong unmeasured confounding.

\section{Setting 3: exclusion restriction assumption violated Bias}

Figure 5 shows the bias versus nonadherence rate using DAG 3. We observed that the 2SLS and 2SRI methods produce high biases when the exclusion restriction assumption is violated. The biases are between -0.3 and 0.3 for a minor violation of the exclusion restriction but beyond the range of $[-0.3,0.3]$ when the violation is severe. The baseline-adjusted PP and IP-weighted PP estimates are also biased for minor or severe violations of the exclusion restriction. However, the bias is slightly smaller (range 0.05 to 0.25 ) compared to 2SLS and 2SRI methods. The bias versus nonadherence rate for all methods using DAG 3 is shown in Appendix C Fig. 20.

\section{$S E$, MSE, coverage, and bounds of NPCB}

The SE and MSE versus nonadherence can be found in Appendix C Figs. 21 and 22. In terms of 95\% coverage probability, both PP and IV methods produce low coverage for either minor or severe violations of the exclusion restriction assumption (Appendix C Fig. 23). The NPCB

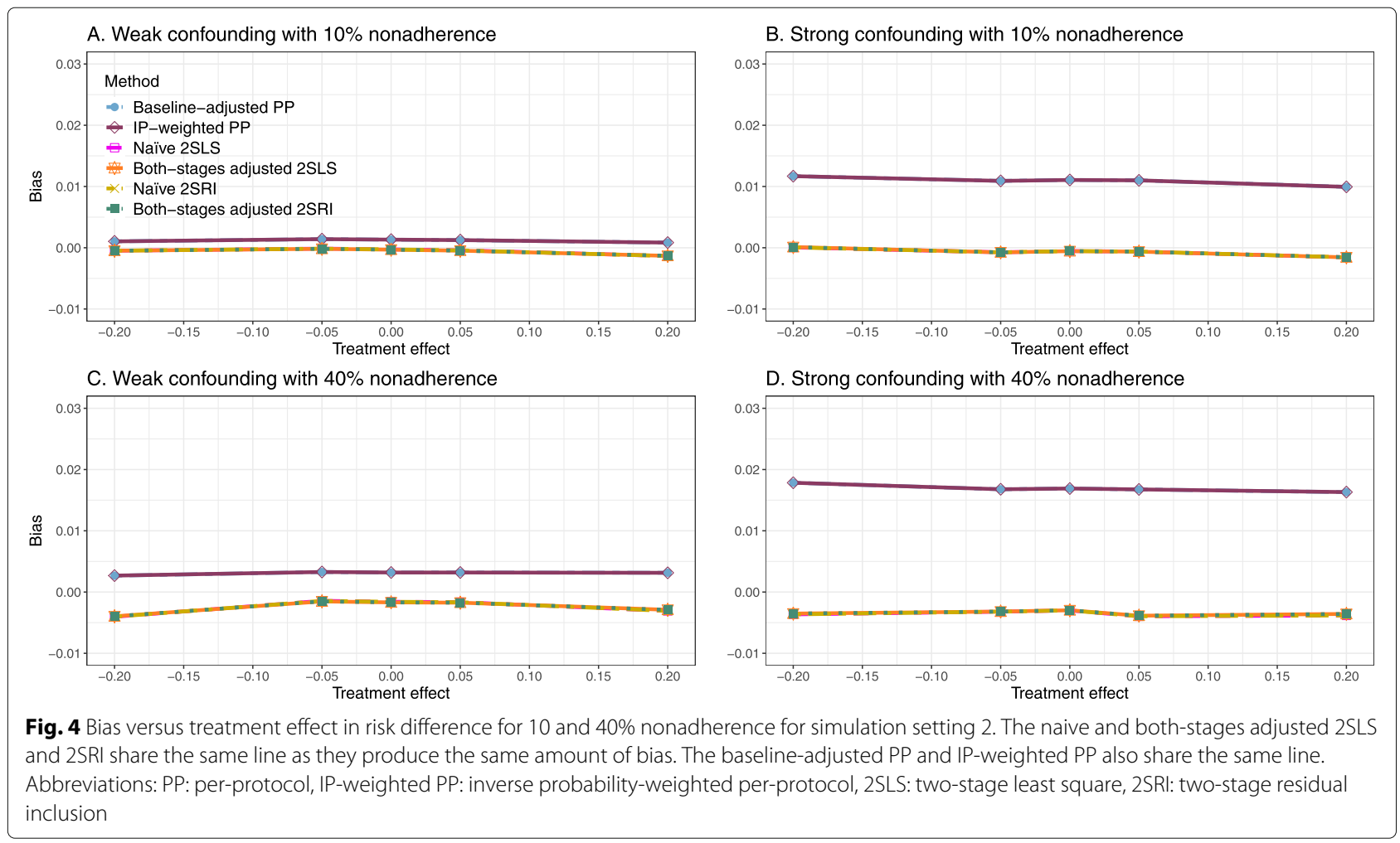




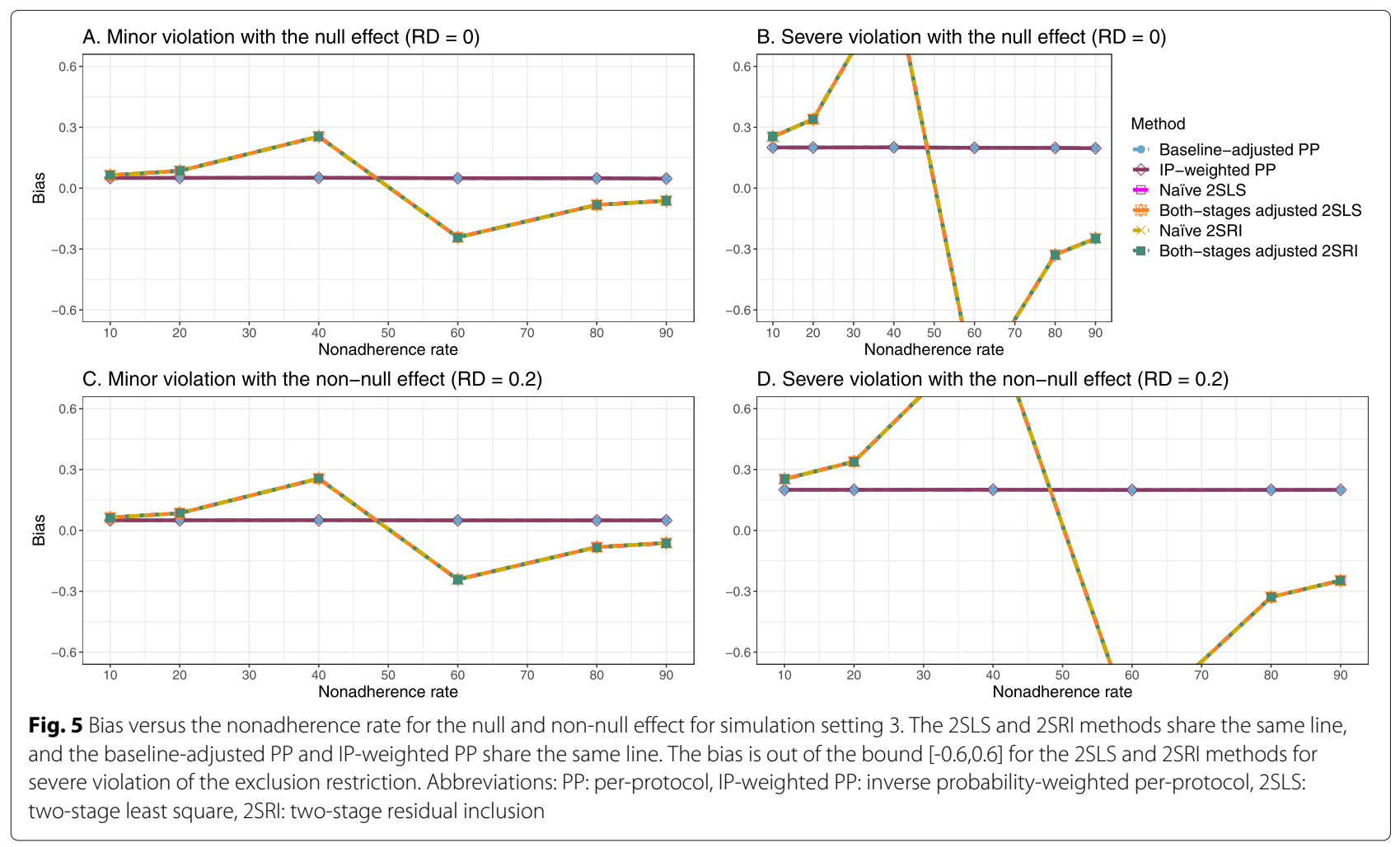

produces wide bounds for all scenarios, regardless of the minor or severe violation of the exclusion restriction assumption (Appendix C Fig. 24).

\section{Sensitivity analysis}

\section{Exclusion restriction satisfied, unmeasured confounding} present

We produced results for a simplified version of DAG 2 to mimic the possible set of variables as in our case study. The DAG is shown in Appendix C Fig. 25(A) and details are described in Appendix $B$. The results are shown in Appendix C Figs. 26-29. As expected, the ITT, PP, and AT methods have biased estimates, while the 2SLS and 2SRI methods produce unbiased estimates.

\section{Exclusion restriction violated}

We produced results when the exclusion restriction assumption is violated, where the setting mimics the possible set of variables as in our case study. The DAG is shown in Appendix C Fig. 25(B) and details are described in Appendix B. The results for bias versus nonadherence are shown in Appendix C Fig. 30. We observed that the 2SLS and 2SRI methods perform worse than even naive methods, no matter whether there is a minor or severe violation of the exclusion restriction assumption.

\section{Sensitivity analyses with a smaller sample size}

We also simulated data for simulation settings $1-3$ by considering 500 samples (approximately 250 per arm). The results are shown in Appendix C Figs. 31-45. The patterns of bias remain the same for all scenarios. As expected, the SEs are higher for the scenarios with 500 samples than those with 2000 samples. Consequently, the MSEs are higher for the scenarios with 500 samples than those with 2000 samples. Also, the IP-weighted PP estimates suffer from non-convergence issues when the nonadherence rate is very high (e.g., 90\%). Overall, the conclusions remain the same for the scenarios with a smaller sample size (500) compared to those from a relatively larger sample size (2000). We further attempted generating data by considering 100 samples (approximately 50 per arm), but faced non-convergence issues due to the low sample size, impacting the stability of the estimates; and hence the results are not shown here.

We have added a flow chart showing the present study's recommendations in Appendix C Fig. 46.

\section{Case study: vitamin A supplementation on childhood mortality}

We used a dataset from a two-arm randomized control trial with 450 villages in northern Sumatra, exploring 
the effect of vitamin A supplementation on childhood (1-year) mortality $[48,49]$. The villages were randomized to either receive vitamin A supplementation or act as a control group for a year. A total of 23,682 children aged 12-71 months from these villages received a large oral dose of vitamin A supplementation ( $\mathrm{n}=$ $12,094)$ or no treatment ( $\mathrm{n}=11,588)$. Not every child took the assigned vitamin A supplementation in the treatment group, and the nonadherence rate was $20 \%$ in the group receiving vitamin A supplements. Vitamin A supplements were not available to children in the control group. This was a randomized trial, and extraneous factors should not influence the instrument $Z$ (the randomization variable) [48]. Therefore, there should not be any direct arrow from $Z$ to $Y$, or $Z$ to $Y$ through another pathway other than $A$, meaning that the exclusion restriction assumption for this trial should be reasonably met. Previous studies reported very wide bounds from the same dataset when analyzed by the NPCB method [19, $20]$. In the present study, we also assessed the performances of two other IV methods (2SLS and 2SRI) in the same data. Since there was no measured confounder in the dataset, we did not apply baseline covariate-adjusted methods. Instead, we reported the results for the ITT, naive $P P$, naïve $A T$, naïve $2 S L S$, naïve $2 S R I$, and $\mathrm{NPCB}$ methods.

The event rate was 5.1 per 1,000 . Table 4 shows the risk difference per 1,000 in the treatment group than the control, standard error, and associated 95\% CI. According to the ITT estimate, 2.6 fewer deaths (95\% CI: 0.8-4.4) were associated with vitamin A supplementation. However, the ITT estimate do not take into account that a large proportion of children failed to receive vitamin A as prescribed. The PP and AT analyses estimate how efficacious was vitamin A supplementation among those children who actually took the treatment. According to the naive PP, approximately 5.2 less deaths (95\% CI: 3.5-6.8) were associated with vitamin A supplementation among those children who actually took the treatment, while it was 6.5 fewer deaths (95\% CI: 4.9-8.1) as per naive AT. The 2SLS and 2SRI produce identical results; approximately 3.2 less deaths (95\% CI: 1.0-5.5) among the compliers. In contrast, the SE was slightly higher for 2SLS and 2SRI compared to other methods. The bound for the NPCB was reported to be very wide (-5.4 to 194.6) $[19,20]$.

\section{Discussion}

\section{Summary of the findings}

We used three data-generating mechanisms to study the performance of various statistical methods to deal with nonadherence in pragmatic trial settings with a binary point treatment and a binary outcome. We considered three settings such as (1) exclusion restriction is satisfied and there is no unmeasured confounding; (2) the exclusion restriction assumption is satisfied but there is unmeasured confounding present; (3) the exclusion restriction assumption is violated. No single method is the best in all situations. For setting 1, it is expected that baseline adjusted and IP-weighted PP, and 2SLS and 2SRI estimates are unbiased. We observed that the naive and both-stages adjusted 2SLS and 2SRI methods perform very well in terms of bias and coverage for any nonadherence rate. The baseline-adjusted PP and IP-weighted PP methods outperform the 2SLS and 2SRI methods for below a certain nonadherence rate but show very high bias beyond that point. For our simulation settings, $80 \%$ was that cut-point. As expected, the baseline-adjusted PP and IP-weighted PP also have high SE beyond that point. For setting 2, the baseline-adjusted PP and IP-weighted PP perform well in terms of bias, SE, and coverage compared to the 2SLS and 2SRI methods when there is weak unmeasured confounding. However, these PP methods perform poorly under the strong unmeasured confounding. Only naive 2 SLS and 2SRI methods and both-stages adjusted 2SLS and 2SRI methods produce approximately unbiased estimates irrespective of weak or strong unmeasured confounding. As expected, these IV methods have high SEs in almost all

Table 4 Estimated effect of vitamin A supplementation on childhood mortality in a two-arm randomized control trial with 450 villages in northern Sumatra

\begin{tabular}{llll}
\hline Method & RD & SE & $\mathbf{9 5 \% ~ C l ~}$ \\
\hline ITT & -2.58 & 0.93 & $-4.40,-0.76$ \\
Naïve PP & -5.15 & 0.82 & $-6.76,-3.53$ \\
Naïve AT & -6.47 & 0.82 & $-8.08,-4.86$ \\
Naïve 2SLS & -3.23 & 1.16 & $-5.50,-0.95$ \\
Naïve 2SRI & -3.23 & 1.16 & $-5.50,-0.96$ \\
NPCB ${ }^{1}$ & - & - & $-5.39,194.62$ \\
\hline
\end{tabular}

Abbreviations: RD: risk difference per 1,000 in the treatment group than the control; SE: standard error, Cl: confidence interval; ITT: intention-to-treat; PP: per-protocol; AT: as-treated; 2SLS: two-stage least square; 2SRI: two-stage residual inclusion; NPCB: nonparametric causal bound:

${ }^{1}$ The interval estimate from the NPCB is the bound for the average causal estimate per 1,000 than a $95 \% \mathrm{Cl}$. 
scenarios. For setting 3, all methods (PP and IV) perform poorly in terms of bias, SE, MSE, and coverage. As expected, the 2SLS and 2SRI methods perform poorly due to the exclusion restriction assumption violation. On the other hand, the baseline-adjusted PP and IP-weighted PP methods also suffer in terms of bias and coverage, but for a different reason: the instrument acts as an unadjusted confounder so that there exists an open backdoor path between the treatment variable and the outcome. For all situations, the first stage adjusted 2SLS and 2SRI have high biases and small coverage probabilities. The NPCB method produces very wide bounds in almost all scenarios, including not capturing the true value in the presence of a severe violation of the exclusion restriction assumption.

\section{Context in the literature}

It is expected from the theory that the ITT, naive PP, and naive AT methods usually produce biased estimates when there is nonadherence $[14,50-52]$. The NICE guideline also recommended avoiding these methods in the presence of treatment nonadherence [53].

The adjusted PP methods offer consistently excellent performance under the scenarios without unmeasured confounding [14]. However, it is expected from the literature that the baseline adjusted PP and IP-weighted PP methods produce biased estimates when there is unmeasured confounding [14]. Notably, the presence of unmeasured confounding means the violation of the exchangeability assumption [54]. Using the second definition of the PP methods (i.e., excluding those patients entirely from the analysis), we observed that the adjusted PP methods, such as baseline adjusted PP and IP-weighted PP, have high biases in the presence of strong unmeasured confounding. Therefore, we should not use these methods when it is believed that there is strong unmeasured confounding. On the other hand, the IV-based methods can be used to get the unbiased treatment effect estimate even if some of the necessary confounders are unmeasured $[15,55]$. However, the IV methods typically produce a higher SE, which is in-line with the theory [14]. Since the first-stage of the 2SLS and 2SRI is used to predict the treatment, researchers may include covariates only in the first-stage but not in the second stage [28, 29]. Our study showed that adding the covariate only in the first stage of the model but not in the second stage leads to bias estimates. Also, covariates adjustment in IV methods could reduce the $\mathrm{SE}$, which is consistent with the theory [14]. Therefore, we recommend against using first-stage adjusted 2SLS and 2SRI methods, and prefer both-stages adjusted 2SLS or 2SRI methods.

The newer IV-based method, the NPCB, is used by many researchers with the understanding that it can pro- duce a more precise causal bound [20]. The causal bound is a different metric compared to a $95 \%$ confidence interval, and hence coverage cannot be calculated directly. Our simulations, however, showed that this method included the true parameter $100 \%$ of the time in almost all scenarios, except for a severe violation of the exclusion restriction assumption. Although high SE in 2SLS and 2SRI methods motivate using the NPCB method, this method does not adjust for confounders directly and often produces wide bounds [18-20].

It is also expected from the theory that the IV-methods perform poorly when the key assumption of an IV, such as the exclusion restriction, is violated [24]. The exclusion restriction is untestable using the observed data. Usually, the randomization variable in a trial is an ideal instrument. But a weak instrument can lead to biased and imprecise estimates [56, 57]. Therefore, the 2SLS and 2SRI methods should only be used when this IV assumption is plausible. Subject-matter knowledge should be applied to rule out the possibility of violation of the IV assumption. Besides, the no unmeasured confounding assumption is also untestable from the observed data [58]. As expected, the baseline-adjusted PP and IPweighted PP perform poorly and producing high bias when there is unmeasured confounding [14]. However, these PP methods outperform the 2SLS and 2SRI methods in terms of bias, MSE, and coverage if there is no or weak unmeasured confounding. Therefore, we suggest collecting information on covariates, when possible, that could influence the treatment nonadherence to rule out the possibility of having strong unmeasured confounding. Based on empirical observations, previous studies proposed using the IV-methods in the presence of unmeasured confounding, while IP-weighted PP can be used to address nonadherence with no unmeasured confounding $[4,59]$. In the current work, we applied both of these types of methods under a series of data generating mechanisms and compared the results. We additionally suggest using the both-stages adjusted 2SLS or 2SRI, but not the baseline-adjusted PP or IP-weighted PP method when nonadherence is very high (e.g., $\geq 80 \%$ in our case). The IV-based methods can also be used as complementary analysis because the assumptions of these IV methods are different from the non-IV-based methods. If the primary and the complementary analyses result in a similar conclusion, we can have more confidence in the overall conclusion.

\section{Case study}

Using the ITT analysis, 2.6 fewer deaths per 1,000 were associated with vitamin A supplementation was previously reported in the literature [20]. The naive PP and naive AT found 5.2 and 6.5 fewer deaths among those 
children who actually took the treatment than the control group, respectively. Both naive 2SLS and 2SRI found fewer deaths (RD 3.2) among the compliers. The high estimate by the naive AT is not surprising as this method mostly gives a biased treatment effect when we have treatment nonadherence. The PP estimate is relatively different from the results from 2 SLS and 2SRI, might be indicative of the presence of unmeasured confounding. Our second simulation setting shows that the PP methods have a high bias when there is strong unmeasured confounding, but the 2SLS and 2SRI methods still perform very well. Consistent with the literature $[19,20]$, NPCB method produces a very wide bound, which might be due to the high nonadherence rate, as we observed in our simulation.

\section{Strengths}

The present study has several strengths. We considered three comprehensive simulation settings to explore the performance of different methods to deal with treatment nonadherence. We considered both the null and non-null treatment effect scenarios and low to high nonadherence (e.g., $10 \%$ to $90 \%$ ), which enabled us to compare the methods rigorously. For more than $90 \%$ nonadherence, however, most of the algorithms do not converge or produce a very high effect estimate. Moreover, we considered an approximately equal nonadherence rate per arm so that the result has not influenced by the unequal nonadherence rate. We also considered a collapsible effect measure to estimate the treatment effect to ensure that the marginal and conditional treatment effects for any level of the measured confounders are equal and comparable [39]. Utility of our simulation study is that this pedagogic work confirmed theoretical results and helped make the theoretical ideas more accessible to the practitioners and applied researchers. Additionally, we observed similar findings from sensitivity analyses for unmeasured confounding, violation of the exclusion restriction assumption, and small sample size, meaning that our study results are robust.

\section{Limitations and future direction}

Despite its strengths, the study has a few limitations. First, to clearly identify the effect of each factor, we started from a simplistic scenario, but this is easily extendable. Future studies can consider more complex DAGs and add more confounders with both linear and non-linear forms. Second, we did not consider any loss to follow-up, while the differential loss to follow-up can bias the results [5]. The oversimplified point treatment settings and no loss to follow-up also limit guiding about decisions about biasvariance trade-off. Third, our simulation settings have equal nonadherence rates per arm versus one-sided differential nonadherence in our case study. The pattern of bias, SE, MSE, and coverage of all methods under equal versus differential nonadherence are expected to be in the same direction. Our team is exploring this issue in more detail to verify whether differential nonadherence could lead to different conclusions. Fourth, the results in this paper may not be generalizable to other types of outcomes. Some previous studies compared different methods to address treatment nonadherence when the outcome is continuous [60, 61] and time-to-event [26, 62-64]. However, these studies mostly focused on comparing either IV or PP methods with the naive methods, or considered different data structures or treatment strategies (e.g., sustained treatment strategies in longitudinal studies) [26, 64]. Future studies should explore the performance of both IV and PP methods in the point-treatment setting for continuous and time-to-event outcomes, multiple treatment arms, and when effect modifiers are present. In addition to the 2SLS, 2SRI, and NPCB methods, some other IV methods can be used in the same setting to deal with treatment nonadherence. For example, the two-stage predictor substitution (2SPS) can handle non-linearity in the first stage of the 2SLS model and the three-stage least squares method (3SLS) methods can correct the correlation between error terms in the first and second stages of the 2SLS models $[65,66]$. The IV-based structural mean model (SMM) is another method that can address treatment nonadherence [67]. This semi-parametric method uses g-estimation for identification and estimation of the treatment effect after addressing the nonadherence issue. Future studies can explore the performance of these IV methods in contrast to the other IV methods and non-IV methods when dealing with the treatment nonadherence in pragmatic trials.

It is also worth mentioning that the 2SRI estimates can be biased even without violating the exclusion restriction assumption [65], and the level of bias may increase as the severity of confounding increases. Cai et al. [65] reported analytic estimates of such bias in terms of log-odds ratios for a variety of situations. In our study, we observed minimal or no increase in bias as the confounding severity increases. This observation might be due to the fact that we were using RD as effect measure, and the impact was less noticeable compared to that when effect measure was odds ratio. Further studies could assess the bias in the 2SRI method when the effect measure is collapsible.

We require the positivity assumption, i.e., non-zero probability of being exposed or unexposed at every combination of the values of the observed confounders [68]. For randomized trials (e.g., as our case study), that probability of being exposed or unexposed is usually known, and so is belonging to one particular confounder category [68]. However, theoretical violations of positivity may arise when patients with certain characteristics may be ineligible to receive a treatment (e.g., absolute contraindication for a given treatment) [69]. If this is the case, the 
weights from the IP-weighted PP methods can be infinite or very large, and the estimates can be biased and unstable [69]. The high nonadherence rate or small sample size may lead to near positivity violations, which is also responsible for large IP weights. Therefore, even when there is no unmeasured confounding but high nonadherence rates, the biased and large SE in our simulation results could be due to the near positivity violations. Methods such as truncation and overlap weights have been proposed in the literature to deal with the large IP weight problem [70, 71]. On the other hand, a modified causal estimator has been proposed to deal with the positivity issue in the IV estimation [72, 73].

When the treatment is sustained but time-varying, the same methods that we considered in this study are not generally adequate, and methods have been extended to address those scenarios [26, 27]. Naïve methods such as ITT, naïve PP, or naïve AT ignore time-varying confounding and can produce biased estimates [9]. There exists some new literature exploring nonadherence in a sustained treatment strategy when longitudinal postbaseline prognostic variable measures are available, which is beyond the scope of our point-treatment study.

\section{Conclusion}

Besides the research question, the choice of the methods should come from researcher's understanding of the underlying data generating mechanism and available data (e.g., extent of unmeasured confounding and a potential violation of exclusion restriction). An additional consideration includes the nonadherence rate (e.g., high vs. low). Under nearly an ideal situation where there are unmeasured confounders but adjusting for measured confounders can give an unbiased effect estimate, the naive and both-stages adjusted but not first-stage adjusted 2SLS and 2SRI methods perform very well in terms of bias and coverage for any nonadherence rate. Although the naive and both-stages adjusted 2SLS and 2SRI methods give almost identical bias, both-stages adjusted 2SLS and 2SRI methods improve the precision and reduce overall MSE. In the same setting, the baseline-adjusted PP and IP-weighted PP outperform these 2SLS and 2SRI methods in terms of bias, SE, and MSE for $<80 \%$ nonadherence, but these PP methods show very high bias and MSE for $\geq 80 \%$ nonadherence rate. If there is no unmeasured confounding, the baseline-adjusted PP and IP-weighted PP consistently outperform the 2SLS and 2SRI methods in terms of bias, SE, MSE, and coverage. However, these two PP methods perform poorly when some necessary confounders are unmeasured and measured confounders cannot sufficiently block the backdoor paths between the treatment and the outcome. On the other hand, the 2SLS and 2SRI produce high biases and low coverage probabilities when there exists violation of the exclusion restriction assumption. The baseline-adjusted PP and IP-weighted PP can also have biased estimates when the exclusion restriction is violated. However, the baseline-adjusted PP and IP-weighted PP can produce unbiased estimates if all open backdoor paths between the treatment variable and the outcome can be blocked so that the association between the instrument and outcome is nullified. Therefore, when possible, we recommend collecting information on necessary covariates that predict adherence and addressing them appropriately in the analyses. Collecting information on those covariates or augmentation of external data sources from electronic health records could reduce the impact of having strong unmeasured confounding. Since assumptions of PP methods and IV methods are different and often untestable, we suggest analyzing the data using both PP methods (baseline-adjusted PP or IP-weighted PP) and IV-methods (both-stages adjusted 2SLS or 2SRI) and reporting both results. Analysts are more likely to come up with a robust conclusion of the real-world effect of a treatment if they have similar findings from different analyses requiring different assumptions.

\section{Appendix A}

This section describes the estimation methods considered in this study and the assumptions of these estimation methods.

\section{Description of the estimation methods}

Let $Z$ is the two-arm randomization variable, $A$ is the binary treatment variable, $L=\left(L_{1}, L_{2}\right)$ is a vector of measured confounders, and $Y$ is the binary outcome. The risk difference (RD) is our target parameter of interest. The binomial model with an identity link function can be used to estimate RD. Since the binomial model fitting frequently shows convergence issues with adjusting for covariates in the model, the Poisson or Gaussian regression with an identity link function and robust sandwich standard error can be used as an alternative [40]. In the present study, we considered intention-to-treat (ITT), naive per-protocol (PP), naive as-treated (AT), baseline adjusted ITT, baseline adjusted PP, IP-weighted PP, twostage least square (2SLS), two-stage residual inclusion (2SRI), and non-parametric causal bound (NPCB) methods to estimate RD and associated parameters for a twoarm pragmatic trial. The description of calculating RD using these methods is given below.

\section{ITT}

The ITT models $Z$ on $Y$ without adjustment for $L$. The model can be written as

$$
\operatorname{Pr}(Y=1)=\beta_{0}+\beta_{\text {ITT }} Z .
$$

Then $\hat{\beta}_{\text {ITT }}$ is the maximum likelihood estimate (MLE) of $\beta_{\text {ITT }}$ and is the estimated RD. 


\section{Naive PP}

The naive PP models $Z$ on $Y$ among those subjects who receive the treatment according to the protocol $(Z=A)$ but without adjustment for $L$. The model can be written as:

$$
\operatorname{Pr}(Y=1)=\beta_{0}+\beta_{\text {Naive-PP }} Z \text { for } \mathrm{Z}=\mathrm{A} .
$$

Then $\hat{\beta}_{\text {Naive-PP }}$ is the estimated RD.

\section{Naive AT}

The naive AT models $A$ on $Y$, but does not consider whether individuals randomized to the treatment groups. The model cannot adjust for $L$ and can be written as:

$$
\operatorname{Pr}(Y=1)=\beta_{0}+\beta_{\text {Naive-AT }} A .
$$

Then $\hat{\beta}_{\text {Naive-AT }}$ is the estimated RD.

\section{Baseline-adjusted ITT}

The baseline-adjusted ITT is the same as ITT but it adjusts for $L$ :

$$
\operatorname{Pr}(Y=1)=\beta_{0}+\beta_{\mathrm{B}-\mathrm{ITT}} Z+\beta_{2} L_{1}+\beta_{3} L_{2} .
$$

Then $\hat{\beta}_{\mathrm{B}-\mathrm{ITT}}$ is the estimated RD.

\section{Baseline-adjusted PP}

The baseline-adjusted PP is the same as naive PP but it adjusts for $L$ :

$$
\operatorname{Pr}(Y=1)=\beta_{0}+\beta_{\mathrm{B}-\mathrm{PP}} Z+\beta_{2} L_{1}+\beta_{3} L_{2} \quad \text { for } \mathrm{Z}=\mathrm{A} .
$$

Then $\hat{\beta}_{\mathrm{B}-\mathrm{PP}}$ is the estimated RD.

\section{IP-weighted PP}

The method creates inverse probability adherence weights to generate a pseudo population to estimate the treatment effect by removing the effect of nonadherence [25]. We used a logistic regression to estimate the adherence probabilities among those subjects who receive the treatment according to the protocol $(Z=A)$ as follows

$$
\operatorname{logit}(\operatorname{Pr}(A=1))=\gamma_{0}+\gamma_{1} L_{1}+\gamma_{2} L_{2} .
$$

The predicted probability from the above model is the probability of adherence $P_{A}$. We calculated the stabilized weights to prevent from extreme weights $[7,30]$. The stabilized inverse probability of adherence weights can be calculated as

$$
\mathrm{W}_{\text {stabilized }}=\operatorname{Pr}(A=1) \frac{A}{P_{A}}+\operatorname{Pr}(A=0) \frac{1-A}{1-P_{A}} .
$$

To estimate the RD, the weighted outcome model is used, which can be written as

$$
\operatorname{Pr}(Y=1)=\beta_{0}+\beta_{\text {IPW-PP }} Z \quad \text { with weight }=\mathrm{W}_{\text {stabilized }} .
$$

Then $\hat{\beta}_{\text {IPW-PP }}$ is the estimated RD.

\section{Naïve 2SLS}

There are two stages in the 2SLS method. The instrument $(Z)$ is modelled to $A$ in the first stage, and then the predicted treatment is modelled to $Y$ in the second stage [31]. There is no adjustment for $L$ in either stage of the model. We used a logistic regression in the first stage of the model, which can be written as

$$
\operatorname{logit}(\operatorname{Pr}(A=1))=\gamma_{0}+\gamma_{1} Z \text {. }
$$

Then the second stage model can be written as follows

$$
\operatorname{Pr}(Y=1)=\beta_{0}+\beta_{\text {Naive-2SLS }} \widehat{A},
$$

where $\hat{A}$ is the predicted treatment from the first stage, and then $\hat{\beta}_{\text {Naive-2SLS }}$ is the estimated RD.

\section{First-stage adjusted 2SLS}

The first-stage adjusted 2SLS is the same as naive 2SLS except it adjusts for $L$ in the first stage of the model [28, 29 ]. The same as naive 2 SLS, we used a logistic regression in the first stage of the model

$$
\operatorname{logit}(\operatorname{Pr}(A=1))=\gamma_{0}+\gamma_{1} Z+\gamma_{2} L_{1}+\gamma_{3} L_{2} .
$$

Then the second stage model can be written as follows

$$
\operatorname{Pr}(Y=1)=\beta_{0}+\beta_{1 \text { Stage-2SLS } \widehat{A}}
$$

where $\hat{A}$ is the predicted probabilities for $A$ from the first stage, and then $\hat{\beta}_{1 \text { Stage-2SLS }}$ is the estimated RD.

\section{Both-stages adjusted 2SLS}

The both-stages adjusted 2SLS is the same as naive 2SLS except it adjusts for $L$ in both stages of the model. The same as naive and first-stage adjusted 2SLS, we used a logistic regression in the first stage of the model

$$
\operatorname{logit}(\operatorname{Pr}(A=1))=\gamma_{0}+\gamma_{1} Z+\gamma_{2} L_{1}+\gamma_{3} L_{2} .
$$

Then the second stage model can be written as follows

$$
\operatorname{Pr}(Y=1)=\beta_{0}+\beta_{\text {BStage-2SLS }} \widehat{A}+\beta_{2} L_{1}+\beta_{3} L_{2},
$$

where $\hat{A}$ is the predicted probabilities for $A$ from the first stage, and then $\hat{\beta}_{\mathrm{BStage}}$-2SLS is the estimated RD.

\section{Naïve 2SRI}

Similar to the 2SLS, there are two stages in the 2SRI method. But the instrument $(Z)$ is modelled to $A$ in the first stage, and then the residuals from the first stage and $A$ are modelled to $Y$ in the second stage [22]. There was no adjustment for $L$ in either stage of the model. We used a logistic regression in the first stage of the model, which can be written as

$$
\operatorname{logit}(\operatorname{Pr}(A=1))=\gamma_{0}+\gamma_{1} Z \text {. }
$$

The residuals from the first stage can be extracted as follows:

$$
r=A-\widehat{A}
$$


where $\hat{A}$ is the predicted probability for $A$ from the first stage. Then the second stage model can be written as follows

$$
\operatorname{Pr}(Y=1)=\beta_{0}+\beta_{\text {Naive-2SRI }} A+\beta_{2} r
$$

and then $\hat{\beta}_{\text {Naive-2SRI }}$ is the estimated RD.

\section{First-stage adjusted 2SRI}

The first-stage adjusted 2SRI is the same as naive 2SRI except it adjusts for $L$ in the first stage of the model [29]. The same as naive 2SRI, we used a logistic regression in the first stage of the model

$$
\operatorname{logit}(\operatorname{Pr}(A=1))=\gamma_{0}+\gamma_{1} Z+\gamma_{2} L_{1}+\gamma_{3} L_{2} .
$$

The same as before, the residuals from the first stage can be extracted as follows:

$$
r=A-\widehat{A},
$$

where $\hat{A}$ is the predicted probability for $A$ from the first stage. Then the second stage model can be written as follows

$$
\operatorname{Pr}(Y=1)=\beta_{0}+\beta_{1 \text { Stage-2SRI }} A+\beta_{2} r
$$

and then $\hat{\beta}_{1 \text { Stage-2SRI }}$ is the estimated RD.

\section{Both-stages adjusted 2SRI}

The both-stages adjusted 2SRI is the same as naive 2SRI except it adjusts for $L$ in both stages of the model [22]. The same as naive and first-stage adjusted 2SRI, we used a logistic regression in the first stage of the model

$$
\operatorname{logit}(\operatorname{Pr}(A=1))=\gamma_{0}+\gamma_{1} Z+\gamma_{2} L_{1}+\gamma_{3} L_{2} .
$$

Then the second stage model can be written as follows

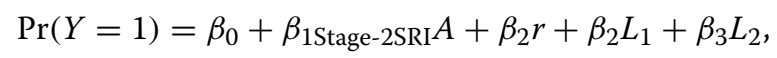

where $r=A-\widehat{A}$ is the residuals from the first stage with $\hat{A}$ is the predicted probability for $A$ from the first stage. Then $\hat{\beta}_{1 \text { Stage-2SRI }}$ is the estimated RD.

\section{$N P C B$}

This nonparametric method estimates the bounds for the effect of interest rather than a point estimate and is restricted to a binary outcome $(Y)$ with a binary or trinary instrument $[18,19]$. This method uses a constrained probability statement to provide bounds on the estimated treatment effect. But these bounds are the range of the true causal effect of interest, not the confidence interval $[18,19]$. The bounds for the RD can be written as

$$
\begin{gathered}
\left\{\begin{array}{c}
p_{00.0}+p_{11.1}-1 \\
p_{00.1}+p_{11.1}-1 \\
p_{11.0}+p_{00.1}-1 \\
p_{00.0}+p_{11.0}-1 \\
\max \\
2 p_{00.0}+p_{11.0}+p_{11.0}+p_{11.1}-2 \\
p_{00.0}+2_{p 11.0}+p_{00.1}+p_{01.1}-2 \\
p_{10.0}+p_{11.0}+2_{p 00.1}+p_{11.1}-2 \\
p_{00.0}+p_{01.0}+p_{00.1}+2 p_{11.1}-2
\end{array}\right\} \leq \mathrm{RD} \\
1-p_{10.0}-p_{01.1} \\
1-p_{01.0}-p_{10.1} \\
1-p_{01.0}-p_{10.0} \\
1-p_{01.1}-p_{10.1} \\
\leq \min \left\{\begin{array}{c} 
\\
2-2 p_{01.1}-p_{10.0}-p_{10.1}-p_{11.1} \\
2-p_{01.0}-2_{p 10.0}-p_{00.1}-p_{01.1} \\
2-p_{10.0}-p_{11.0}-2 p_{01.1}-p_{10.1} \\
2-p_{00.0}-p_{01.0}-p_{01.1}-2_{p 10.1}
\end{array}\right\},
\end{gathered}
$$

where $p_{y a . z}=\operatorname{Pr}(Y=y, A=a \mid Z=z)$ with $0 \leq p_{y a . z} \leq 1$ and $\sum_{y, a} p_{y a . z}=1[19]$.

\section{Assumptions of the estimation methods}

To estimate a causal effect, all models described in the Methods section assume consistency (i.e., the potential outcome under the observed treatment is the observed outcome), no interference (i.e., potential outcome for a subject does not depend on the treatment status of another subject), exchangeability (i.e., no unmeasured confounding), positivity (i.e., probability of receiving either treatment is greater than zero), and well-defined interventions (e.g., taking $5 \mathrm{mg}$ aspirin) [74, 75]. All parametric methods (all methods except the NPCB) further assume correct model specification [76]. The naive methods assume a random pattern of nonadherence [4]. The IV-based methods assume the IV is associated with the treatment (relevance assumption), there are no defiers (monotonicity assumption), and the IV affects the outcome only through its effect on treatment [77]. The third assumption is also known as the exclusion restriction. Additionally, the 2SRI assumes the linearity of residuals, i.e., residuals are linearly associated with the outcome of interest [10, 77]. Furthermore, all methods assume missing at random and no measurement error [78].

\section{Appendix B}

This section is for the results of sensitivity analyses. The DAGs are shown in Appendix C Fig. 25. Compared to the main text DAG, there is no measured confounder for these appendix DAGs. We consider these simplified scenarios to replicate the scenario for our case study. 


\section{Sensitivity analysis: exclusion restriction satisfied, unmeasured confounding present Setup}

This sensitivity analysis was done for a simplified version of DAG 2 in the main text, where the exclusion restriction is satisfied but unmeasured confounding is present. The DAG is shown in Appendix C Fig. 25(A). For this scenario, we simulated data from the following algorithms, where $A$ is the treatment, $Y$ is the outcome of interest, $Z$ is the randomization, and $U$ is unmeasured confounding.

$$
\begin{gathered}
Z \sim \operatorname{Bernoulli}(0.5) \\
U \sim \operatorname{Bernoulli}(0.5) \\
A \sim \operatorname{Bernoulli}\left(p_{A}\right) \text { with } p_{A}=\alpha_{0}+\alpha_{1} Z+\alpha_{4} U \\
Y \sim \operatorname{Bernoulli}\left(p_{Y}\right) \text { with } p_{Y}=\theta_{0}+\theta_{1} A+\theta_{4} U+\theta_{5} Z .
\end{gathered}
$$

In Eqn. (2), $\alpha_{0}$ is associated with the nonadherence rate, $\alpha_{1}=0.1$ is the coefficient associated with $Z, \alpha_{4}=0.1$ is the coefficient associated with $U ; \theta_{0}$ is associated with the event rate, $\theta_{1}$ is the treatment effect of interest, and $\theta_{4}$ determines the strength of confounding, and $\theta_{5}$ determines the strength of the direct effect of IV assumption of exclusion restriction violation. Under different choices of $\alpha_{0}$, we considered six levels of nonadherence: $10,20,40$, 60,80 , and $90 \%$.

We set $\theta_{0}=0.2$, and $\theta_{5}=0$ (i.e., no violation of exclusion restriction assumption). For each of the six nonadherence scenarios $\left(\alpha_{0}\right)$, we considered five scenarios of the treatment effect of interest $\left(\theta_{1}\right)$ and two versions of confounding $\left(\theta_{4}\right)$, making a total of 60 scenarios. We set the treatment effect of interest as $\theta_{1}=$ $\{-0.2,-0.05,0,0.05,0.2\}$ and confounding as $\theta_{4}=0.1$ and 0.5 respectively for weak and strong confounding.

\section{Results}

Since there was no measured confounder $(L)$ associated with our data generating mechanism here, there are no baseline adjusted methods as well as first-stage adjusted or both-stages adjusted 2SLS and 2SRI methods. Instead, we reported the results for the ITT, naive $\mathrm{PP}$, naive $\mathrm{AT}$, naive 2SLS, naive 2SRI, and NPCB methods.

Under the null treatment effect scenario with weak confounding, all methods produce small bias (Appendix $\mathrm{C}$ Fig. 26). In the presence of strong confounding, the naive PP and AT produce slightly large bias. The amount of bias remains approximately the same for the non-null treatment effect. The ITT method produces a very small bias for the null effect, while it performs the worst when the treatment effect is non-null. In contrast, the 2SLS and 2SRI produce unbiased estimates in all scenarios.

Under the null treatment effect scenario, all methods produce small MSE in the presence of weak confounding, while naive PP shows higher MSE beyond 60\% of nonadherence (Appendix C Fig. 27). Under the non-null effect, we observed a similar pattern of MSE except for the ITT. The ITT method produces very high MSE regardless of weak or strong confounding.

Appendix C Fig. 28 shows the 95\% coverage probability for different nonadherence rates using Appendix DAG 1. As expected, the ITT method has the highest coverage for the null treatment effect scenario, while it has minimal coverage for the non-null effect. The naive PP and AT methods also produce noticeable small coverage when there is weak confounding, but these methods produce very small coverage under the strong confounding scenarios. On the other hand, naive 2SLS and 2SRI consistently have very high coverage under both null or nonnull effects and weak or strong unmeasured confounding scenarios.

The NPCB method produces a wider bound for both the null and the non-null effect, regardless of the weak or strong confounding (Appendix C Fig. 29). However, the width of bounds is only smaller for $10 \%$ and $90 \%$ nonadherence.

\section{Sensitivity analysis: violation of the exclusion restriction Setup}

In this example, the exclusion restriction is violated $(Z$ directly affects $Y$ ) and there exists an unmeasured confounder $U$. The DAG is shown in Appendix C Fig. 25(B). We used Eqn. (2) to generate the data for Appendix DAG 2. We set $\theta_{0}=0.2, \theta_{1}=0.05$, two versions of $\theta_{4}$, and two versions of $\theta_{5}$ with $10,20,40,60,80$, and $90 \%$ nonadherence, making a total of 24 scenarios. We set $\theta_{5}$ $=0.2$ meaning that the exclusion restriction of the IV assumption violation is larger and $\theta_{5}=0.05$ indicating the violation is smaller. The same as Appendix DAG 1, we set $\theta_{4}=0.1$ and 0.5 for weak and strong confounding, respectively.

\section{Results}

Since there was no measured confounder $(L)$ associated with our data generating mechanism here, there are no baseline adjusted methods as well as first-stage adjusted or both-stages adjusted 2SLS and 2SRI methods. Instead, we reported the results for the ITT, naive PP, naive AT, naive 2SLS, naive 2SRI, and NPCB methods.

Appendix C Fig. 30 shows the bias versus nonadherence rate using Appendix DAG 2. Appendix C Fig. 30(A) and (B) show the results when the violation of the exclusion restriction assumption is minor, and Appendix $C$ Fig. $30(C)$ and (D) show the results when violation is severe. We observed that the 2SLS and 2SRI methods produce a greater bias when the exclusion restriction assumption is violated. The bias is more pronounced when the violation of exclusion restriction assumption is severe. 


\section{Appendix C}

This section contains all the supplementary figures considered in this study.

\section{Supplementary figures for simulation setting 1}
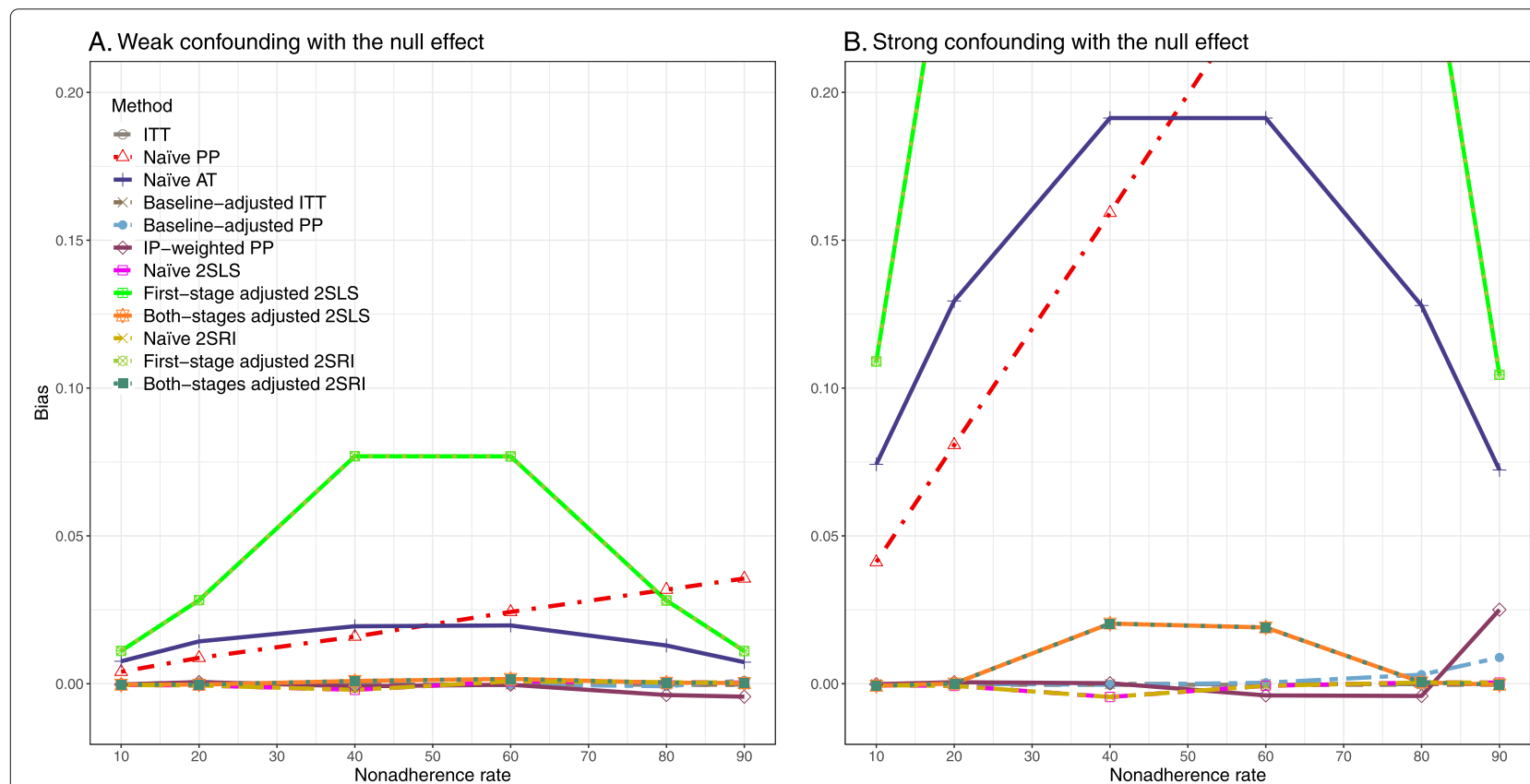

Fig. 6 Bias versus the nonadherence rate using DAG 1. The naive 2SLS and 2SRI share the same line, the first stage adjusted 2SLS and 2SRI share the same line, and both-stages adjusted 2SLS and 2SRI share the same line as they produce the same amount of bias. The first stage adjusted 2SLS and 2SRI, and naive PP produce larger than 0.20 bias for strong unmeasured confounders so that the bias is out of the bound [0, 0.20]. Abbreviations: ITT: intention-to-treat, PP: per-protocol, AT: as-treated, IP-weighted PP: inverse probability-weighted per-protocol, 2SLS: two-stage least square, 2SRI: two-stage residual inclusion 

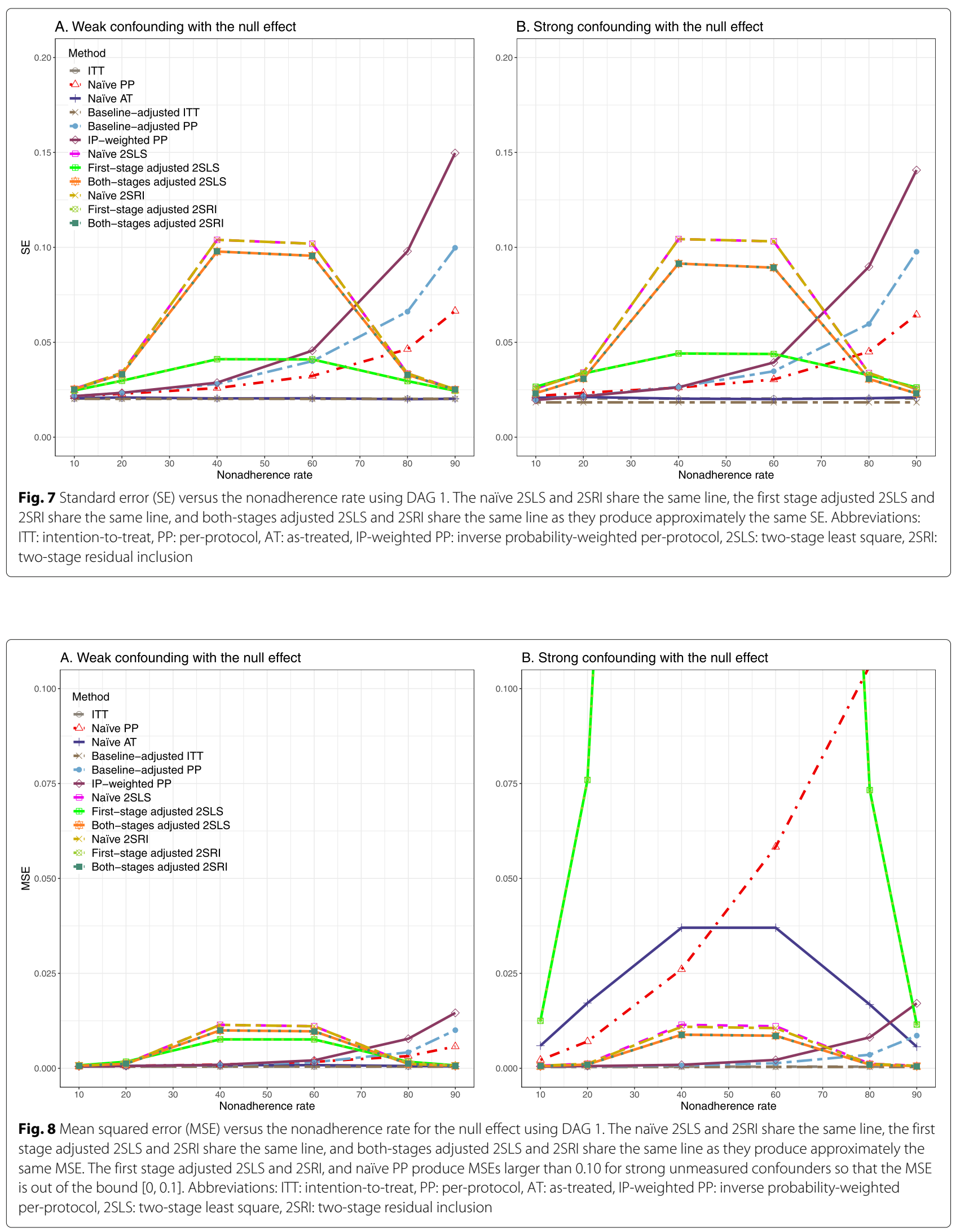

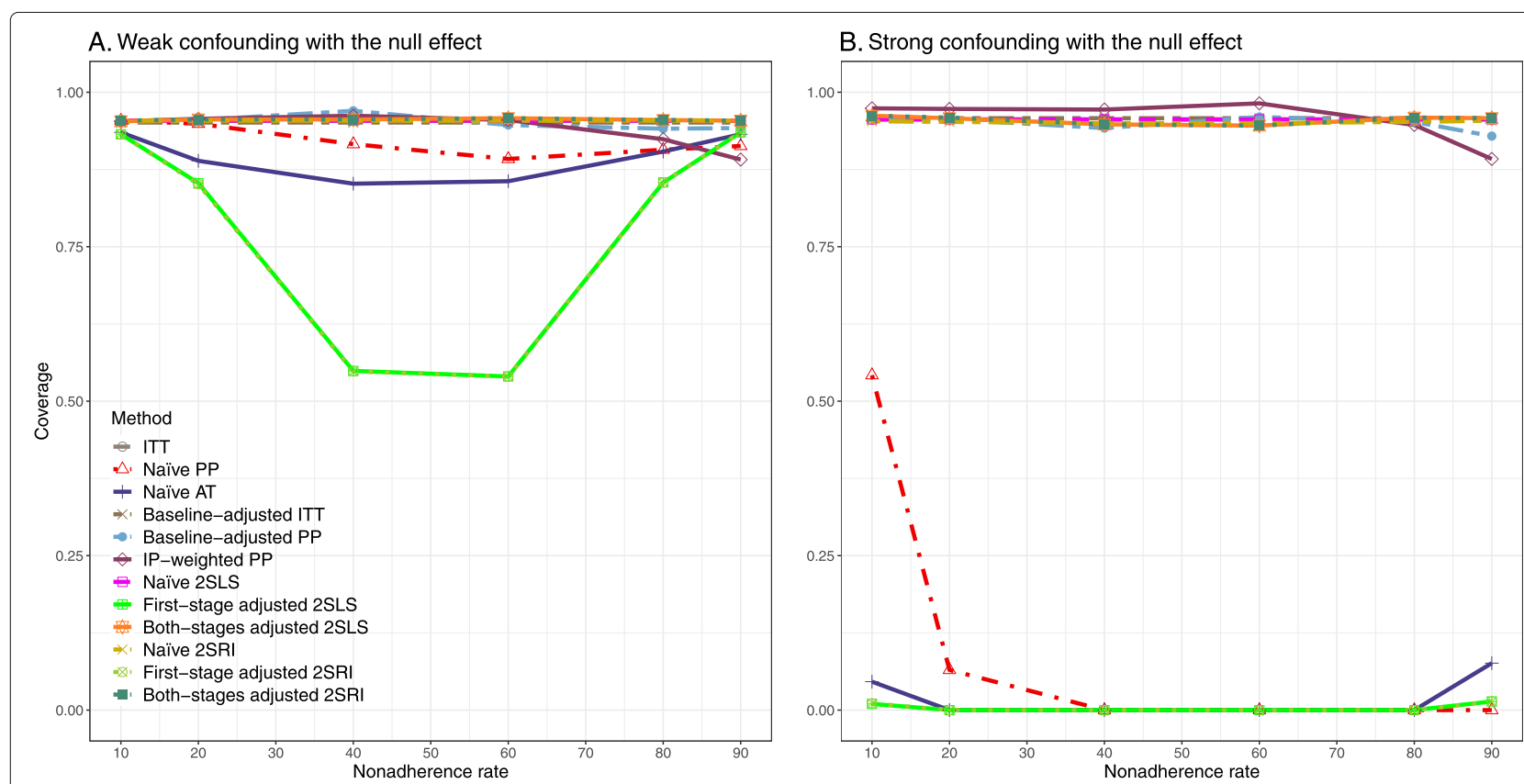

Fig. 9 95\% coverage probability versus the nonadherence rate using DAG 1. The naïve 2SLS and 2SRI share the same line, the first-stage adjusted 2SLS and 2SRI share the same line, and both-stages adjusted 2SLS and 2SRI share the same line as they produce approximately the same coverage probability. Abbreviations: ITT: intention-to-treat, PP: per-protocol, AT: as-treated, IP-weighted PP: inverse probability-weighted per-protocol, 2SLS: two-stage least square, 2SRI: two-stage residual inclusion
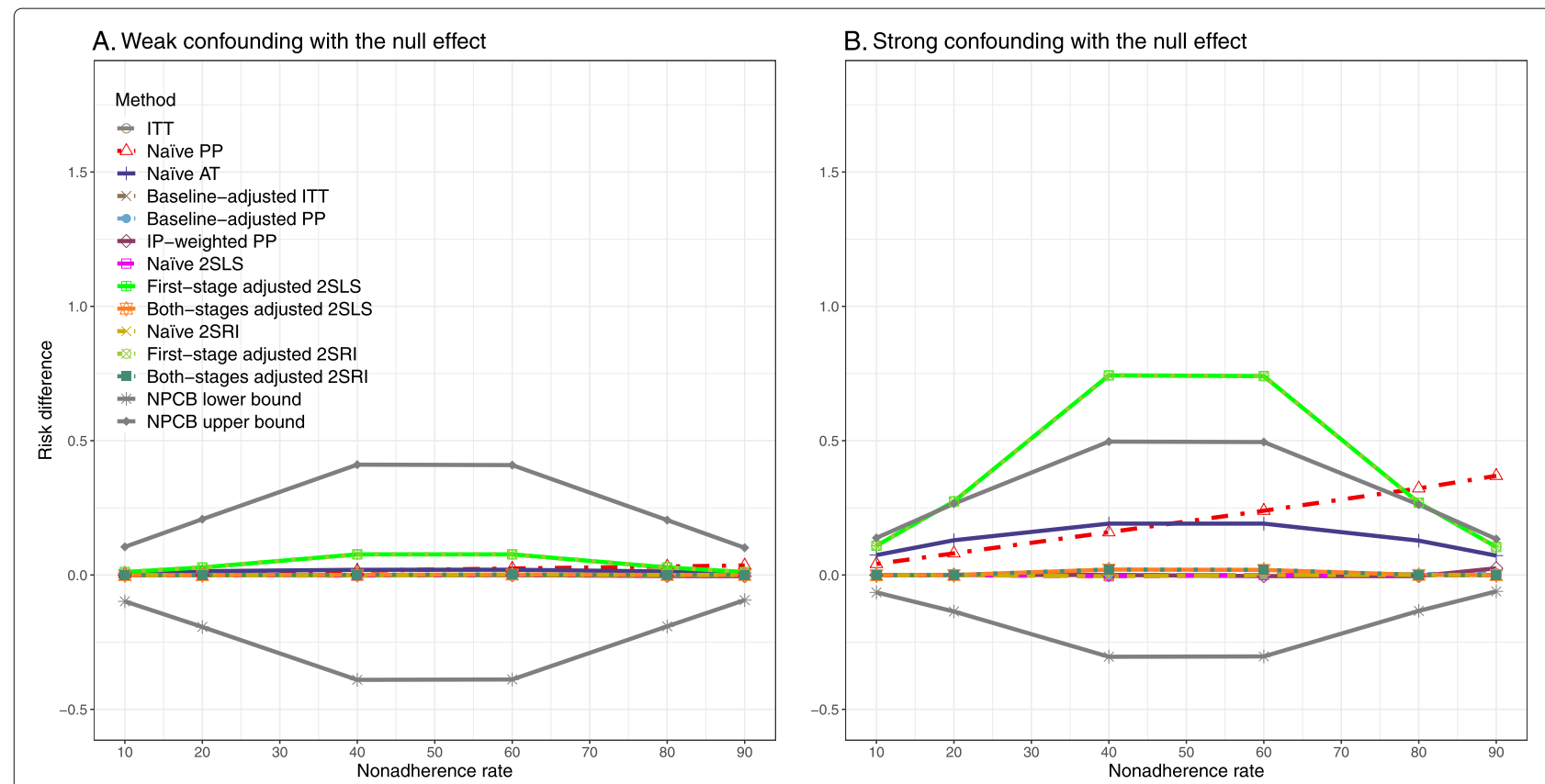

Fig. 10 Mean risk difference (RD) versus the nonadherence rate for the null effect using DAG 1. The grey lines are the lower and upper bound of the NPCB method. The naïve 2SLS and 2SRI share the same line, the first stage adjusted 2SLS and 2SRI share the same line, and both-stages adjusted 2SLS and 2SRI share the same line as they produce the same RD estimate. Abbreviations: ITT: intention-to-treat, PP: per-protocol, AT: as-treated, IP-weighted PP: inverse probability-weighted per-protocol, 2SLS: two-stage least square, 2SRI: two-stage residual inclusion, NPCB: nonparametric causal bound 


\section{Supplementary figures for simulation setting 2}
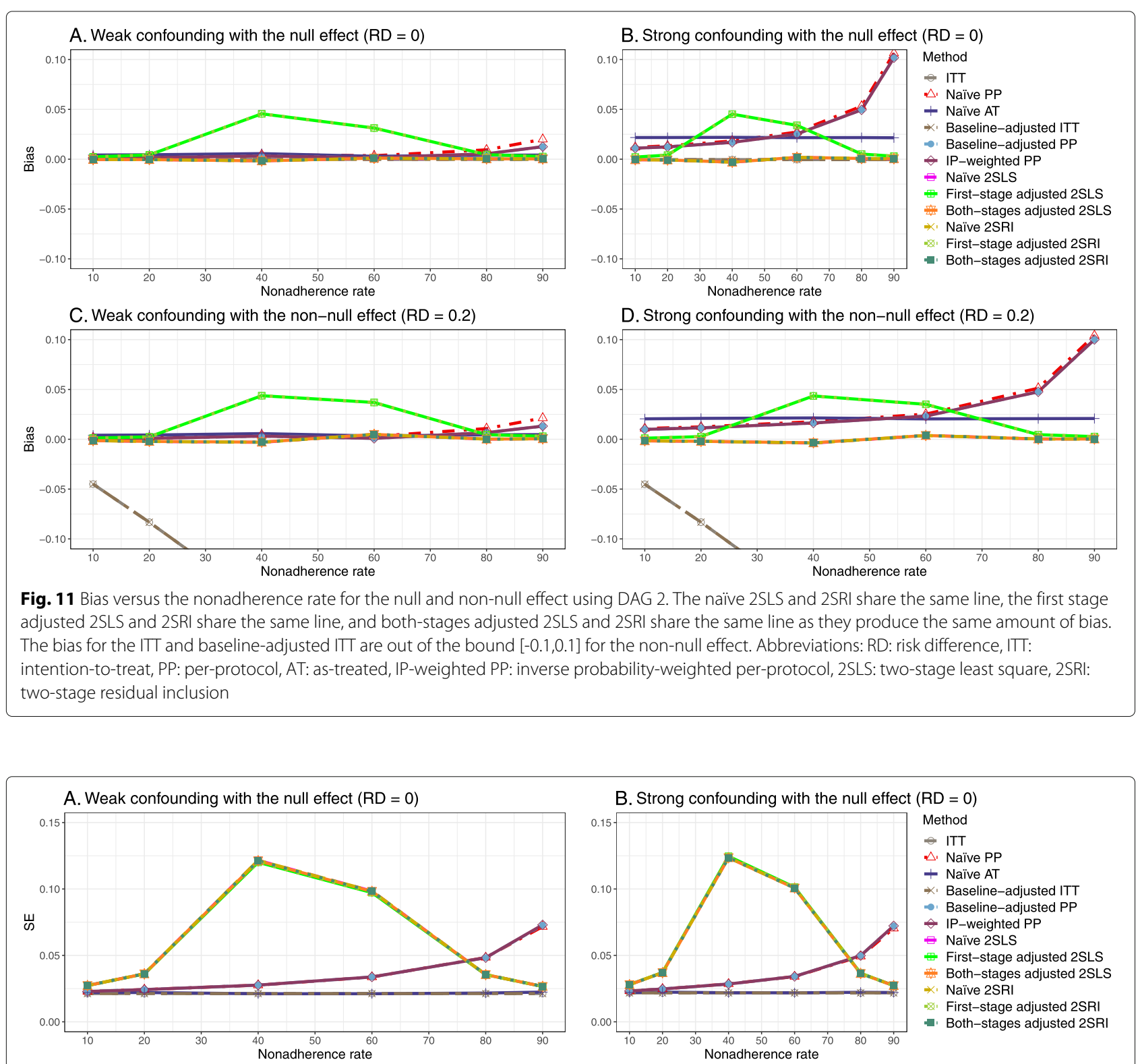

C. Weak confounding with the non-null effect $(R D=0.2)$

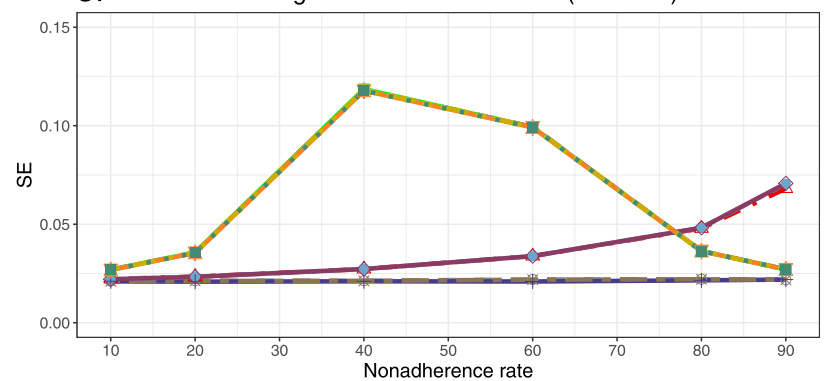

D. Strong confounding with the non-null effect $(\mathrm{RD}=0.2)$

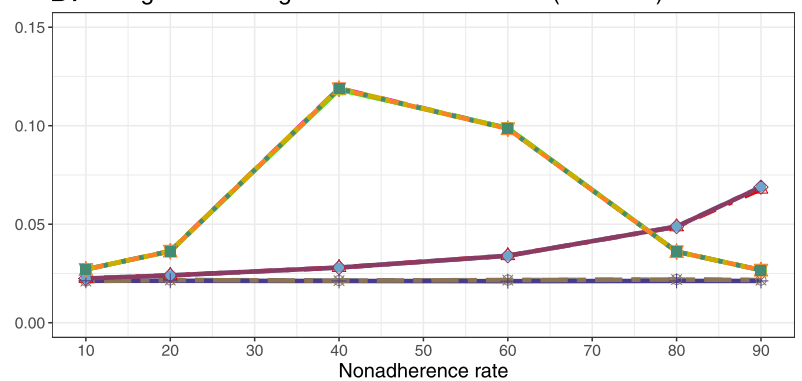

Fig. 12 Standard error (SE) versus the nonadherence rate for null and non-null effect using DAG 2. All 2SLS and 2SRI methods share the same line as they produce approximately the same SE. The PP methods also share approximately the same line. Abbreviations: RD: risk difference, ITT: intention-to-treat, PP: per-protocol, AT: as-treated, IP-weighted PP: inverse probability-weighted per-protocol, 2SLS: two-stage least square, 2SRI: two-stage residual inclusion 

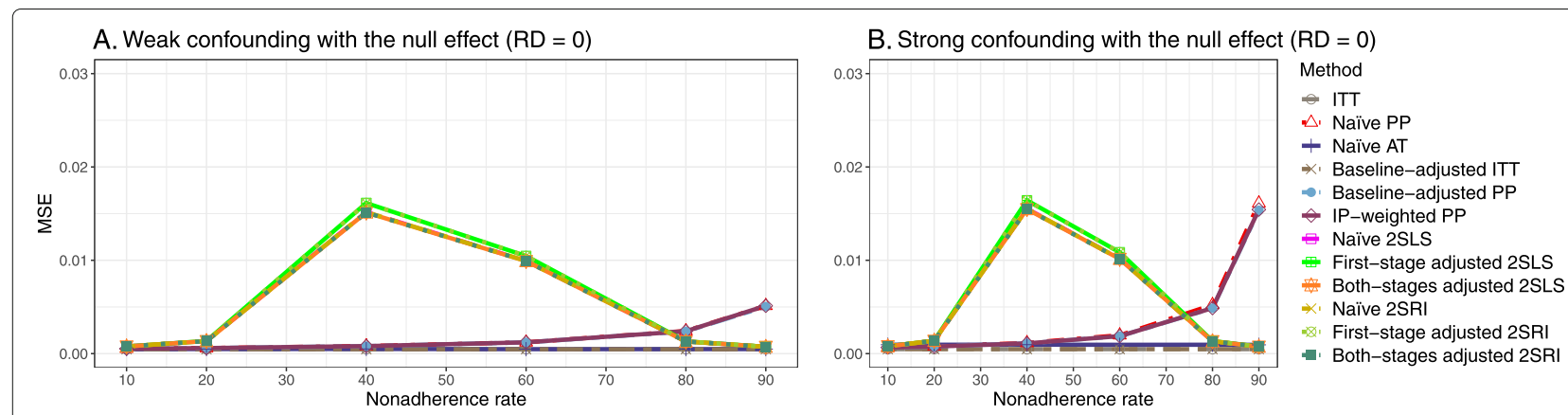

C. Weak confounding with the non-null effect $(R D=0.2)$

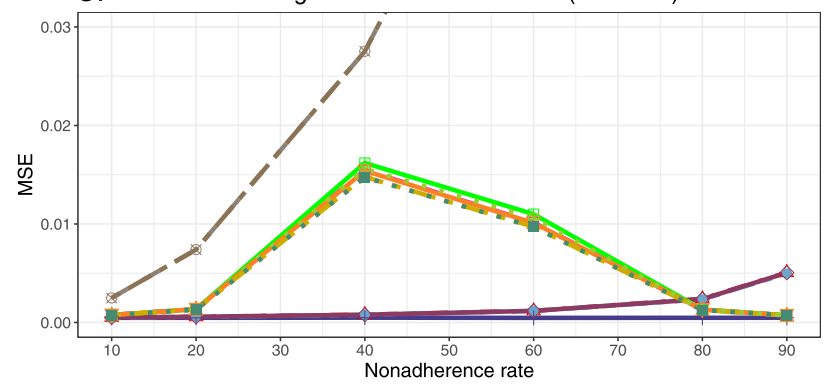

D. Strong confounding with the non-null effect $(\mathrm{RD}=0.2)$

Fig. 13 Mean squared error (MSE) versus the nonadherence rate for null and non-null effect using DAG 2. The 2SLS and 2SRI share the same line as they produce approximately the same amount of MSE. The ITT and baseline-adjusted ITT produce MSEs larger than 0.03 for the non-null effect scenario so that the MSE is out of the bound [0, 0.03]. Abbreviations: RD: risk difference, ITT: intention-to-treat, PP: per-protocol, AT: as-treated, IP-weighted PP: inverse probability-weighted per-protocol, 2SLS: two-stage least square, 2SRI: two-stage residual inclusion

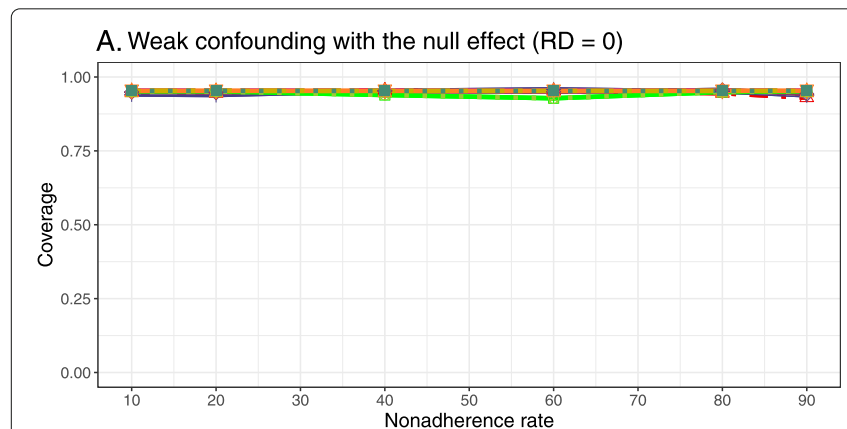

C. Weak confounding with the non-null effect $(\mathrm{RD}=0.2)$

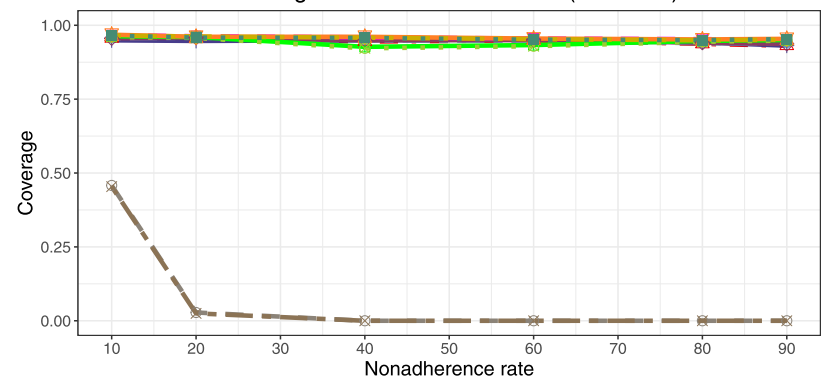

\section{B. Strong confounding with the null effect $(\mathrm{RD}=0)$}

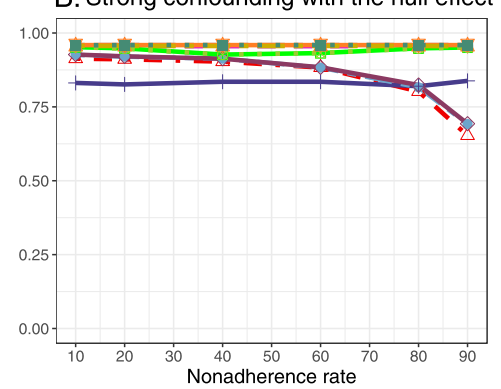

Method

$\Leftrightarrow$ ITT

$\triangle$ Naïve PP

+ Naïve AT

$* *$ Baseline-adjusted ITT

$\rightarrow$ Baseline-adjusted PP

$\Leftrightarrow \mathrm{IP}$-weighted PP

- Naïve 2SLS

- First-stage adjusted 2SLS

Both-stages adjusted 2SLS

$\Rightarrow$ ‘’ Naïve 2SRI

- First-stage adjusted 2SRI

- Both-stages adjusted 2SRI

D. Strong confounding with the non-null effect $(\mathrm{RD}=0.2)$

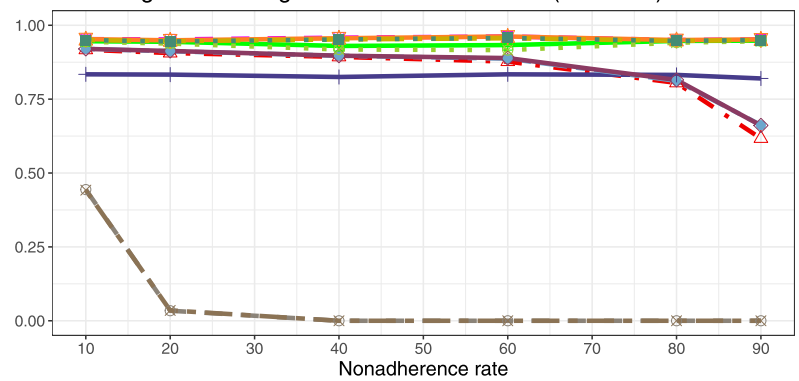

Fig. 1495 percentage coverage probability versus the nonadherence rate for the null and non-null effect using DAG 2. The naive and both-stages adjusted 2SLS and 2SRI share the same line as they produce approximately the same coverage probability. Abbreviations: RD: risk difference, ITT: intention-to-treat, PP: per-protocol, AT: as-treated, IP-weighted PP: inverse probability-weighted per-protocol, 2SLS: two-stage least square, 2SRI: two-stage residual inclusion 


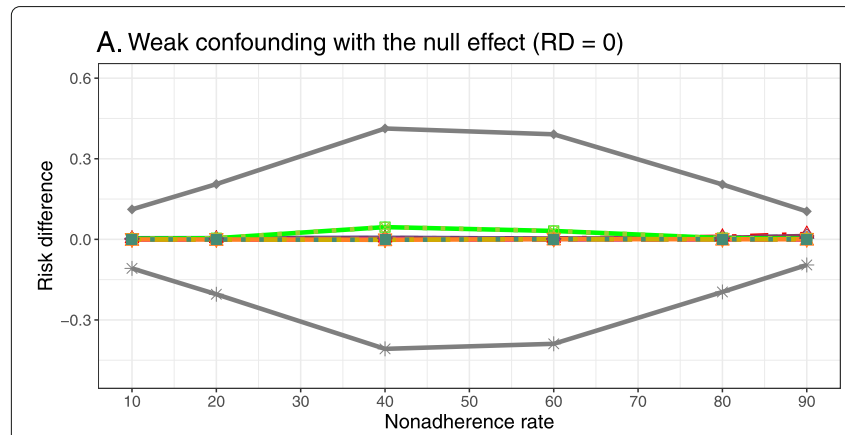

C. Weak confounding with the non-null effect $(R D=0.2)$

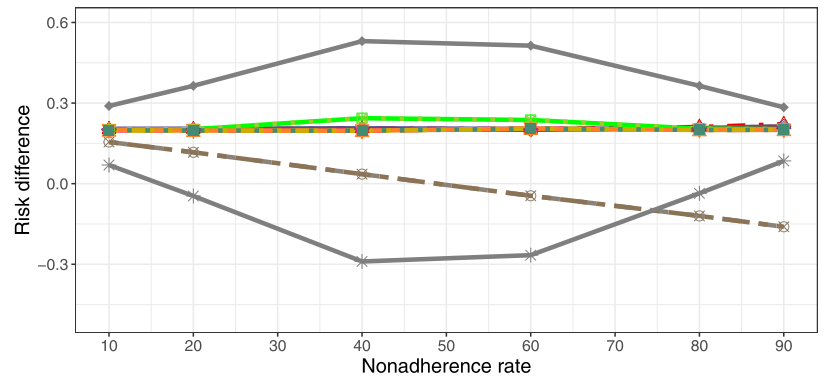

B. Strong confounding with the null effect $(\mathrm{RD}=$ Ohethod

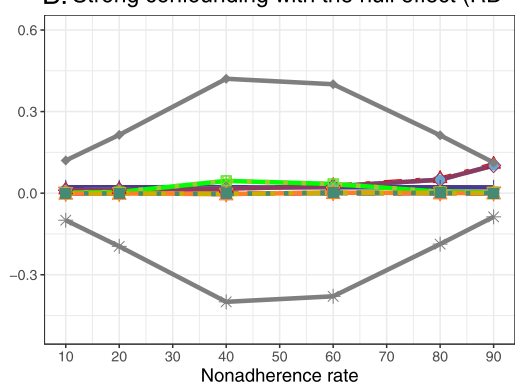

$\because \mathrm{ITT}$

$\triangle$ Naïve PP

+ Naïve AT

$*$ Baseline-adjusted ITT

$\rightarrow$ Baseline-adjusted PP

$\Leftrightarrow \mathrm{IP}$-weighted PP

- Naïve 2SLS

First-stage adjusted 2SLS

Both-stages adjusted 2SLS

* Naïve 2SRI

$*$ Nairve 2SRI
First-stage adjusted 2SRI

Both-stages adjusted 2SRI

* NPCB lower bound

$\div$ NPCB upper bound

D. Strong confounding with the non-null effect $(R D=0.2)$

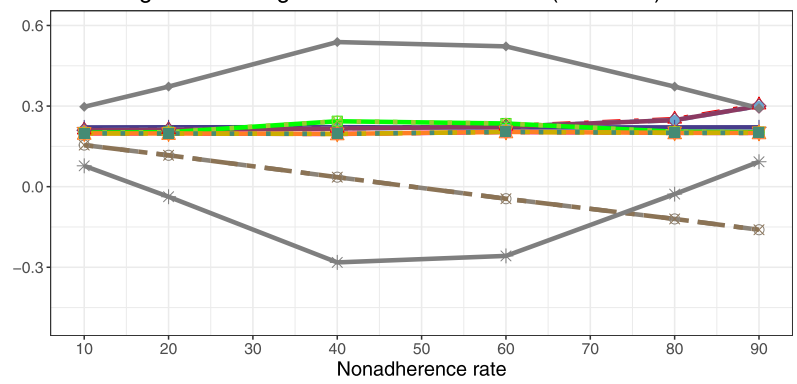

Fig. 15 Mean risk difference (RD) versus the nonadherence rate for DAG 2. The grey lines are the lower and upper bound of the NPCB method. The ITT and baseline-adjusted ITT superimposed on each other. Abbreviations: RD: risk difference, ITT: intention-to-treat, PP: per-protocol, AT: as-treated, IP-weighted PP: inverse probability-weighted per-protocol, 2SLS: two-stage least square, 2SRI: two-stage residual inclusion, NPCB: nonparametric causal bound

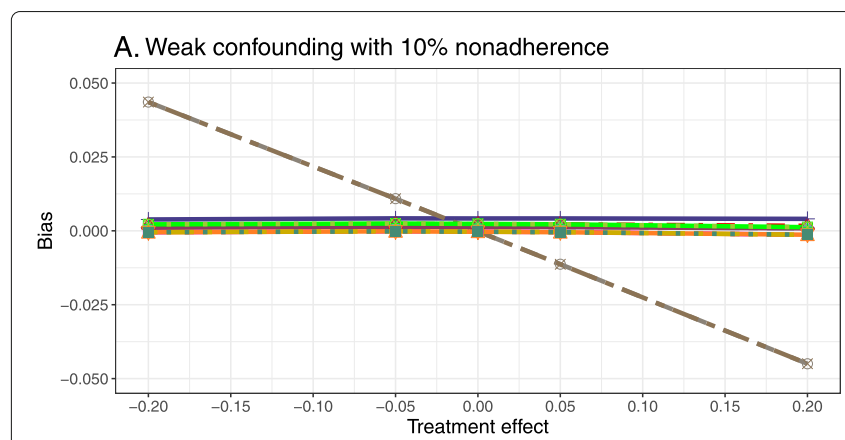

C. Weak confounding with $40 \%$ nonadherence

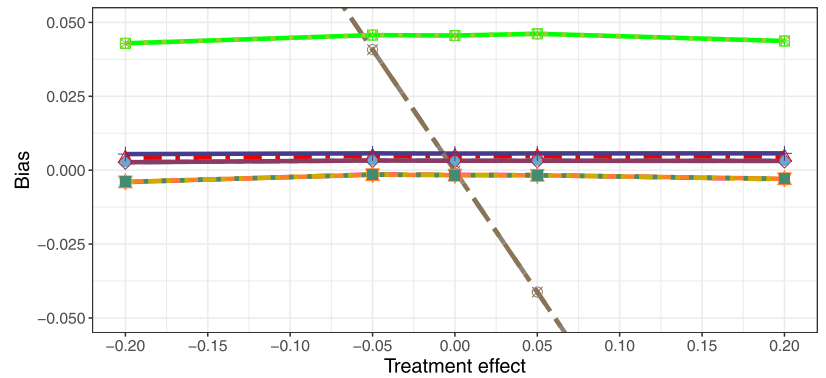

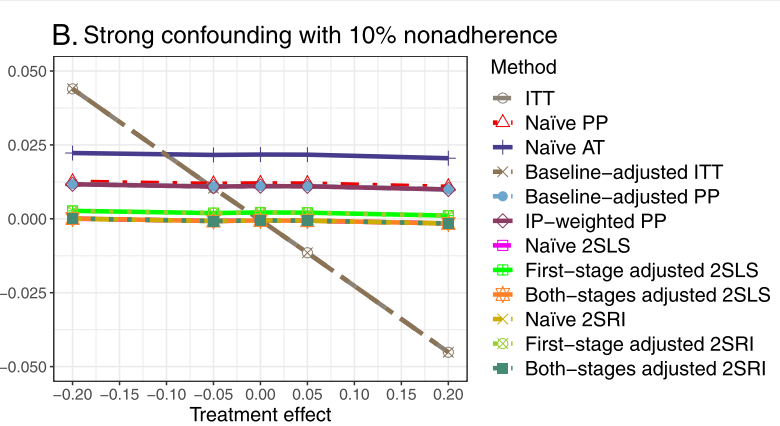

D. Strong confounding with $40 \%$ nonadherence

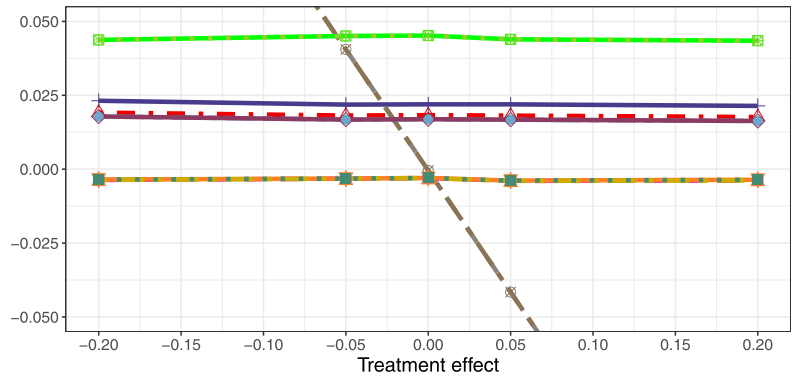

Fig. 16 Bias versus treatment effect in risk difference for 10 percent and 40 percent nonadherence using DAG 2. The naive and both-stages adjusted 2SLS and 2SRI share the same line as they produce the same amount of bias. The first-stage adjusted 2SLS and 2SRI methods also share the same line. The ITT and baseline-adjusted ITT produce high bias for 40 percent nonadherence so that the bias is out of the bound [-0.05, 0.05]. Abbreviations: ITT: intention-to-treat, PP: per-protocol, AT: as-treated, IP-weighted PP: inverse probability-weighted per-protocol, 2SLS: two-stage least square, 2SRI: two-stage residual inclusion 

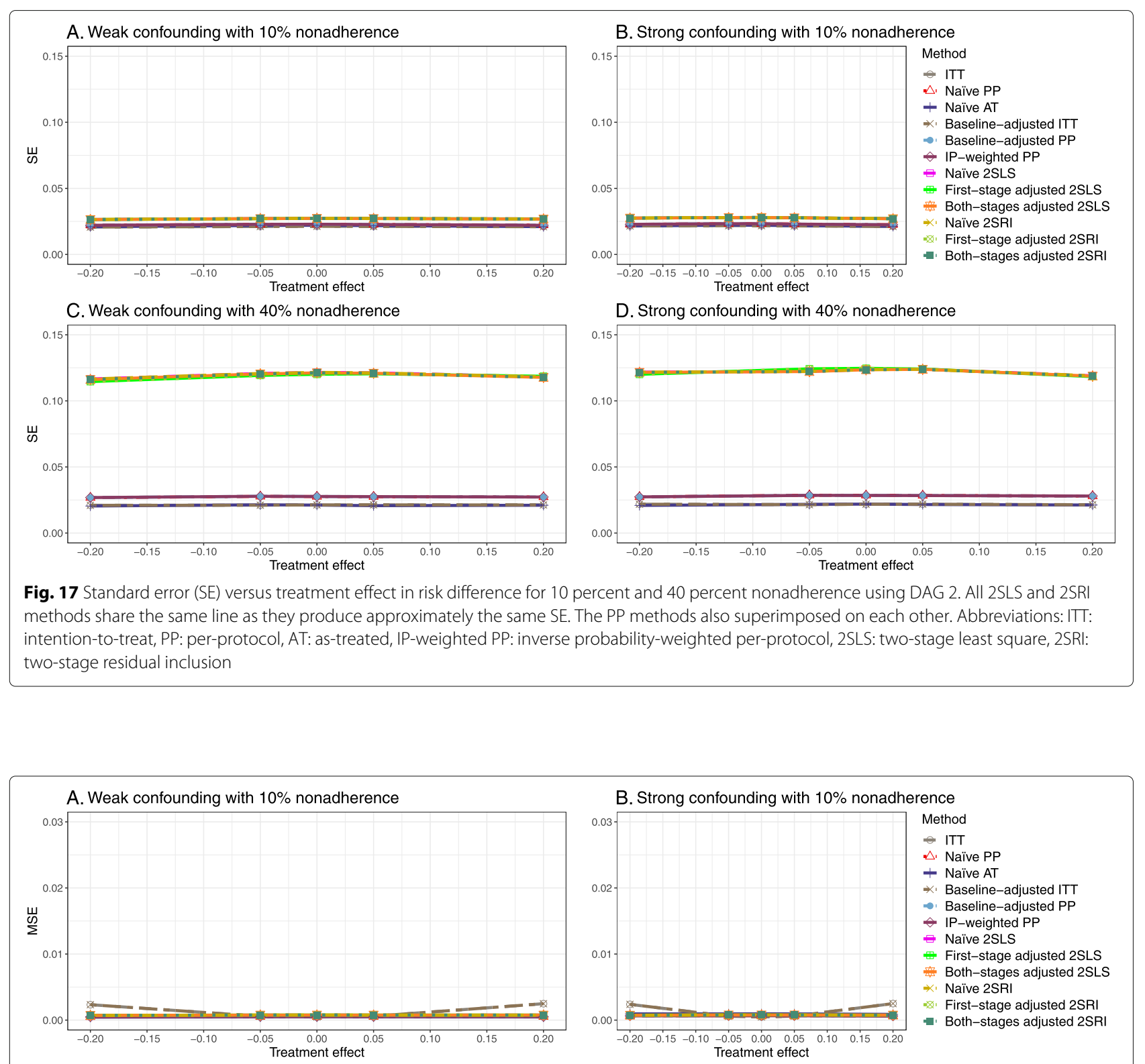

C. Weak confounding with $40 \%$ nonadherence

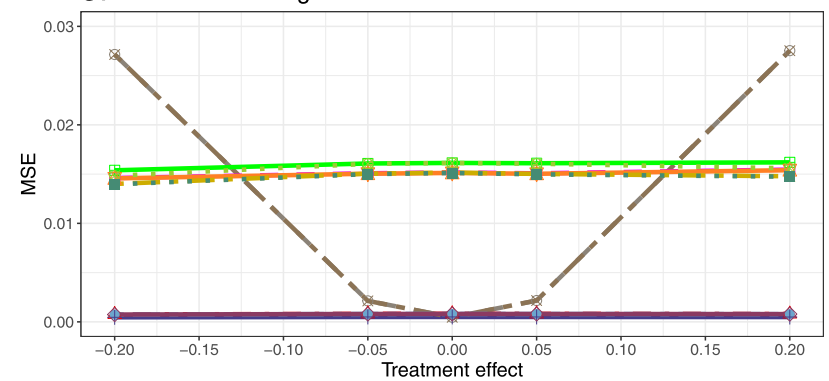

D. Strong confounding with $40 \%$ nonadherence

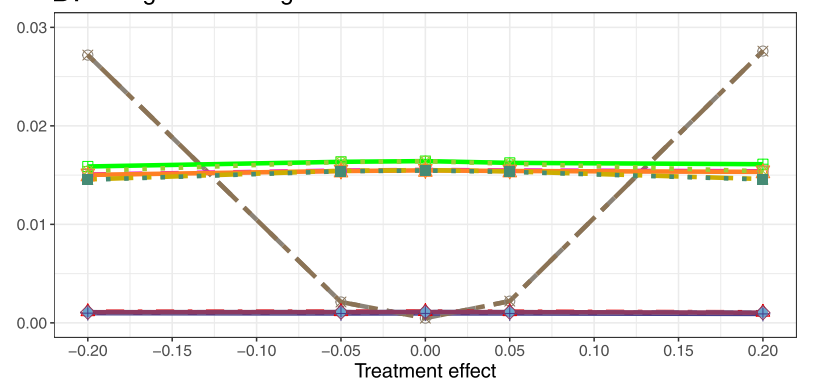

Fig. 18 Mean squared error (MSE) versus treatment effect in risk difference for 10 percent and 40 percent nonadherence using DAG 2. Abbreviations: ITT: intention-to-treat, PP: per-protocol, AT: as-treated, IP-weighted PP: inverse probability-weighted per-protocol, 2SLS: two-stage least square, 2SRI: two-stage residual inclusion 


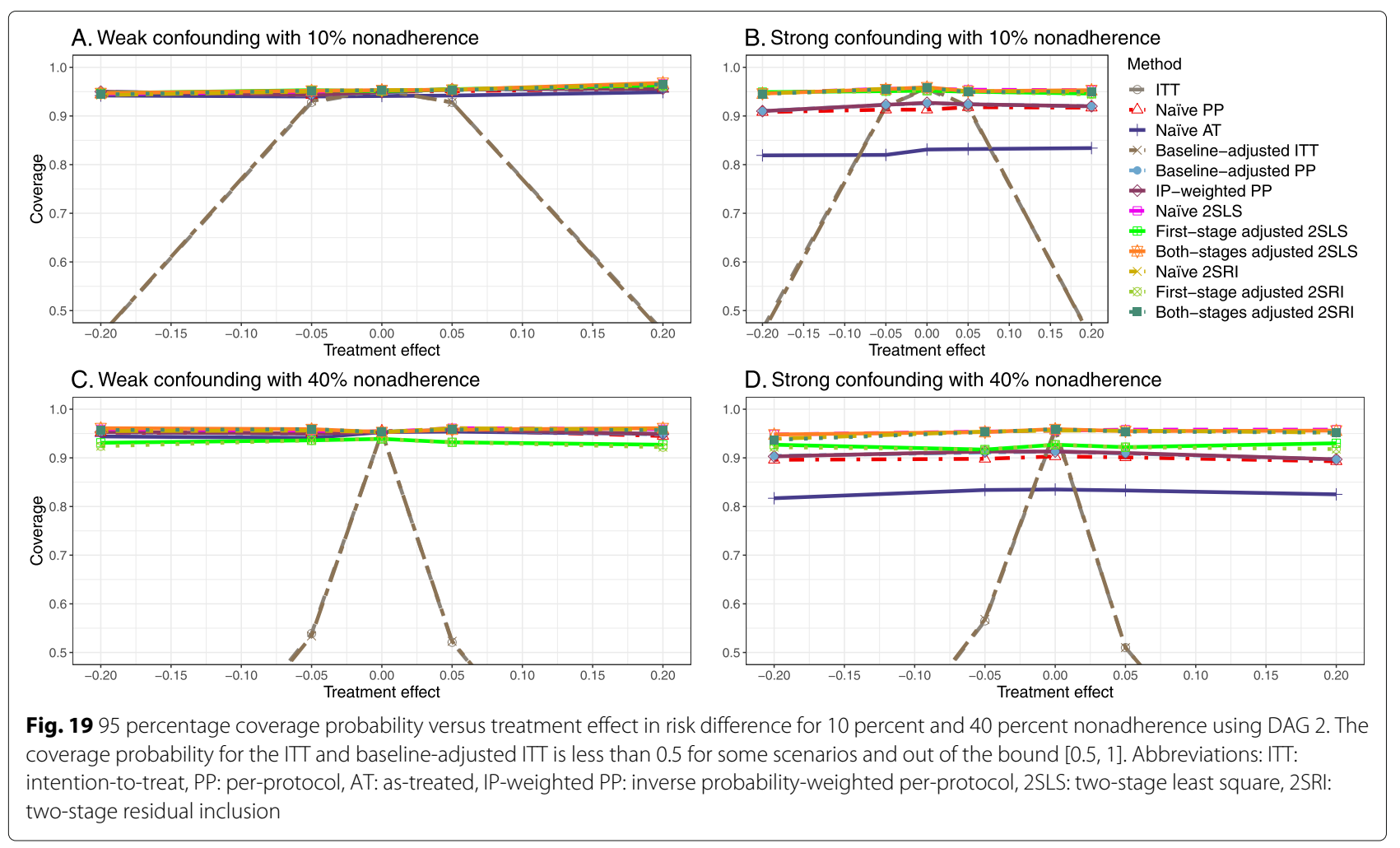

Supplementary figures for simulation setting 3

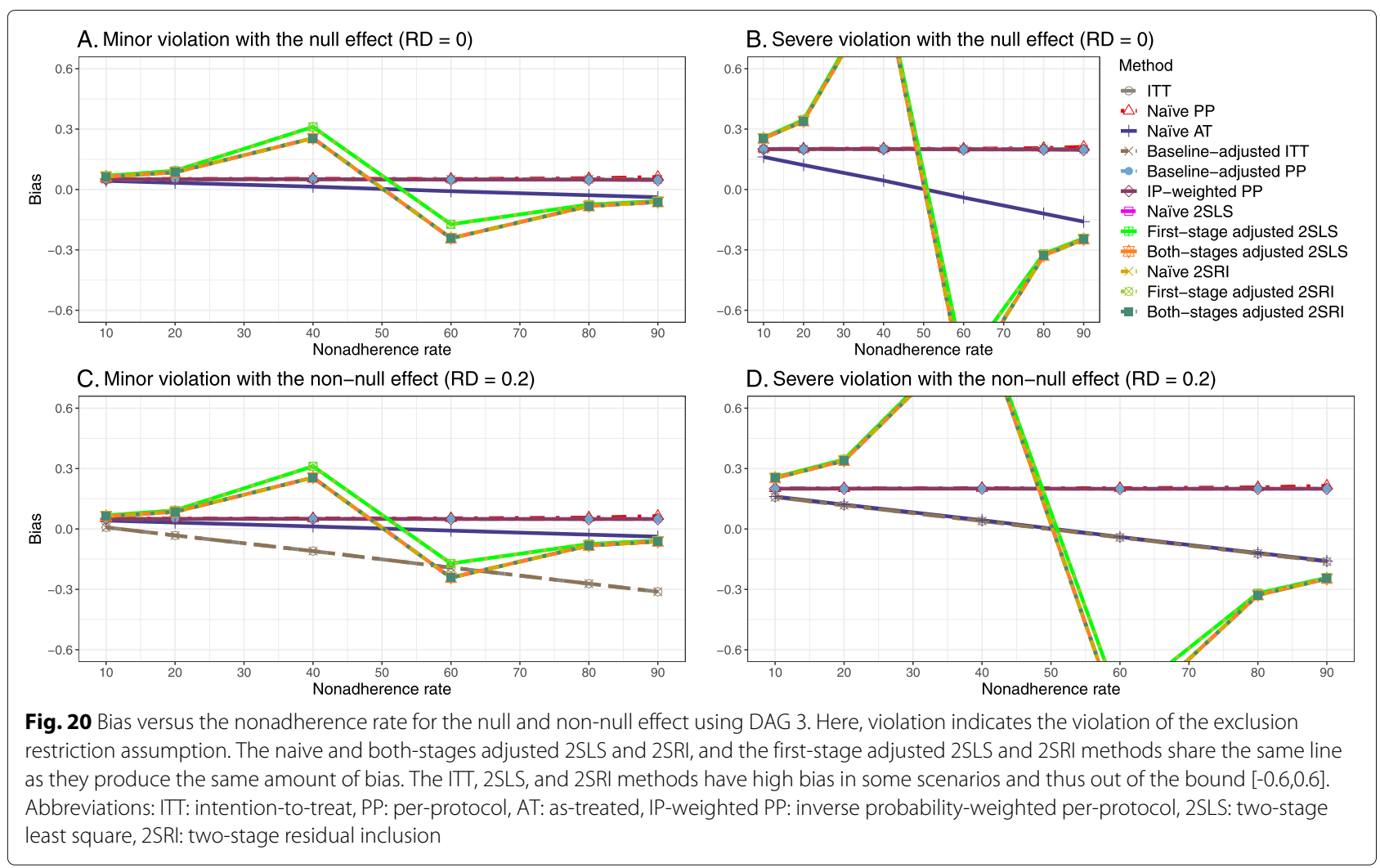



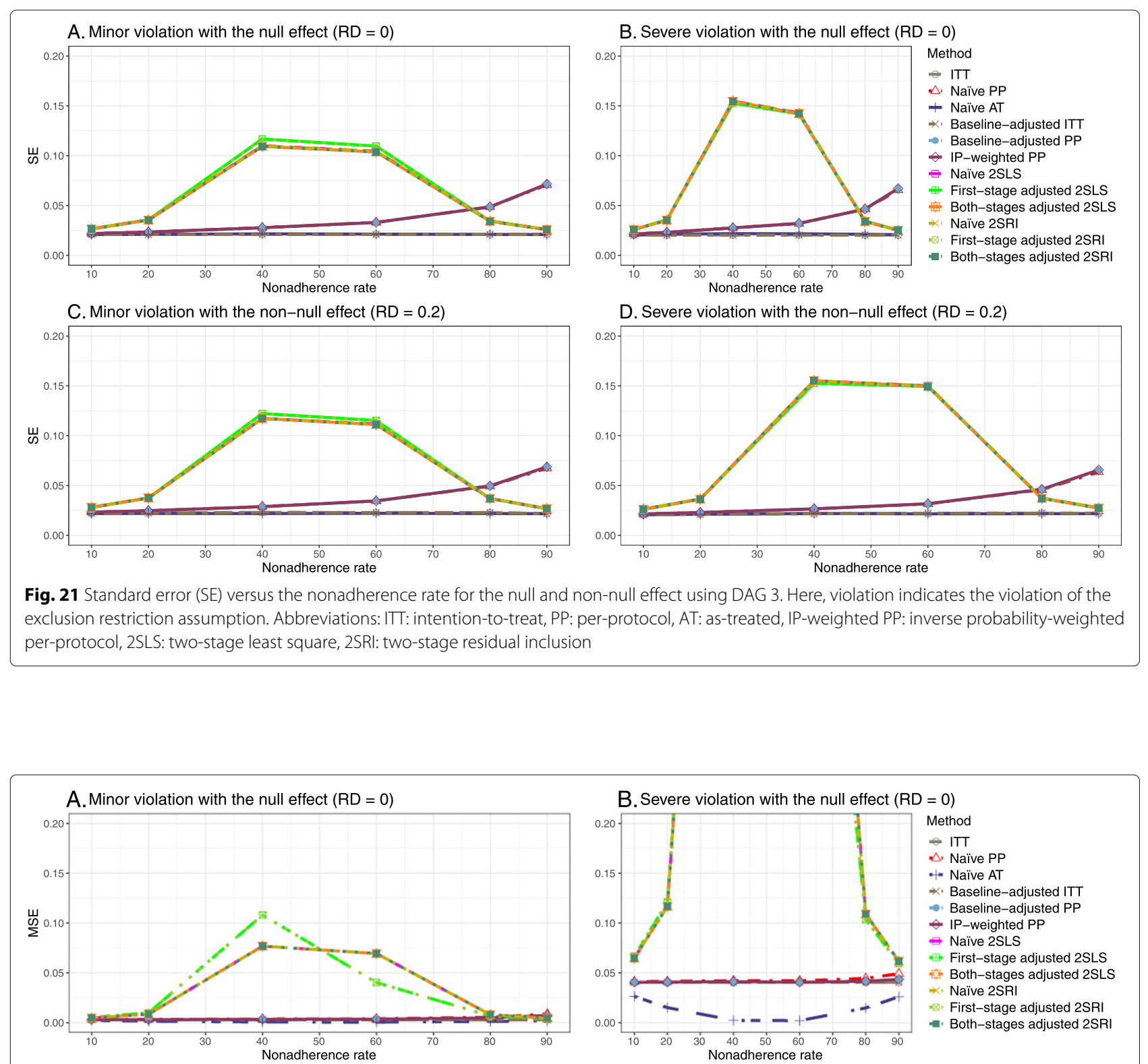

C. Minor violation with the non-null effect $(\mathrm{RD}=0.2)$

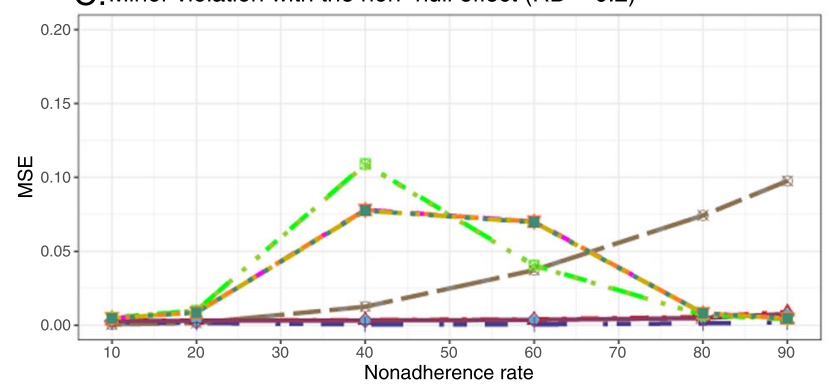

D. Severe violation with the non-null effect $(R D=0.2)$

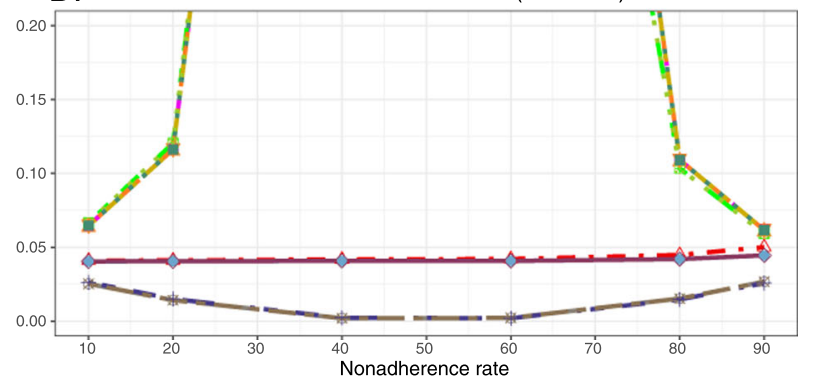

Fig. 22 Mean squared error (MSE) versus the nonadherence rate for the null and non-null effect using DAG 3. Here, violation indicates the violation of the exclusion restriction assumption. Abbreviations: ITT: intention-to-treat, PP: per-protocol, AT: as-treated, IP-weighted PP: inverse probability-weighted per-protocol, 2SLS: two-stage least square, 2SRI: two-stage residual inclusion 

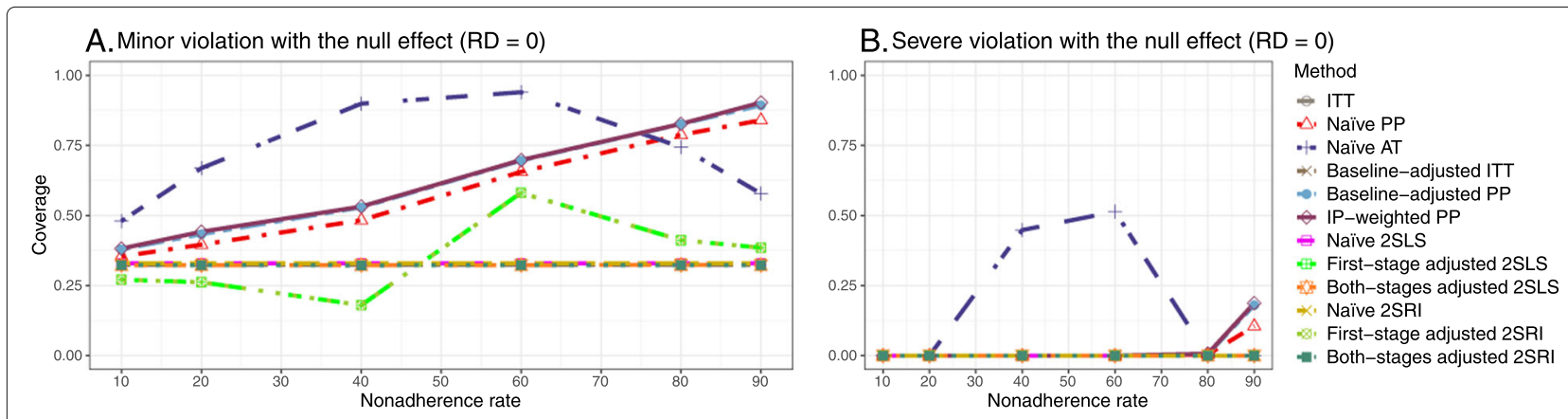

C. Minor violation with the non-null effect $(\mathrm{RD}=0.2)$

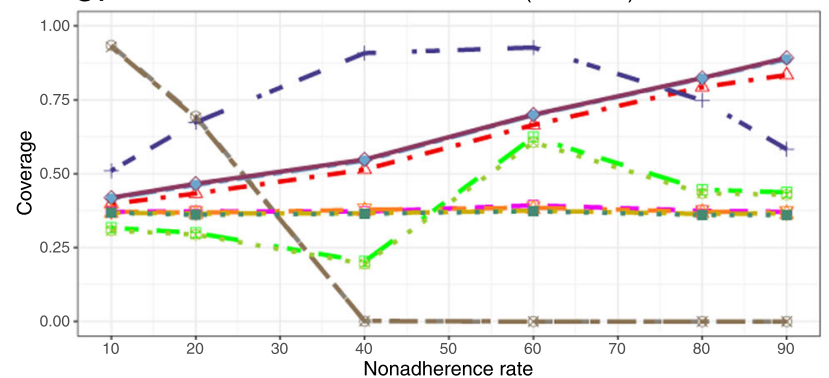

D. Severe violation with the non-null effect $(\mathrm{RD}=0.2)$

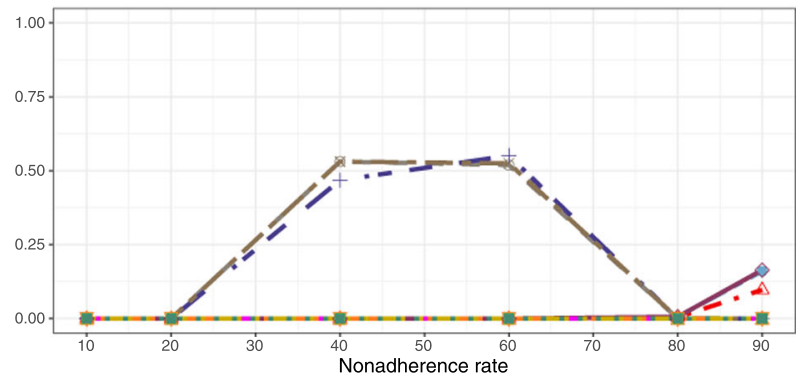

Fig. 2395 percentage coverage probability versus the nonadherence rate for the null and non-null effect using DAG 3. Here, violation indicates the violation of the exclusion restriction assumption. Abbreviations: ITT: intention-to-treat, PP: per-protocol, AT: as-treated, IP-weighted PP: inverse probability-weighted per-protocol, 2SLS: two-stage least square, 2SRI: two-stage residual inclusion
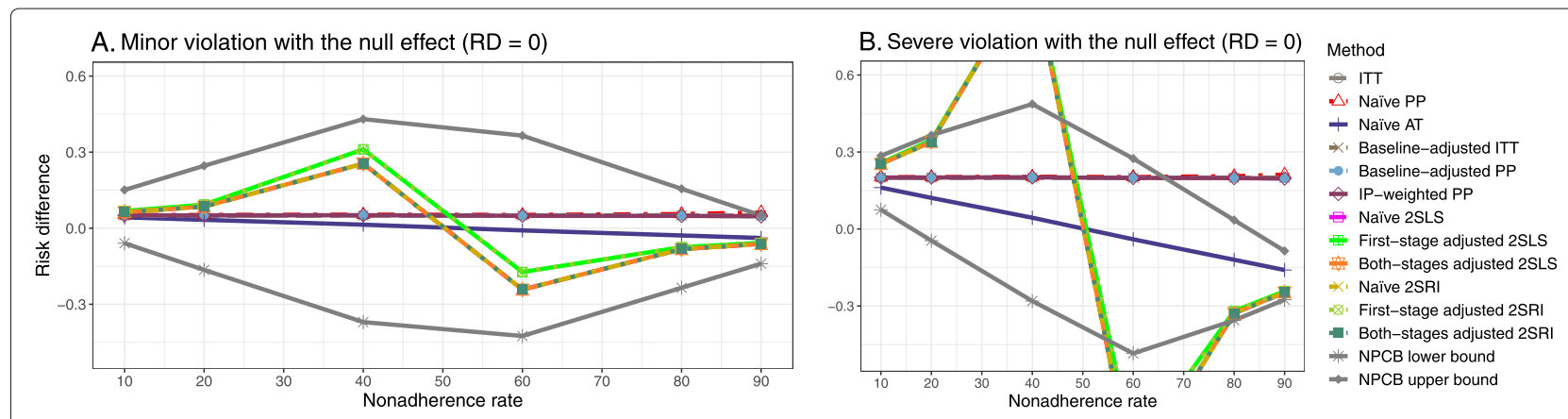

C. Minor violation with the non-null effect $(\mathrm{RD}=0.2)$

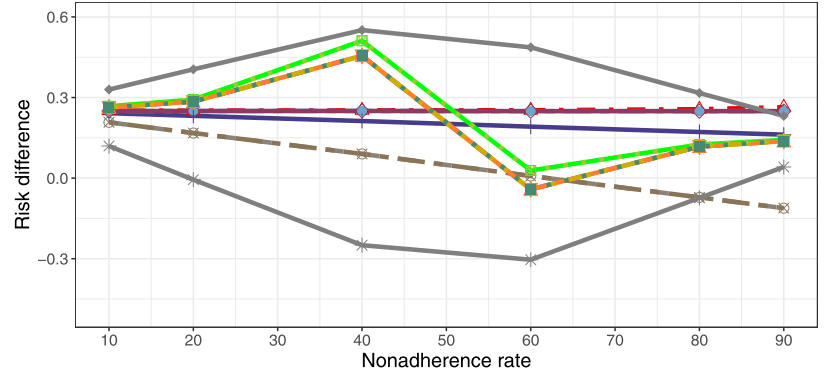

D. Severe violation with the non-null effect $(\mathrm{RD}=0.2)$

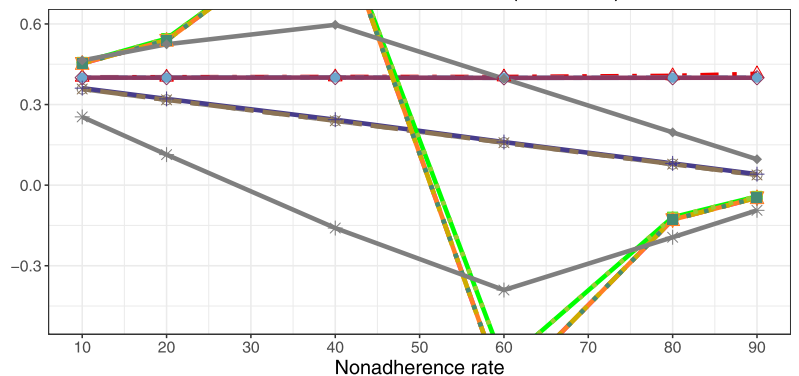

Fig. 24 Mean risk difference (RD) versus the nonadherence rate for DAG 3. Here, violation indicates the violation of the exclusion restriction assumption. The grey lines are the lower and upper bound of the NPCB method. The ITT and baseline-adjusted ITT; naive and both-stages adjusted 2SLS and 2SRI superimposed on each other. Abbreviations: RD: risk difference, ITT: intention-to-treat, PP: per-protocol, AT: as-treated, IP-weighted PP: inverse probability-weighted per-protocol, 2SLS: two-stage least square, 2SRI: two-stage residual inclusion, NPCB: nonparametric causal bound 


\section{Appendix DAGs for sensitivity analysis}

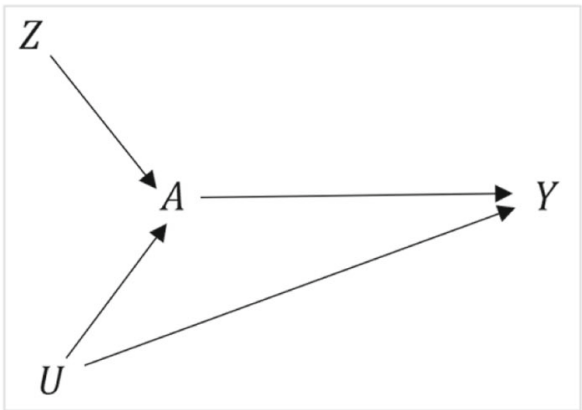

A) Appendix DAG 1

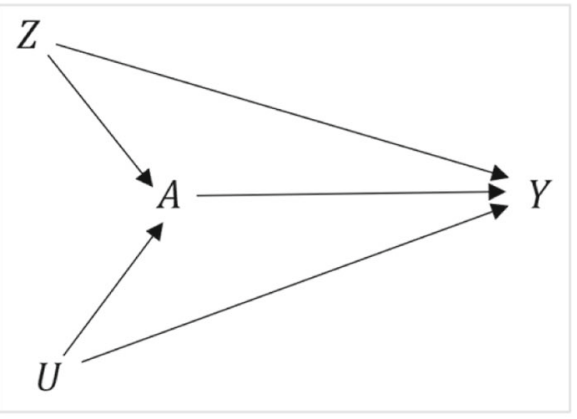

B) Appendix DAG 2

Fig. 25 Two simplified versions of causal diagrams representing the simulation mechanisms considered in this study. Appendix DAG 1 is simplified versions of DAG 2 in the main text (unmeasured confounding), and Appendix DAG 2 is the simplified versions of DAG 3 in the main text (exclusion restriction violated). Here, $Z$ is the randomization variable, $A$ is the treatment, $U$ is unmeasured confounders, and $Y$ is the outcome

Results for sensitivity analyses when exclusion restriction satisfied but unmeasured confounding present (Appendix DAG 1)

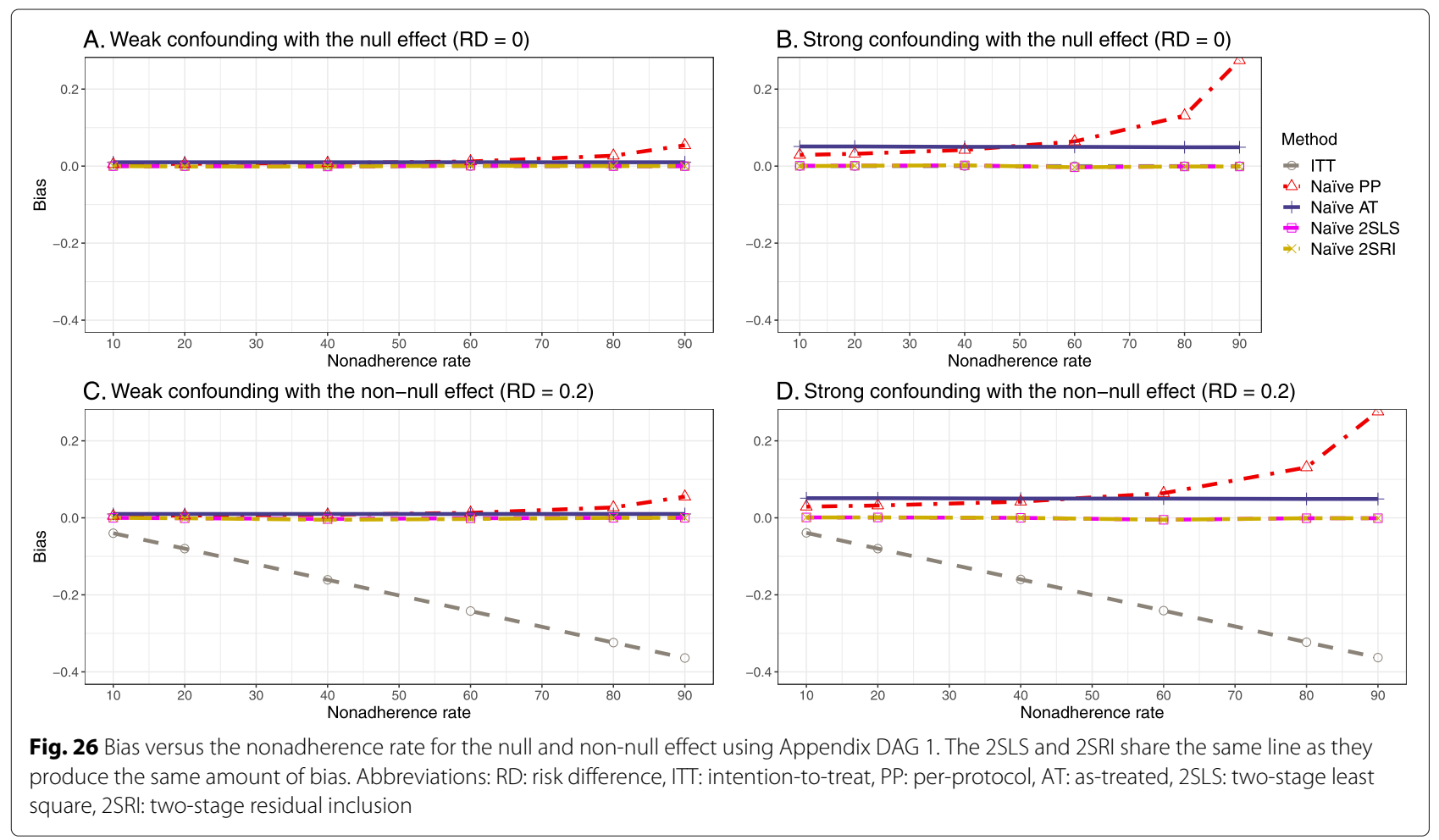



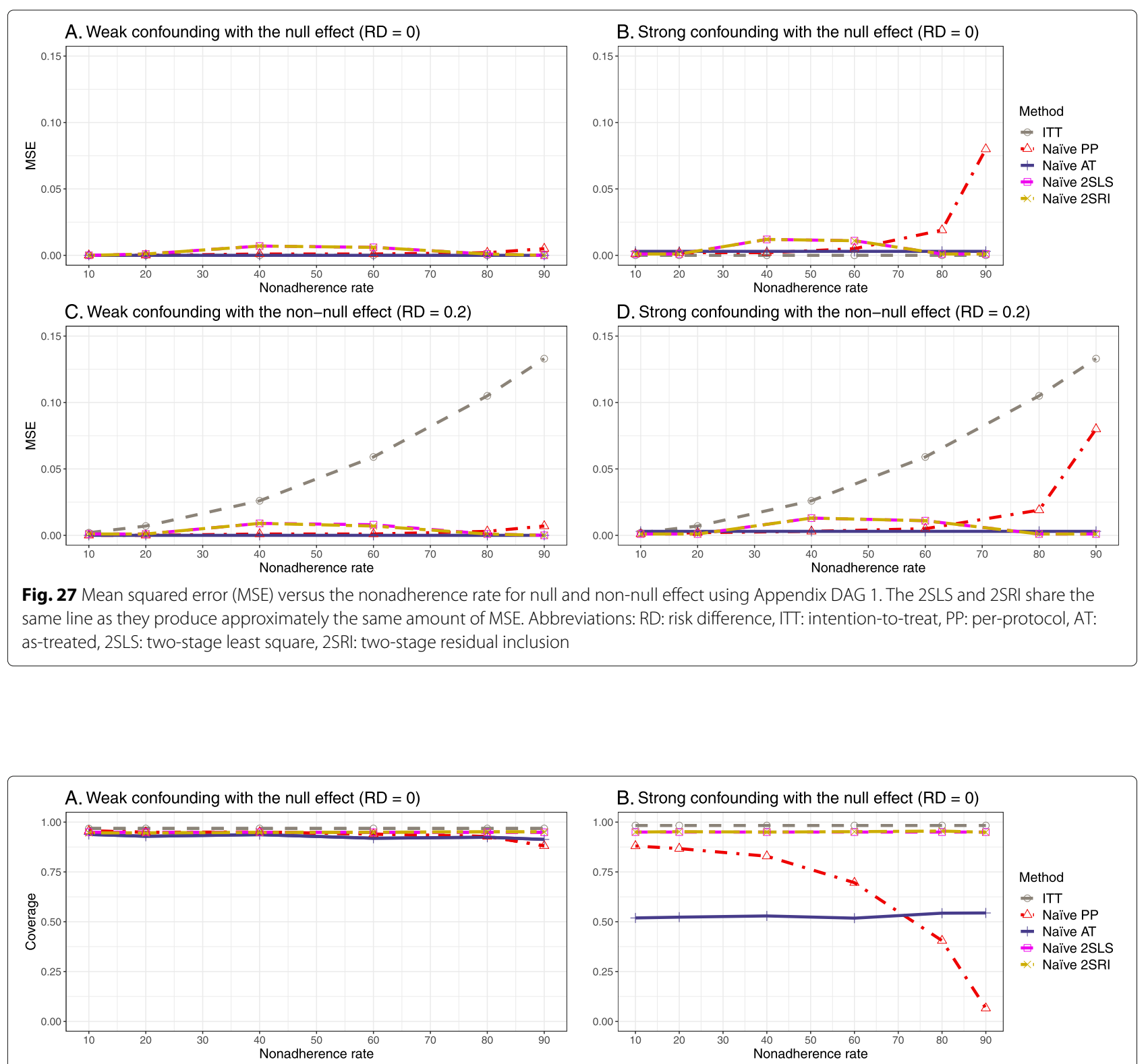

C. Weak confounding with the non-null effect $(R D=0.2)$

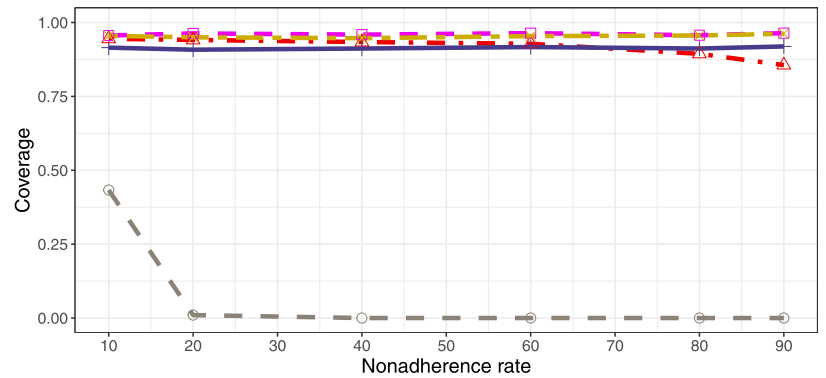

D. Strong confounding with the non-null effect $(\mathrm{RD}=0.2)$

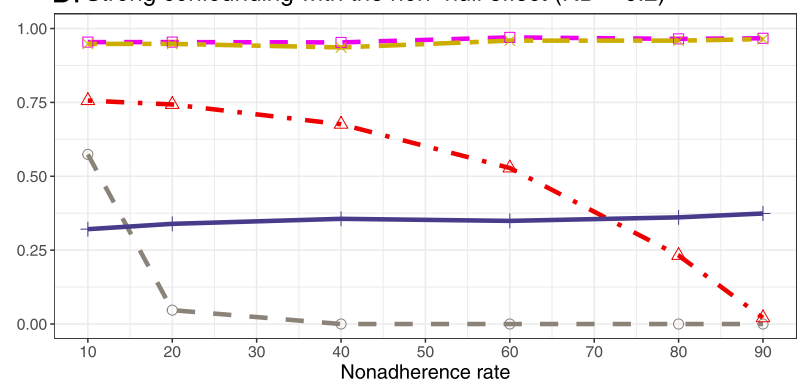

Fig. 2895 percentage coverage probability versus the nonadherence rate for the null and non-null effect using Appendix DAG 1. The 2SLS and 2SRI share the same line as they produce approximately the same coverage probability. Abbreviations: RD: risk difference, ITT: intention-to-treat, PP: per-protocol, AT: as-treated, 2SLS: two-stage least square, 2SRI: two-stage residual inclusion 


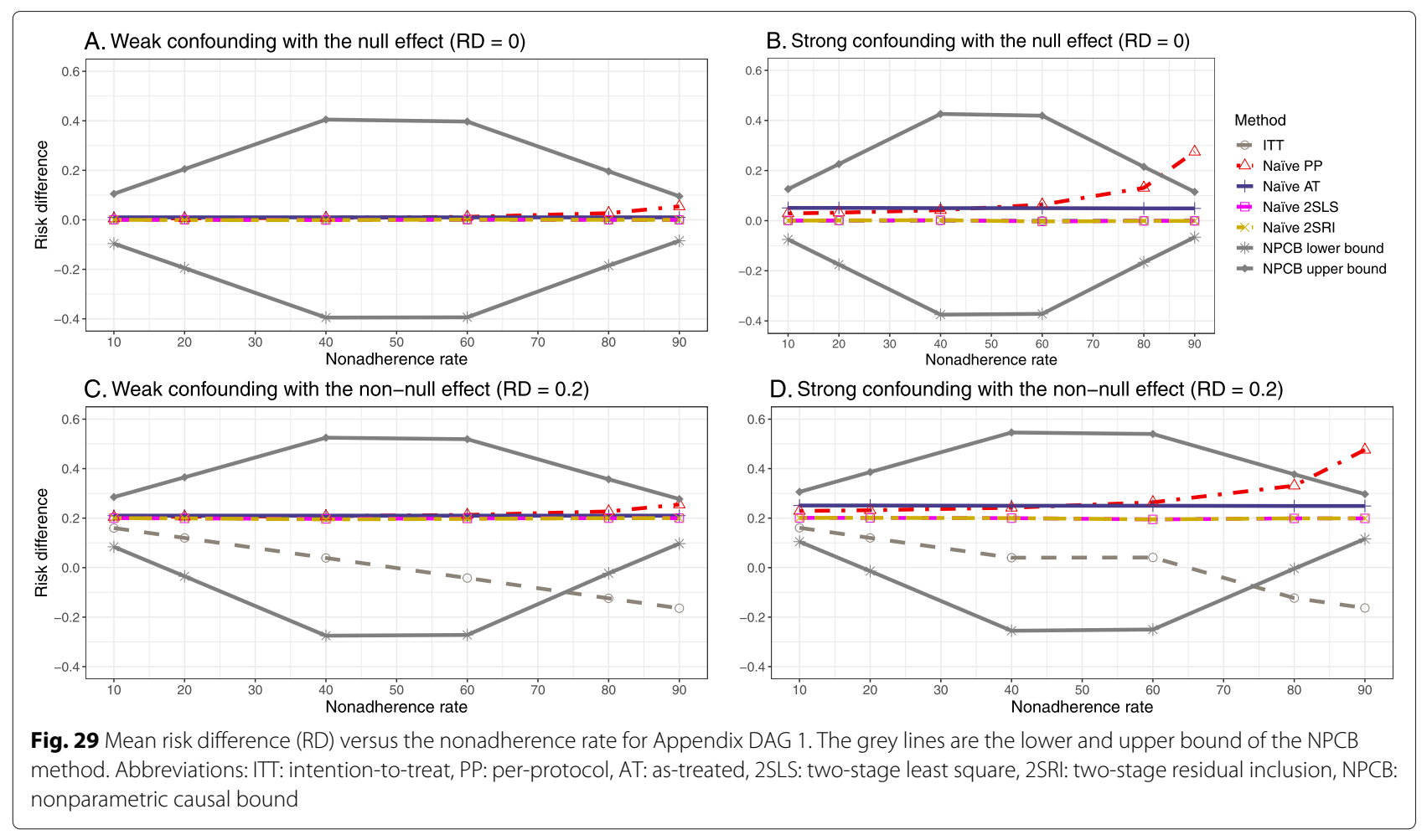

Results for sensitivity analyses when exclusion restriction violated (Appendix DAG 2)

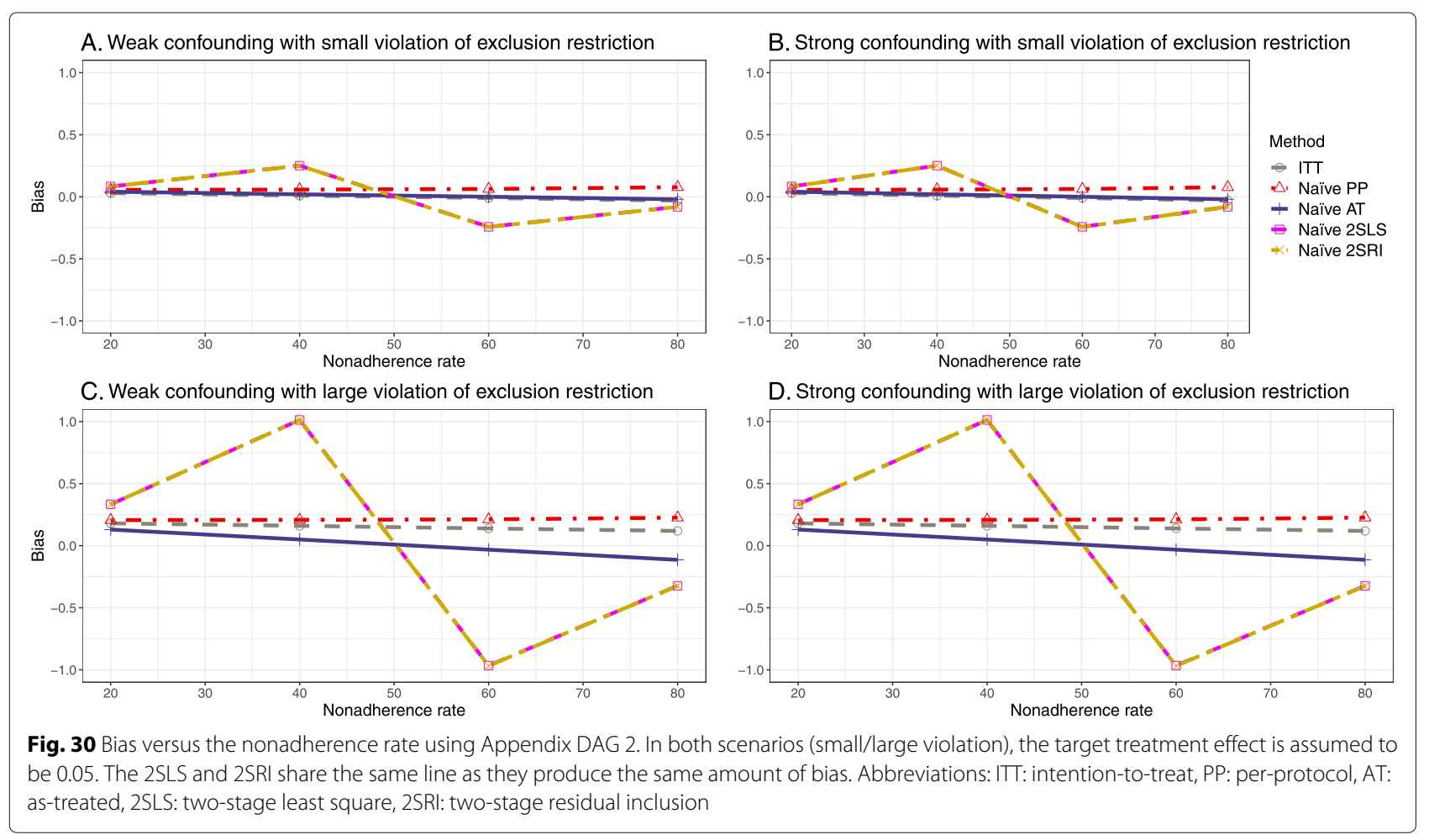




\section{Results for sensitivity analyses with 500 sample size}
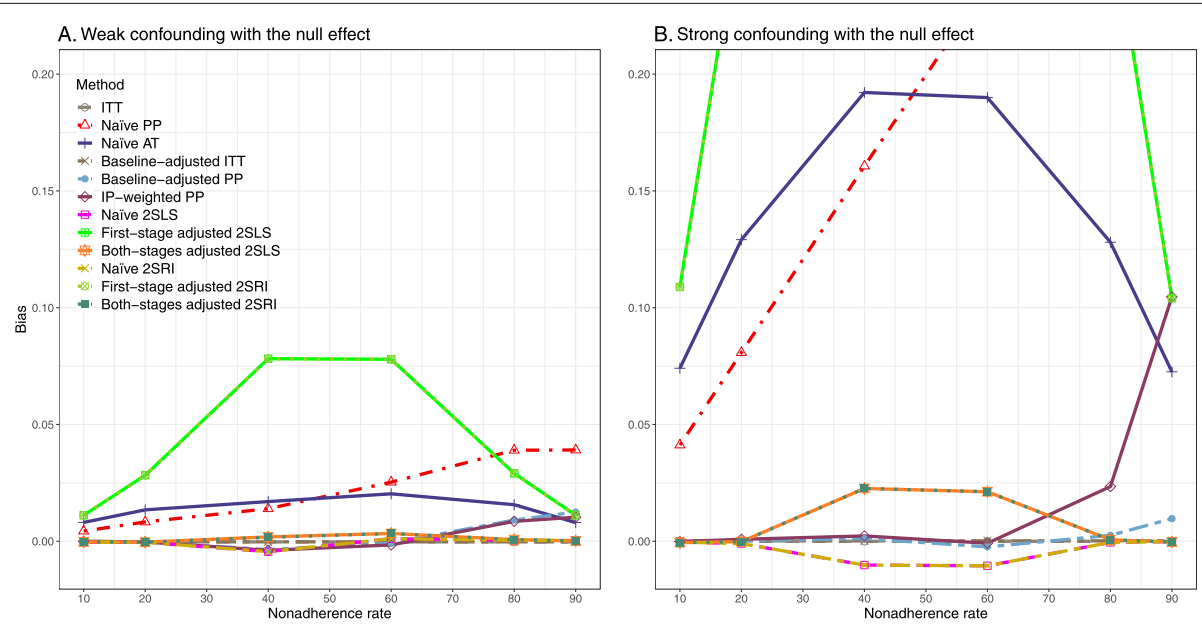

Fig. 31 Bias versus the nonadherence rate using DAG 1 for 500 samples. The naive 2SLS and 2SRI share the same line, the first stage adjusted 2SLS and 2SRI share the same line, and both-stages adjusted 2SLS and 2SRI share the same line as they produce the same amount of bias. The first stage adjusted 2SLS and 2SRI, and naive PP produce larger than 0.20 bias for strong unmeasured confounders so that the bias is out of the bound. Abbreviations: ITT: intention-to-treat, PP: per-protocol, AT: as-treated, IP-weighted PP: inverse probability-weighted per-protocol, 2SLS: two-stage least square, 2SRI: two-stage residual inclusion
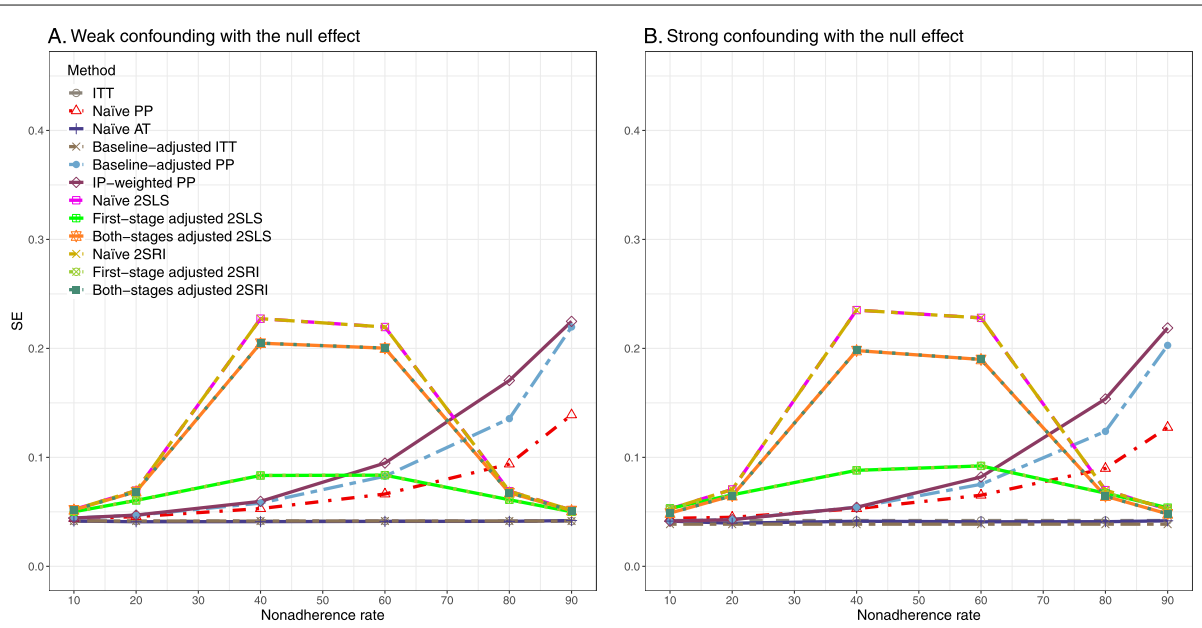

Fig. 32 Standard error (SE) versus the nonadherence rate using DAG 1 for 500 samples. The naïve 2SLS and 2SRI share the same line, the first stage adjusted 2SLS and 2SRI share the same line, and both-stages adjusted 2SLS and 2SRI share the same line as they produce approximately the same SE. Abbreviations: ITT: intention-to-treat, PP: per-protocol, AT: as-treated, IP-weighted PP: inverse probability-weighted per-protocol, 2SLS: two-stage least square, 2SRI: two-stage residual inclusion 

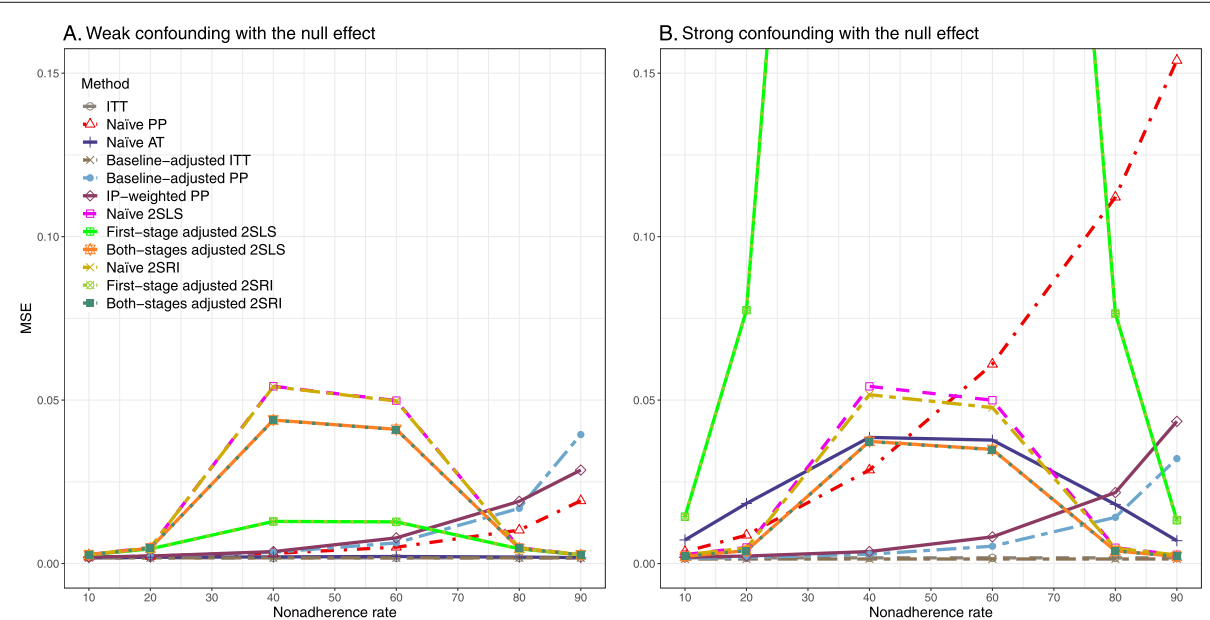

Fig. 33 Mean squared error (MSE) versus the nonadherence rate using DAG 1 for 500 samples. The naïve 2SLS and 2SRI share the same line, the first stage adjusted 2SLS and 2SRI share the same line, and both-stages adjusted 2SLS and 2SRI share the same line as they produce approximately the same MSE. The first stage adjusted 2SLS and 2SRI, and naïve PP produce MSEs larger than 0.15 for strong unmeasured confounders so that the MSE is out of the bound [0, 0.15]. Abbreviations: ITT: intention-to-treat, PP: per-protocol, AT: as-treated, IP-weighted PP: inverse probability-weighted per-protocol, 2SLS: two-stage least square, 2SRI: two-stage residual inclusion
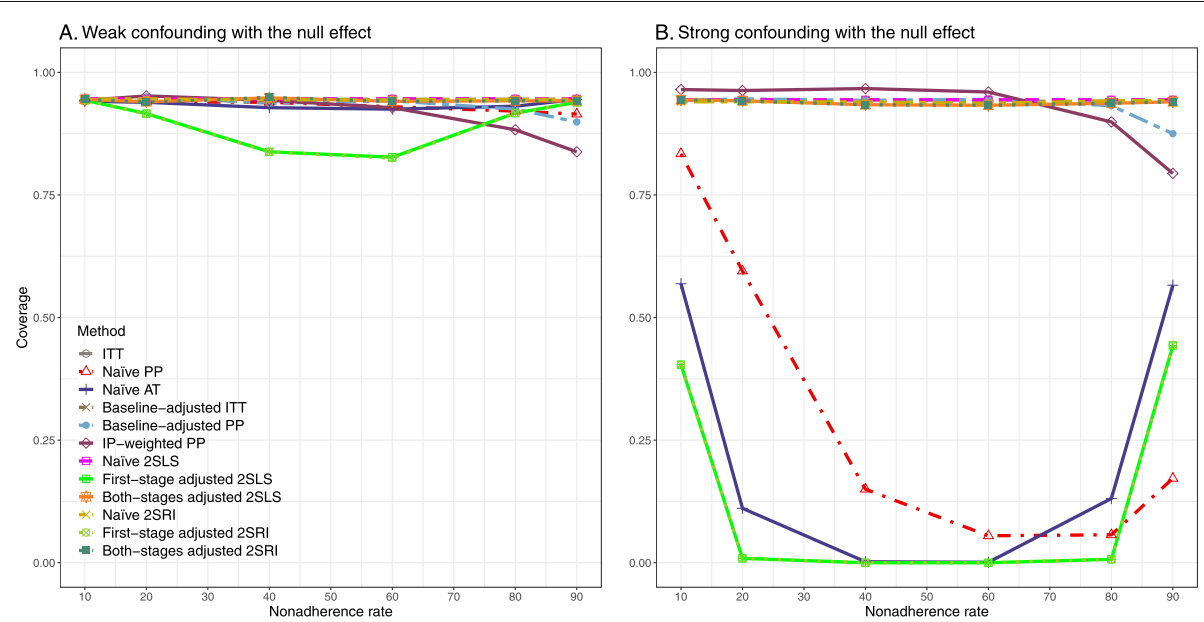

Fig. 3495 percentage coverage probability versus the nonadherence rate using DAG 1 for 500 samples. The naïve 2SLS and 2SRI share the same line, the first-stage adjusted 2SLS and 2SRI share the same line, and both-stages adjusted 2SLS and 2SRI share the same line as they produce approximately the same coverage probability. Abbreviations: ITT: intention-to-treat, PP: per-protocol, AT: as-treated, IP-weighted PP: inverse probability-weighted per-protocol, 2SLS: two-stage least square, 2SRI: two-stage residual inclusion 

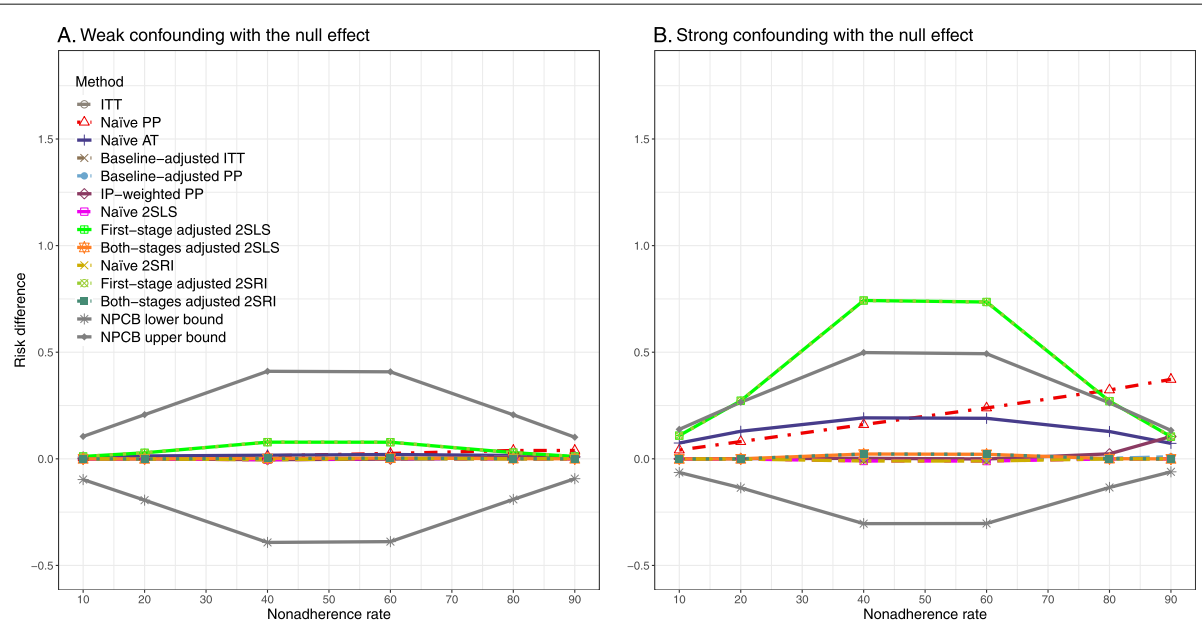

Fig. 35 Mean risk difference (RD) versus the nonadherence rate using DAG 1 for 500 samples. The grey lines are the lower and upper bound of the NPCB method. The naïve 2SLS and 2SRI share the same line, the first stage adjusted 2SLS and 2SRI share the same line, and both-stages adjusted 2SLS and 2SRI share the same line as they produce the same RD estimate. Abbreviations: ITT: intention-to-treat, PP: per-protocol, AT: as-treated, IP-weighted PP: inverse probability-weighted per-protocol, 2SLS: two-stage least square, 2SRI: two-stage residual inclusion, NPCB: nonparametric causal bound

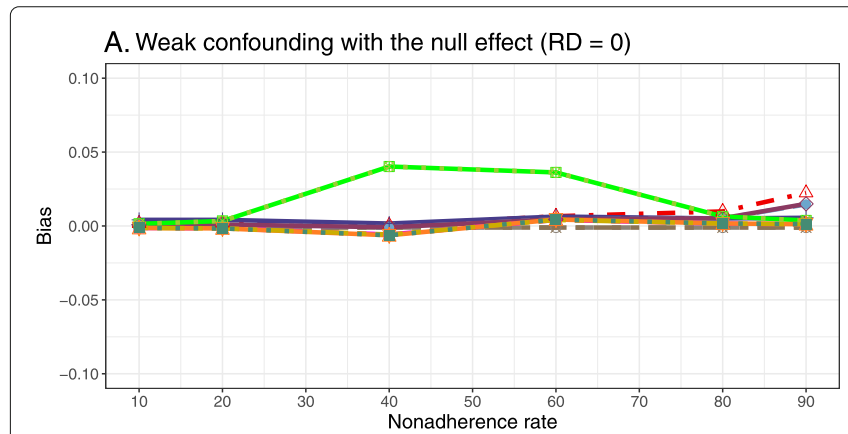

C. Weak confounding with the non-null effect $(R D=0.2)$

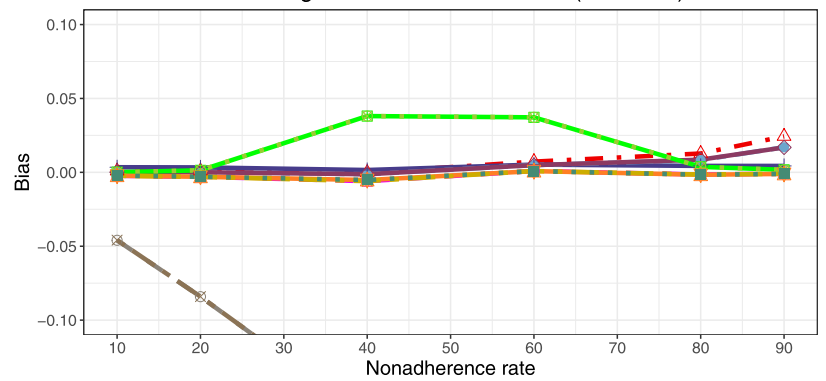

B. Strong confounding with the null effect $(\mathrm{RD}=0)$

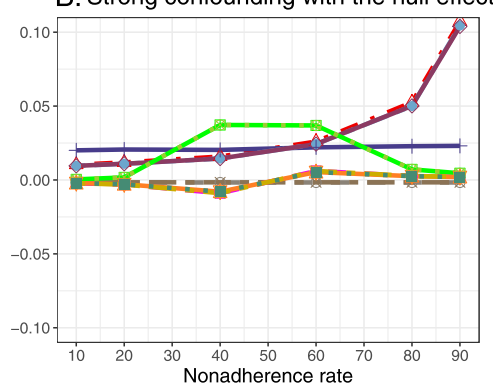

Method

$\because$ ITT

$\triangle$ Naïve PP

+ Naïve AT

$*$ : Baseline-adjusted ITT

$\Rightarrow$ Baseline-adjusted PP

$\Leftrightarrow \mathrm{IP}$-weighted PP

- Naïve 2SLS

First-stage adjusted 2SLS

Both-stages adjusted 2SLS

$*$ Both-stages
$*$. Naïve 2SRI

Wir. First-stage adjusted 2SRI

-1.' Both-stages adjusted 2SRI

D. Strong confounding with the non-null effect $(R D=0.2)$

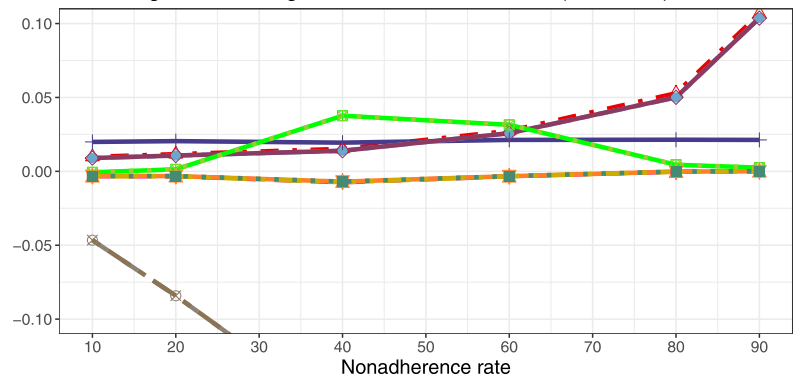

Fig. 36 Bias versus the nonadherence rate using DAG 2 for 500 samples. The naïve and both-stages adjusted 2SLS and 2SRI share the same line, and the first stage adjusted 2SLS and 2SRI share the same line as they produce the same amount of bias. Abbreviations: RD: risk difference, ITT: intention-to-treat, PP: per-protocol, AT: as-treated, IP-weighted PP: inverse probability-weighted per-protocol, 2SLS: two-stage least square, 2SRI: two-stage residual inclusion 

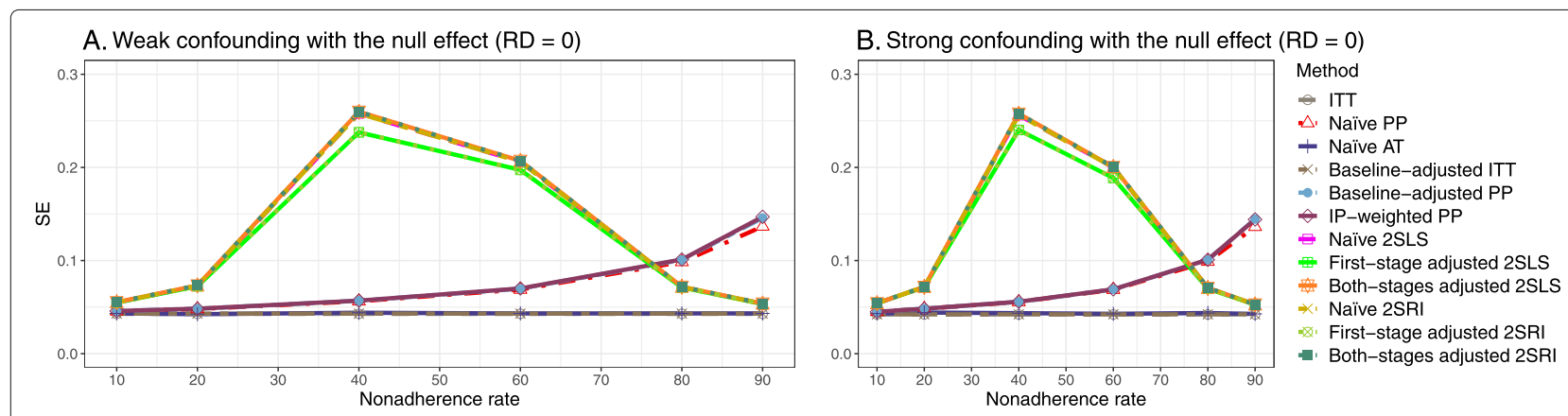

C. Weak confounding with the non-null effect $(\mathrm{RD}=0.2)$

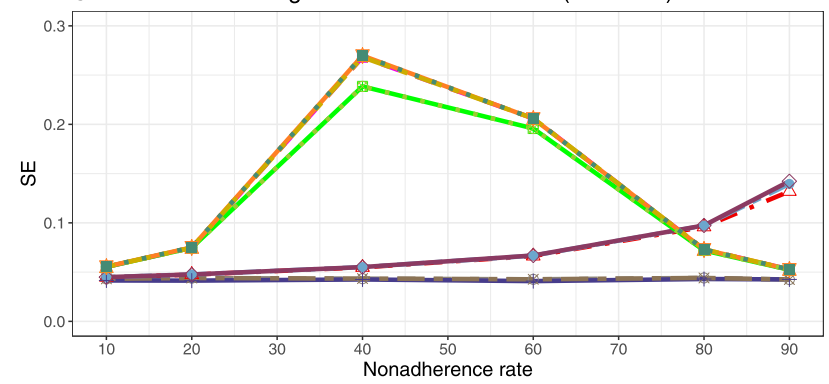

D. Strong confounding with the non-null effect $(\mathrm{RD}=0.2)$

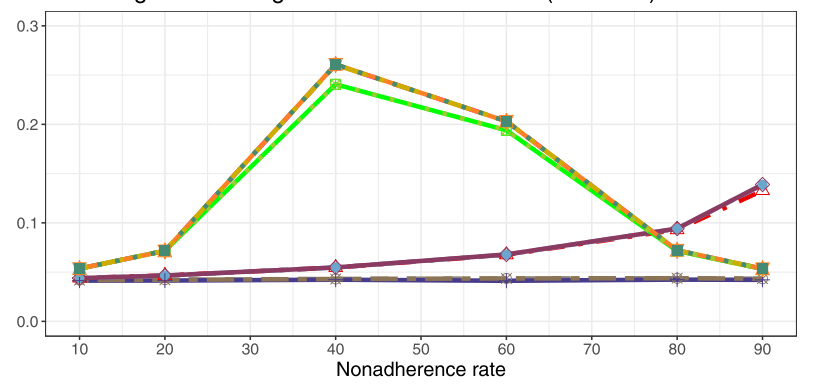

Fig. 37 Standard error (SE) versus the nonadherence rate using DAG 2 for 500 samples. The naïve and both-stages adjusted 2SLS and 2SRI share the same line, and the first stage adjusted 2SLS and 2SRI share the same line as they produce approximately the same SE. The PP methods also share approximately the same line. Abbreviations: RD: risk difference, ITT: intention-to-treat, PP: per-protocol, AT: as-treated, IP-weighted PP: inverse probability-weighted per-protocol, 2SLS: two-stage least square, 2SRI: two-stage residual inclusion

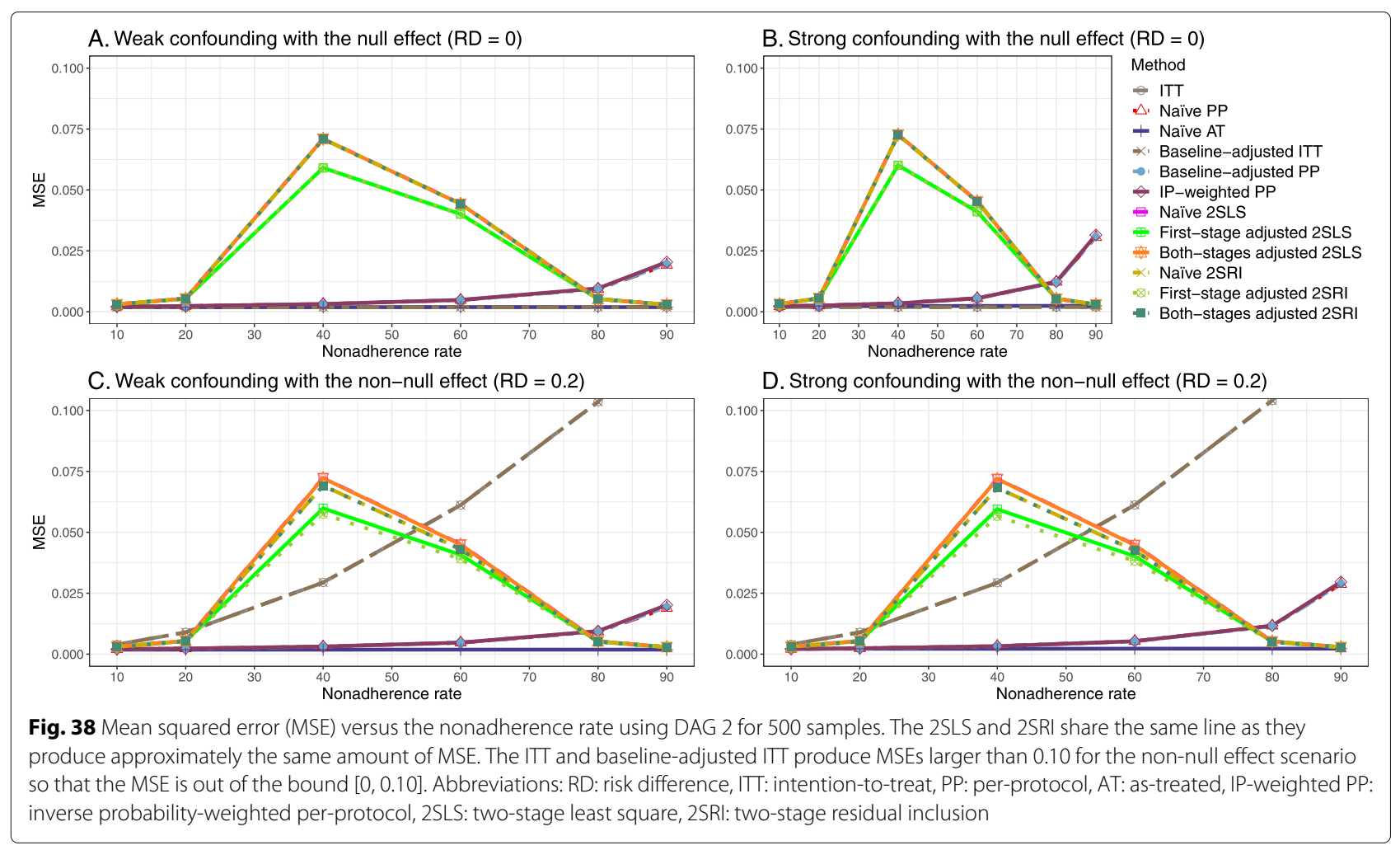




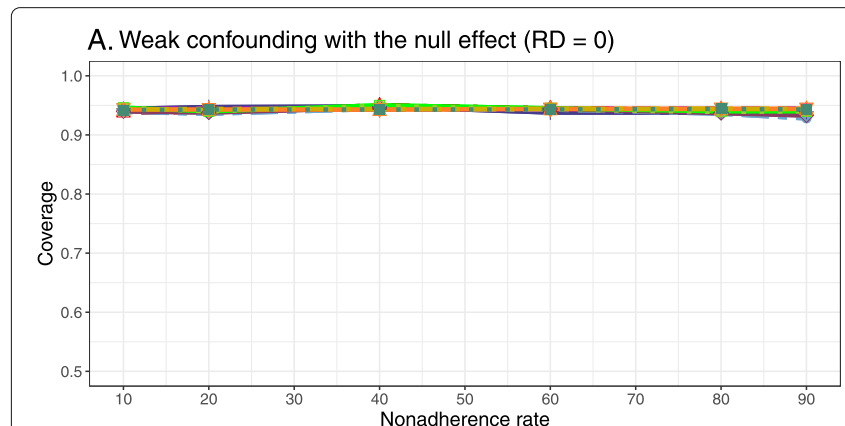

C. Weak confounding with the non-null effect $(R D=0.2)$

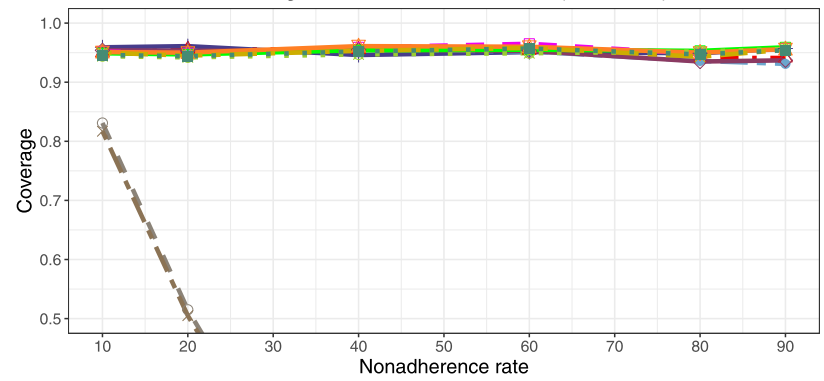

B. Strong confounding with the null effect $(\mathrm{RD}=0)$

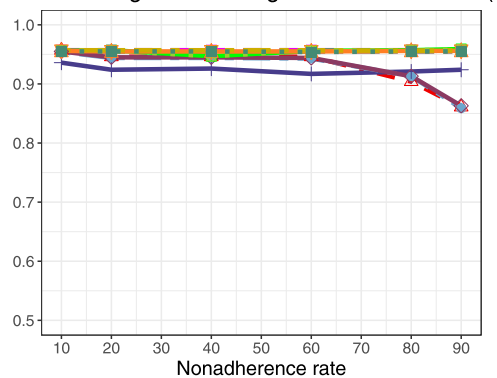

Method

$\Leftrightarrow$ ITT

$\triangle$ Naïve PP

+ Naïve AT

$\forall *$ Baseline-adjusted ITT

$\Rightarrow$ Baseline-adjusted PP

$\approx \mathrm{IP}$-weighted PP

־ Naïve 2SLS

- First-stage adjusted 2SLS

Both-stages adjusted 2SLS

*`. Naïve 2SRI

₹. First-stage adjusted 2SRI

- Both-stages adjusted 2SRI

D. Strong confounding with the non-null effect $(R D=0.2)$

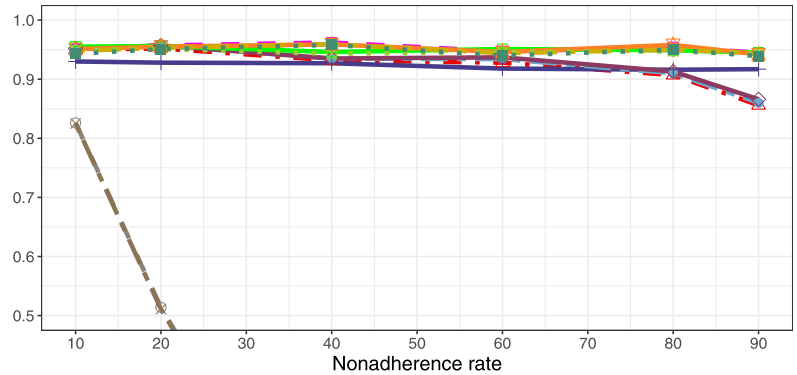

Fig. 3995 percentage coverage probability versus the nonadherence rate for using DAG 2 for 500 samples. The naive and both-stages adjusted 2SLS and 2SRI share the same line as they produce approximately the same coverage probability. The coverage probability is out of bound for the ITT methods with the non-null effect. Abbreviations: RD: risk difference, ITT: intention-to-treat, PP: per-protocol, AT: as-treated, IP-weighted PP: inverse probability-weighted per-protocol, 2SLS: two-stage least square, 2SRI: two-stage residual inclusion

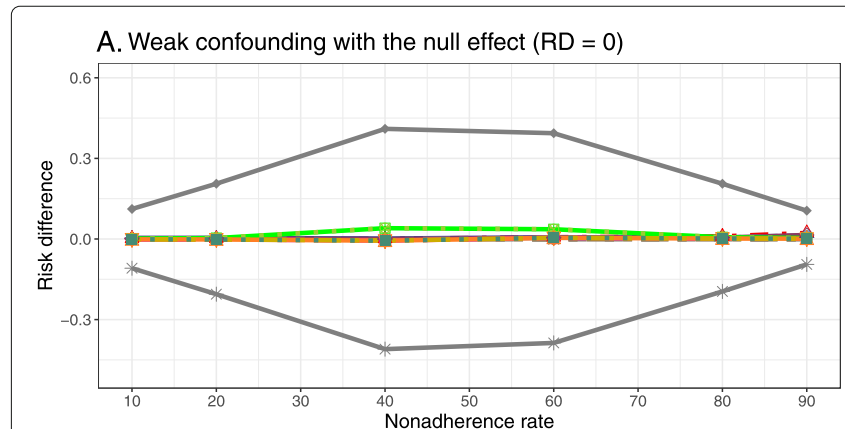

C. Weak confounding with the non-null effect $(\mathrm{RD}=0.2)$

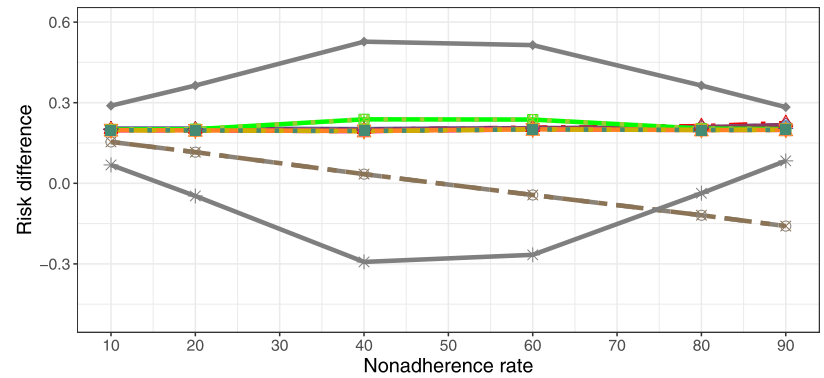

\section{B. Strong confounding with the null effect $(\mathrm{RD}=$ Qhethod}

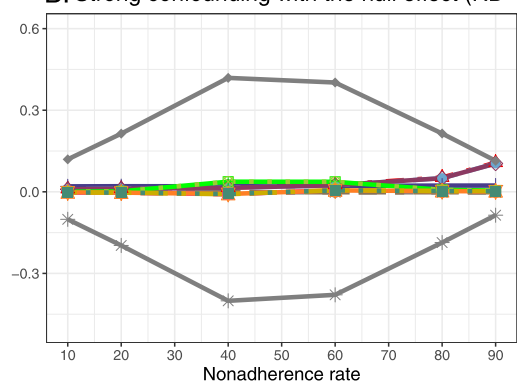

$\because \mathrm{ITT}$

$\triangle$ Naïve PP

+ Naĩve AT

* Baseline-adjusted ITT

$\rightarrow$ Baseline-adjusted PP

$\Leftrightarrow$ IP-weighted PP

- Naïve 2SLS

- First-stage adjusted 2SLS

Both-stages adjusted 2SLS

\#< Naïve 2SRI

*8. First-stage adjusted 2SRI

- Both-stages adjusted 2SRI

* NPCB lower bound

- NPCB upper bound

D. Strong confounding with the non-null effect $(\mathrm{RD}=0.2)$

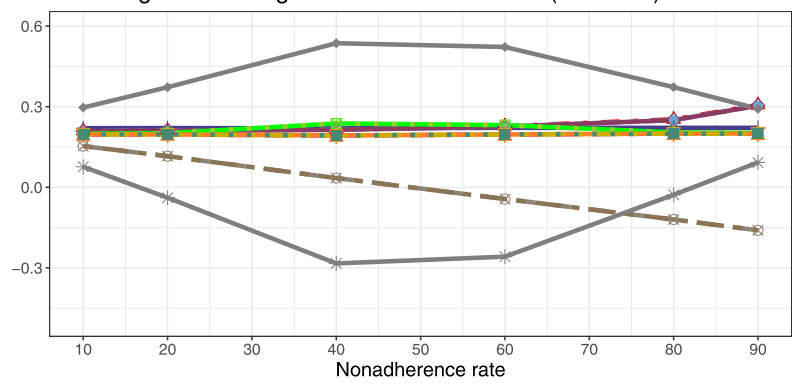

Fig. 40 Mean risk difference (RD) versus the nonadherence rate for using DAG 2 for 500 samples. The grey lines are the lower and upper bound of the NPCB method. The ITT and baseline-adjusted ITT superimposed on each other. Abbreviations: RD: risk difference, ITT: intention-to-treat, PP: per-protocol, AT: as-treated, IP-weighted PP: inverse probability-weighted per-protocol, 2SLS: two-stage least square, 2SRI: two-stage residual inclusion, NPCB: nonparametric causal bound 


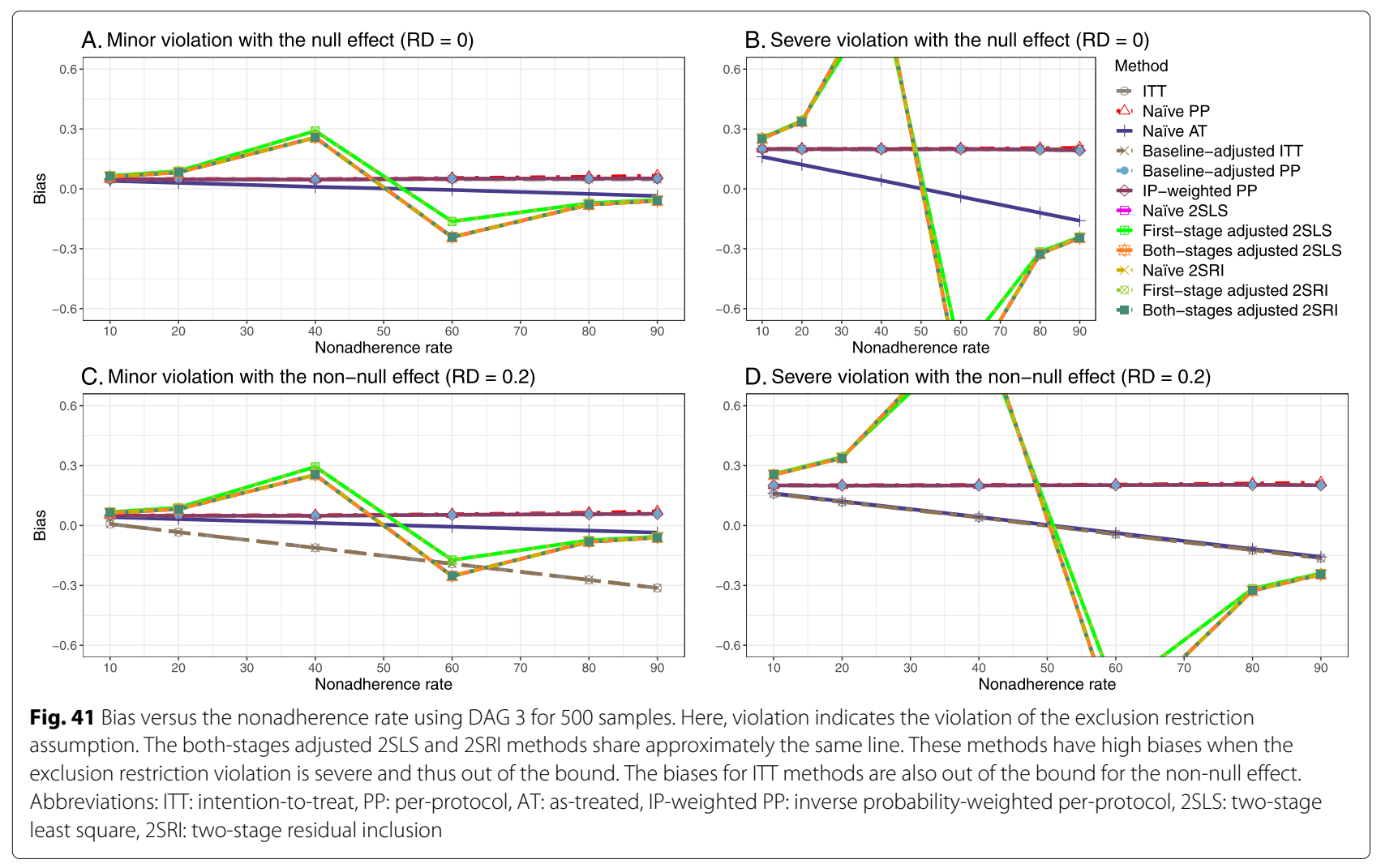

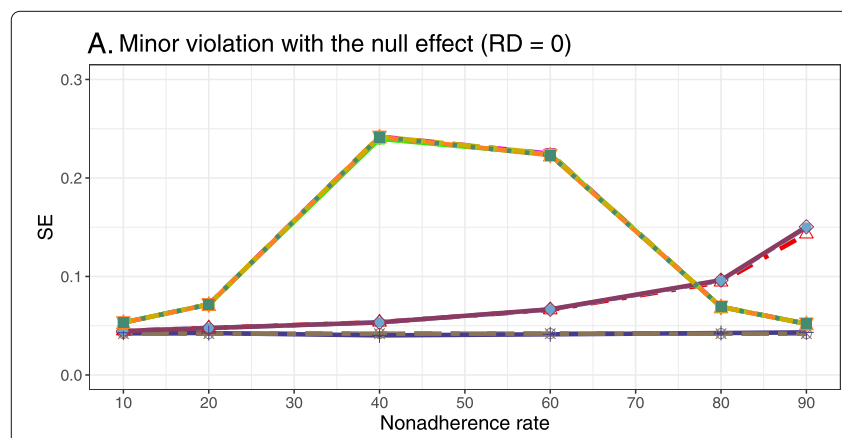

C. Minor violation with the non-null effect $(R D=0.2)$

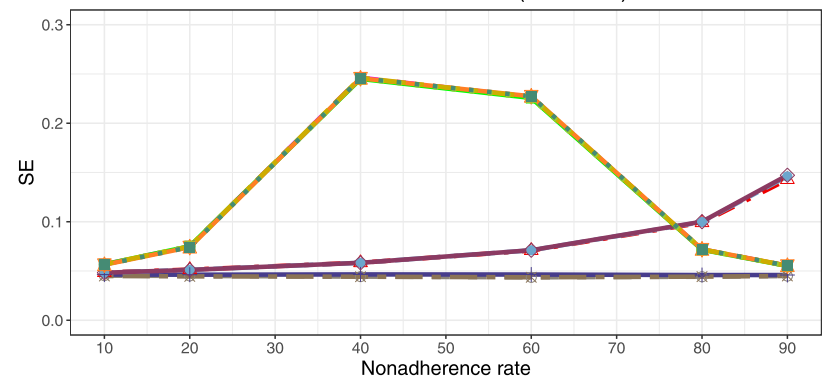

\section{B. Severe violation with the null effect $(\mathrm{RD}=0)$}

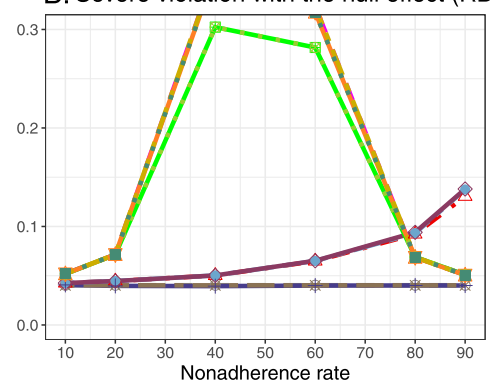

Method

$\because$ ITT

$\triangle$ Naïve PP

- Naîve AT

*: Baseline-adjusted ITT

$\rightarrow$ Baseline-adjusted PP

$\Leftrightarrow$ IP-weighted PP

$\because$ Naîve $2 S L S$

\# First-stage adjusted 2SLS

Both-stages adjusted 2SLS

*. Naîve 2SRI

w. First-stage adjusted 2SRI

* Both-stages adjusted 2SRI

\section{Severe violation with the non-null effect $(R D=0.2)$}

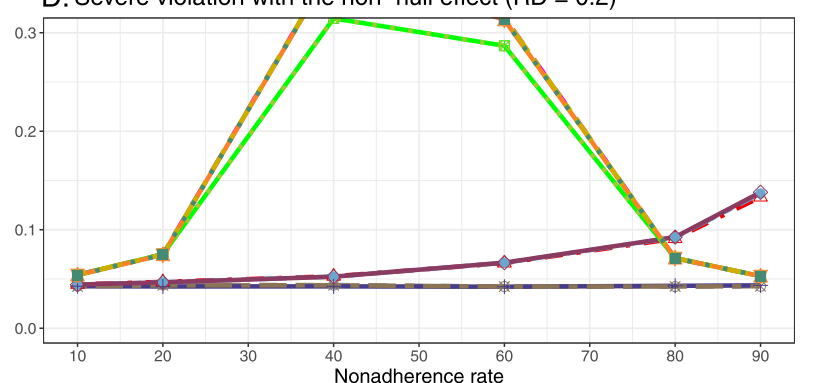

Fig. 42 Standard error (SE) versus the nonadherence rate using DAG 3 for 500 samples. Here, violation indicates the violation of the exclusion restriction assumption. Abbreviations: ITT: intention-to-treat, PP: per-protocol, AT: as-treated, IP-weighted PP: inverse probability-weighted per-protocol, 2SLS: two-stage least square, 2SRI: two-stage residual inclusion 

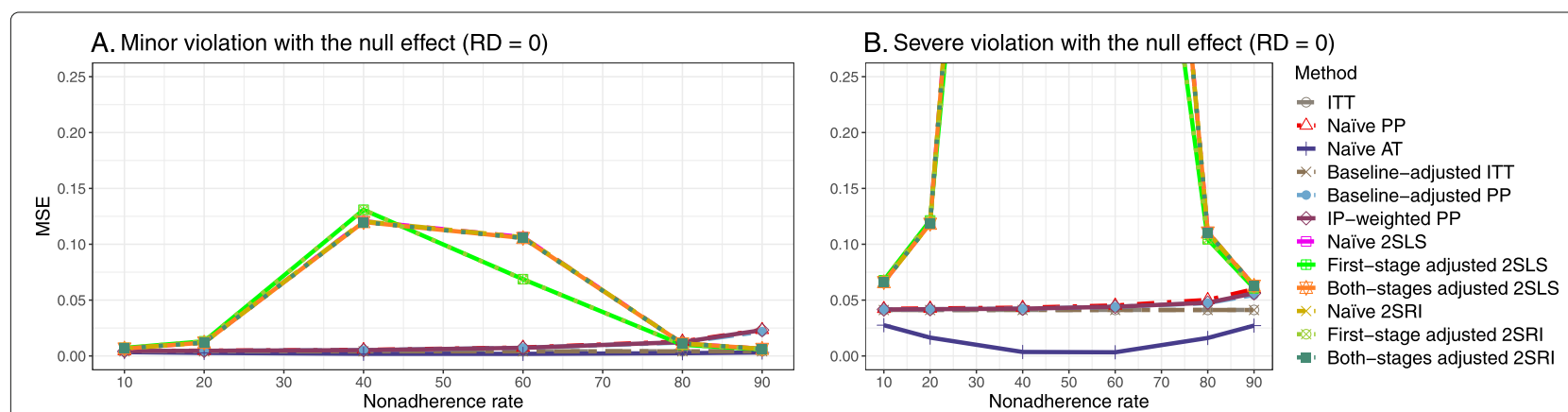

C. Minor violation with the non-null effect $(\mathrm{RD}=0.2)$

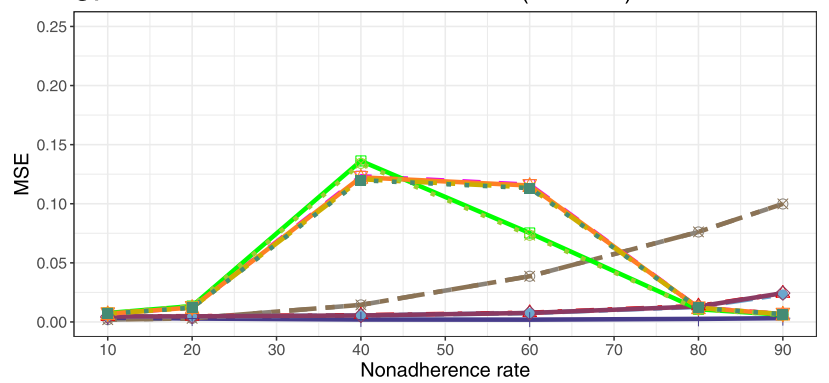

D. Severe violation with the non-null effect $(R D=0.2)$

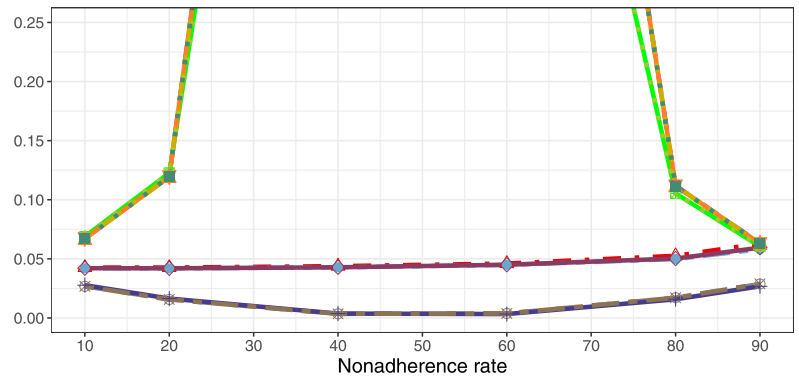

Fig. 43 Mean squared error (MSE) versus the nonadherence rate using DAG 3 for 500 samples. Here, violation indicates the violation of the exclusion restriction assumption. Abbreviations: ITT: intention-to-treat, PP: per-protocol, AT: as-treated, IP-weighted PP: inverse probability-weighted per-protocol, 2SLS: two-stage least square, 2SRI: two-stage residual inclusion

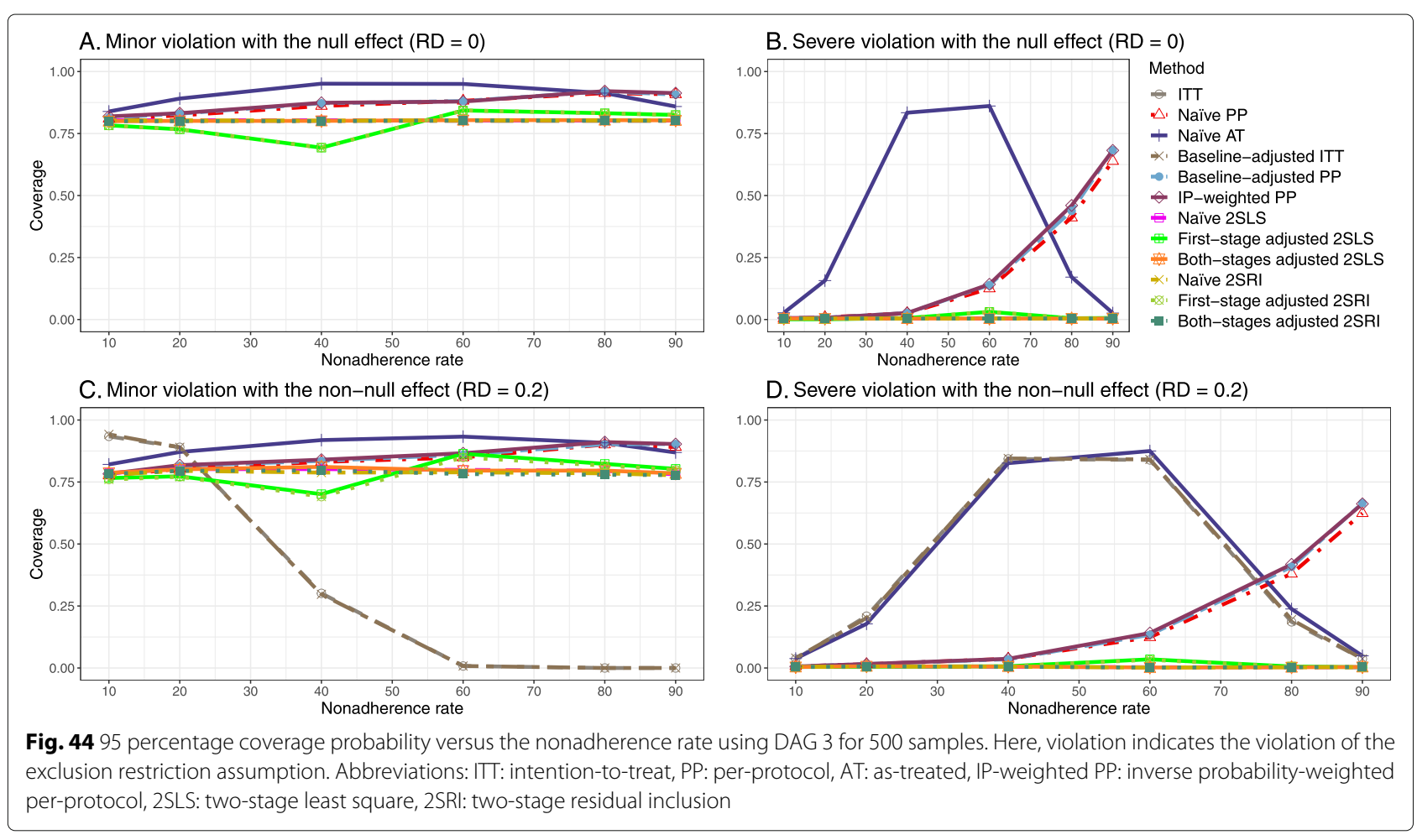




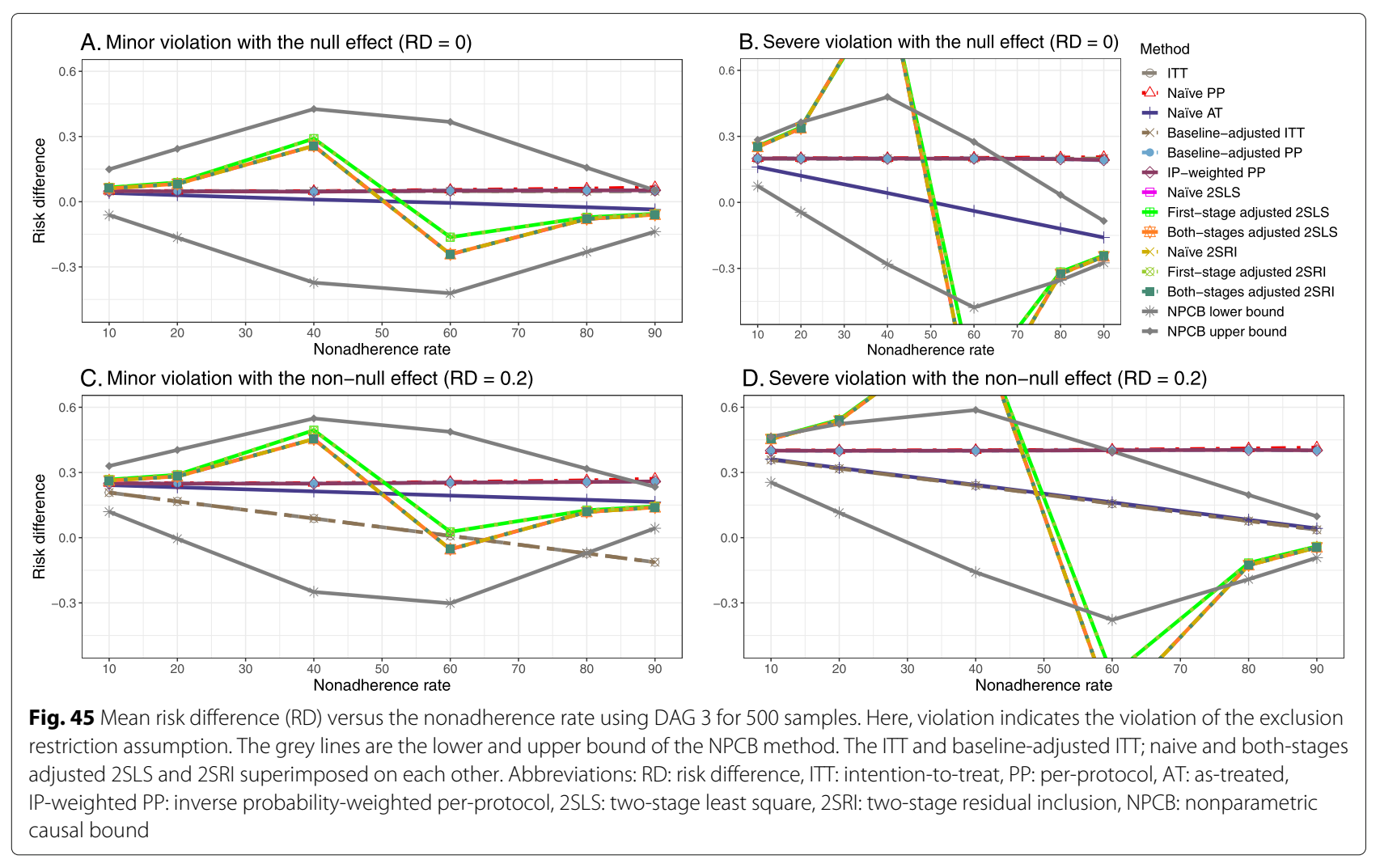

\section{Present study's recommendation}

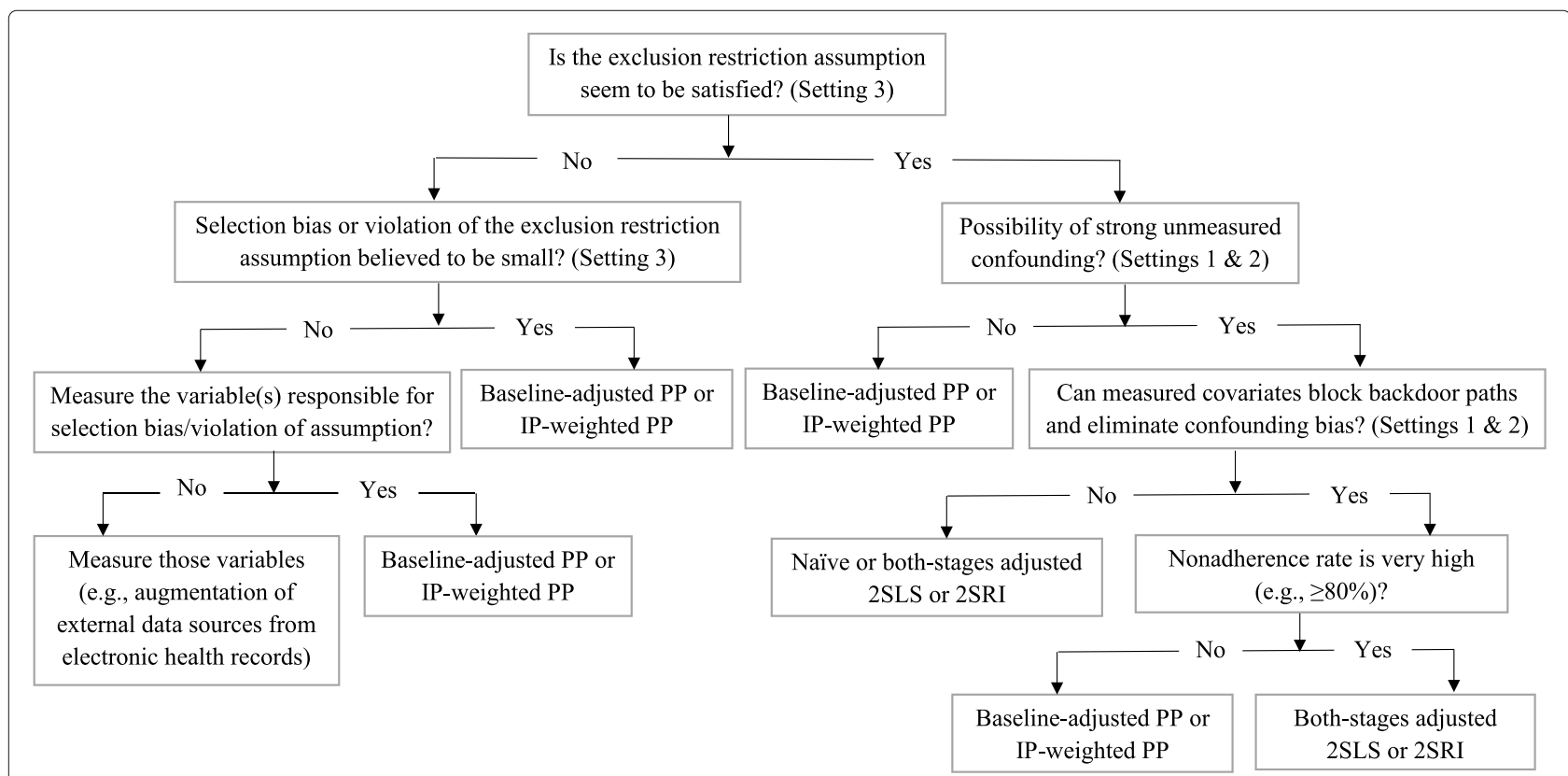

Fig. 46 Flow chart showing the conclusion or recommendation from the present study. Abbreviations: PP: per-protocol, IP-weighted PP: inverse probability-weighted per-protocol, 2SLS: two-stage least square, 2SRI: two-stage residual inclusion 


\section{Appendix D}

This section is for the parameterization of each three data-generating processes in this study. Appendix D Tables 5-7 show the parameters for the simulation settings 1-3 outlined in DAGs 1-3, respectively. In each table, we have the same set of parameters: $\alpha_{0}$ determines the nonadherence rate, $\alpha_{1}$ is the coefficient for $Z$ - $A$ association, $\alpha_{2}$ is the coefficient for $L_{1}-A$ association, $\alpha_{3}$ is the coefficient for $L_{2}-A$ association, and $\alpha_{4}$ is the coefficient for $U-A$ association. Also, $\theta_{0}$ determines the event rate, $\theta_{1}$ is treatment effect of interest (i.e., the coefficient for $A-Y$ association), $\theta_{2}$ is the coefficient for $L_{1}-Y$ association, $\theta_{3}$ is the coefficient for $L_{2}-Y$ association, $\theta_{4}$ is the coefficient for $U-Y$ association, and $\theta_{5}$ determines the $Z-Y$ association.

Table 5 Parameterization of simulation for data generating process for simulation setting 1 outlines in DAG 1 (exclusion restriction satisfied and no unmeasured confounding)

\begin{tabular}{|c|c|c|c|c|c|c|c|c|c|c|c|c|c|}
\hline$\#$ & Arm & $\alpha_{0}$ & $\alpha_{1}$ & $\alpha_{2}$ & $\alpha_{3}$ & $\alpha_{4}$ & Nonadherence & $\theta_{0}$ & $\theta_{1}$ & $\theta_{2}$ & $\theta_{3}$ & $\theta_{4}$ & $\theta_{5}$ \\
\hline \multirow{2}{*}{1} & $Z=1$ & 0.72 & 0.6 & 0.4 & 0.35 & 0 & 10 & -1 & 0 & 0 & 0 & 0.5 & 0 \\
\hline & $Z=0$ & -4.06 & 0.6 & 0.4 & 0.35 & 0 & 10 & -1 & 0 & 0 & 0 & 0.5 & 0 \\
\hline \multirow{2}{*}{2} & $Z=1$ & -0.23 & 0.6 & 0.4 & 0.35 & 0 & 20 & -1 & 0 & 0 & 0 & 0.5 & 0 \\
\hline & $Z=0$ & -3.14 & 0.6 & 0.4 & 0.35 & 0 & 20 & -1 & 0 & 0 & 0 & 0.5 & 0 \\
\hline \multirow{2}{*}{3} & $Z=1$ & -1.47 & 0.6 & 0.4 & 0.35 & 0 & 40 & -1 & 0 & 0 & 0 & 0.5 & 0 \\
\hline & $Z=0$ & -1.92 & 0.6 & 0.4 & 0.35 & 0 & 40 & -1 & 0 & 0 & 0 & 0.5 & 0 \\
\hline \multirow{2}{*}{4} & $Z=1$ & -2.52 & 0.6 & 0.4 & 0.35 & 0 & 60 & -1 & 0 & 0 & 0 & 0.5 & 0 \\
\hline & $Z=0$ & -0.85 & 0.6 & 0.4 & 0.35 & 0 & 60 & -1 & 0 & 0 & 0 & 0.5 & 0 \\
\hline \multirow{2}{*}{5} & $Z=1$ & -3.76 & 0.6 & 0.4 & 0.35 & 0 & 80 & -1 & 0 & 0 & 0 & 0.5 & 0 \\
\hline & $Z=0$ & 0.39 & 0.6 & 0.4 & 0.35 & 0 & 80 & -1 & 0 & 0 & 0 & 0.5 & 0 \\
\hline \multirow{2}{*}{6} & $Z=1$ & -4.72 & 0.6 & 0.4 & 0.35 & 0 & 90 & -1 & 0 & 0 & 0 & 0.5 & 0 \\
\hline & $Z=0$ & 1.35 & 0.6 & 0.4 & 0.35 & 0 & 90 & -1 & 0 & 0 & 0 & 0.5 & 0 \\
\hline \multirow{2}{*}{7} & $Z=1$ & 0.72 & 0.6 & 0.4 & 0.35 & 0 & 10 & -5.5 & 0 & 0 & 0 & 8 & 0 \\
\hline & $Z=0$ & -4.06 & 0.6 & 0.4 & 0.35 & 0 & 10 & -5.5 & 0 & 0 & 0 & 8 & 0 \\
\hline \multirow{2}{*}{8} & $Z=1$ & -0.23 & 0.6 & 0.4 & 0.35 & 0 & 20 & -5.5 & 0 & 0 & 0 & 8 & 0 \\
\hline & $Z=0$ & -3.14 & 0.6 & 0.4 & 0.35 & 0 & 20 & -5.5 & 0 & 0 & 0 & 8 & 0 \\
\hline \multirow{2}{*}{9} & $Z=1$ & -1.47 & 0.6 & 0.4 & 0.35 & 0 & 40 & -5.5 & 0 & 0 & 0 & 8 & 0 \\
\hline & $Z=0$ & -1.92 & 0.6 & 0.4 & 0.35 & 0 & 40 & -5.5 & 0 & 0 & 0 & 8 & 0 \\
\hline \multirow{2}{*}{10} & $Z=1$ & -2.52 & 0.6 & 0.4 & 0.35 & 0 & 60 & -5.5 & 0 & 0 & 0 & 8 & 0 \\
\hline & $Z=0$ & -0.85 & 0.6 & 0.4 & 0.35 & 0 & 60 & -5.5 & 0 & 0 & 0 & 8 & 0 \\
\hline \multirow{2}{*}{11} & $Z=1$ & -3.76 & 0.6 & 0.4 & 0.35 & 0 & 80 & -5.5 & 0 & 0 & 0 & 8 & 0 \\
\hline & $Z=0$ & 0.39 & 0.6 & 0.4 & 0.35 & 0 & 80 & -5.5 & 0 & 0 & 0 & 8 & 0 \\
\hline \multirow{2}{*}{12} & $Z=1$ & -4.72 & 0.6 & 0.4 & 0.35 & 0 & 90 & -5.5 & 0 & 0 & 0 & 8 & 0 \\
\hline & $Z=0$ & 1.35 & 0.6 & 0.4 & 0.35 & 0 & 90 & -5.5 & 0 & 0 & 0 & 8 & 0 \\
\hline
\end{tabular}

Table 6 Parameterization of simulation for data generating process for simulation setting 2 outlines in DAG 2 (Exclusion restriction satisfied, unmeasured confounding present)

\begin{tabular}{|c|c|c|c|c|c|c|c|c|c|c|c|c|c|}
\hline$\#$ & Arm & $\alpha_{0}$ & $\alpha_{1}$ & $\alpha_{2}$ & $\alpha_{3}$ & $\alpha_{4}$ & Nonadherence & $\theta_{0}$ & $\theta_{1}$ & $\theta_{2}$ & $\theta_{3}$ & $\theta_{4}$ & $\theta_{5}$ \\
\hline \multirow{2}{*}{1} & $Z=1$ & 0.55 & 0.25 & 0.02 & 0.04 & 0.05 & 11 & 0.35 & -0.2 & 0.02 & 0.05 & 0.05 & 0 \\
\hline & $Z=0$ & 0.02 & 0.25 & 0.02 & 0.04 & 0.05 & 11 & 0.35 & -0.2 & 0.02 & 0.05 & 0.05 & 0 \\
\hline \multirow{2}{*}{2} & $Z=1$ & 0.46 & 0.25 & 0.02 & 0.04 & 0.05 & 20 & 0.35 & -0.2 & 0.02 & 0.05 & 0.05 & 0 \\
\hline & $Z=0$ & 0.12 & 0.25 & 0.02 & 0.04 & 0.05 & 21 & 0.35 & -0.2 & 0.02 & 0.05 & 0.05 & 0 \\
\hline \multirow{2}{*}{3} & $Z=1$ & 0.25 & 0.25 & 0.02 & 0.04 & 0.05 & 41 & 0.35 & -0.2 & 0.02 & 0.05 & 0.05 & 0 \\
\hline & $Z=0$ & 0.32 & 0.25 & 0.02 & 0.04 & 0.05 & 41 & 0.35 & -0.2 & 0.02 & 0.05 & 0.05 & 0 \\
\hline
\end{tabular}


Table 6 Parameterization of simulation for data generating process for simulation setting 2 outlines in DAG 2 (Exclusion restriction satisfied, unmeasured confounding present) (Continued)

\begin{tabular}{|c|c|c|c|c|c|c|c|c|c|c|c|c|c|}
\hline$\#$ & Arm & $\alpha_{0}$ & $\alpha_{1}$ & $\alpha_{2}$ & $\alpha_{3}$ & $\alpha_{4}$ & Nonadherence & $\theta_{0}$ & $\theta_{1}$ & $\theta_{2}$ & $\theta_{3}$ & $\theta_{4}$ & $\theta_{5}$ \\
\hline \multirow{2}{*}{4} & $Z=1$ & 0.05 & 0.25 & 0.02 & 0.04 & 0.05 & 61 & 0.35 & -0.2 & 0.02 & 0.05 & 0.05 & 0 \\
\hline & $Z=0$ & 0.52 & 0.25 & 0.02 & 0.04 & 0.05 & 61 & 0.35 & -0.2 & 0.02 & 0.05 & 0.05 & 0 \\
\hline \multirow{2}{*}{5} & $Z=1$ & -0.15 & 0.25 & 0.02 & 0.04 & 0.05 & 81 & 0.35 & -0.2 & 0.02 & 0.05 & 0.05 & 0 \\
\hline & $Z=0$ & 0.7 & 0.25 & 0.02 & 0.04 & 0.05 & 80 & 0.35 & -0.2 & 0.02 & 0.05 & 0.05 & 0 \\
\hline \multirow{2}{*}{6} & $Z=1$ & -0.25 & 0.25 & 0.02 & 0.04 & 0.05 & 91 & 0.35 & -0.2 & 0.02 & 0.05 & 0.05 & 0 \\
\hline & $Z=0$ & 0.8 & 0.25 & 0.02 & 0.04 & 0.05 & 89 & 0.35 & -0.2 & 0.02 & 0.05 & 0.05 & 0 \\
\hline \multirow{2}{*}{7} & $Z=1$ & 0.55 & 0.25 & 0.02 & 0.04 & 0.05 & 11 & 0.2 & -0.2 & 0.02 & 0.05 & 0.4 & 0 \\
\hline & $Z=0$ & 0.02 & 0.25 & 0.02 & 0.04 & 0.05 & 11 & 0.2 & -0.2 & 0.02 & 0.05 & 0.4 & 0 \\
\hline \multirow{2}{*}{8} & $Z=1$ & 0.46 & 0.25 & 0.02 & 0.04 & 0.05 & 20 & 0.2 & -0.2 & 0.02 & 0.05 & 0.4 & 0 \\
\hline & $Z=0$ & 0.12 & 0.25 & 0.02 & 0.04 & 0.05 & 21 & 0.2 & -0.2 & 0.02 & 0.05 & 0.4 & 0 \\
\hline \multirow{2}{*}{9} & $Z=1$ & 0.25 & 0.25 & 0.02 & 0.04 & 0.05 & 41 & 0.2 & -0.2 & 0.02 & 0.05 & 0.4 & 0 \\
\hline & $Z=0$ & 0.32 & 0.25 & 0.02 & 0.04 & 0.05 & 41 & 0.2 & -0.2 & 0.02 & 0.05 & 0.4 & 0 \\
\hline \multirow{2}{*}{10} & $Z=1$ & 0.05 & 0.25 & 0.02 & 0.04 & 0.05 & 61 & 0.2 & -0.2 & 0.02 & 0.05 & 0.4 & 0 \\
\hline & $Z=0$ & 0.52 & 0.25 & 0.02 & 0.04 & 0.05 & 61 & 0.2 & -0.2 & 0.02 & 0.05 & 0.4 & 0 \\
\hline \multirow{2}{*}{11} & $Z=1$ & -0.15 & 0.25 & 0.02 & 0.04 & 0.05 & 81 & 0.2 & -0.2 & 0.02 & 0.05 & 0.4 & 0 \\
\hline & $Z=0$ & 0.7 & 0.25 & 0.02 & 0.04 & 0.05 & 80 & 0.2 & -0.2 & 0.02 & 0.05 & 0.4 & 0 \\
\hline \multirow{2}{*}{12} & $Z=1$ & -0.25 & 0.25 & 0.02 & 0.04 & 0.05 & 91 & 0.2 & -0.2 & 0.02 & 0.05 & 0.4 & 0 \\
\hline & $Z=0$ & 0.8 & 0.25 & 0.02 & 0.04 & 0.05 & 89 & 0.2 & -0.2 & 0.02 & 0.05 & 0.4 & 0 \\
\hline \multirow{2}{*}{13} & $Z=1$ & 0.55 & 0.25 & 0.02 & 0.04 & 0.05 & 11 & 0.3 & -0.05 & 0.02 & 0.05 & 0.05 & 0 \\
\hline & $Z=0$ & 0.02 & 0.25 & 0.02 & 0.04 & 0.05 & 11 & 0.3 & -0.05 & 0.02 & 0.05 & 0.05 & 0 \\
\hline \multirow{2}{*}{14} & $Z=1$ & 0.46 & 0.25 & 0.02 & 0.04 & 0.05 & 20 & 0.3 & -0.05 & 0.02 & 0.05 & 0.05 & 0 \\
\hline & $Z=0$ & 0.12 & 0.25 & 0.02 & 0.04 & 0.05 & 21 & 0.3 & -0.05 & 0.02 & 0.05 & 0.05 & 0 \\
\hline \multirow{2}{*}{15} & $Z=1$ & 0.25 & 0.25 & 0.02 & 0.04 & 0.05 & 41 & 0.3 & -0.05 & 0.02 & 0.05 & 0.05 & 0 \\
\hline & $Z=0$ & 0.32 & 0.25 & 0.02 & 0.04 & 0.05 & 41 & 0.3 & -0.05 & 0.02 & 0.05 & 0.05 & 0 \\
\hline \multirow{2}{*}{16} & $Z=1$ & 0.05 & 0.25 & 0.02 & 0.04 & 0.05 & 61 & 0.3 & -0.05 & 0.02 & 0.05 & 0.05 & 0 \\
\hline & $Z=0$ & 0.52 & 0.25 & 0.02 & 0.04 & 0.05 & 61 & 0.3 & -0.05 & 0.02 & 0.05 & 0.05 & 0 \\
\hline \multirow{2}{*}{17} & $Z=1$ & -0.15 & 0.25 & 0.02 & 0.04 & 0.05 & 81 & 0.3 & -0.05 & 0.02 & 0.05 & 0.05 & 0 \\
\hline & $Z=0$ & 0.7 & 0.25 & 0.02 & 0.04 & 0.05 & 80 & 0.3 & -0.05 & 0.02 & 0.05 & 0.05 & 0 \\
\hline \multirow{2}{*}{18} & $Z=1$ & -0.25 & 0.25 & 0.02 & 0.04 & 0.05 & 91 & 0.3 & -0.05 & 0.02 & 0.05 & 0.05 & 0 \\
\hline & $Z=0$ & 0.8 & 0.25 & 0.02 & 0.04 & 0.05 & 89 & 0.3 & -0.05 & 0.02 & 0.05 & 0.05 & 0 \\
\hline \multirow{2}{*}{19} & $Z=1$ & 0.55 & 0.25 & 0.02 & 0.04 & 0.05 & 11 & 0.15 & -0.05 & 0.02 & 0.05 & 0.4 & 0 \\
\hline & $Z=0$ & 0.02 & 0.25 & 0.02 & 0.04 & 0.05 & 11 & 0.15 & -0.05 & 0.02 & 0.05 & 0.4 & 0 \\
\hline \multirow{2}{*}{20} & $Z=1$ & 0.46 & 0.25 & 0.02 & 0.04 & 0.05 & 20 & 0.15 & -0.05 & 0.02 & 0.05 & 0.4 & 0 \\
\hline & $Z=0$ & 0.12 & 0.25 & 0.02 & 0.04 & 0.05 & 21 & 0.15 & -0.05 & 0.02 & 0.05 & 0.4 & 0 \\
\hline \multirow{2}{*}{21} & $Z=1$ & 0.25 & 0.25 & 0.02 & 0.04 & 0.05 & 41 & 0.15 & -0.05 & 0.02 & 0.05 & 0.4 & 0 \\
\hline & $Z=0$ & 0.32 & 0.25 & 0.02 & 0.04 & 0.05 & 41 & 0.15 & -0.05 & 0.02 & 0.05 & 0.4 & 0 \\
\hline \multirow{2}{*}{22} & $Z=1$ & 0.05 & 0.25 & 0.02 & 0.04 & 0.05 & 61 & 0.15 & -0.05 & 0.02 & 0.05 & 0.4 & 0 \\
\hline & $Z=0$ & 0.52 & 0.25 & 0.02 & 0.04 & 0.05 & 61 & 0.15 & -0.05 & 0.02 & 0.05 & 0.4 & 0 \\
\hline \multirow{2}{*}{23} & $Z=1$ & -0.15 & 0.25 & 0.02 & 0.04 & 0.05 & 81 & 0.15 & -0.05 & 0.02 & 0.05 & 0.4 & 0 \\
\hline & $Z=0$ & 0.7 & 0.25 & 0.02 & 0.04 & 0.05 & 80 & 0.15 & -0.05 & 0.02 & 0.05 & 0.4 & 0 \\
\hline \multirow{2}{*}{24} & $Z=1$ & -0.25 & 0.25 & 0.02 & 0.04 & 0.05 & 91 & 0.15 & -0.05 & 0.02 & 0.05 & 0.4 & 0 \\
\hline & $Z=0$ & 0.8 & 0.25 & 0.02 & 0.04 & 0.05 & 89 & 0.15 & -0.05 & 0.02 & 0.05 & 0.4 & 0 \\
\hline
\end{tabular}


Table 6 Parameterization of simulation for data generating process for simulation setting 2 outlines in DAG 2 (Exclusion restriction satisfied, unmeasured confounding present) (Continued)

\begin{tabular}{|c|c|c|c|c|c|c|c|c|c|c|c|c|c|}
\hline$\#$ & Arm & $\alpha_{0}$ & $\alpha_{1}$ & $\alpha_{2}$ & $\alpha_{3}$ & $\alpha_{4}$ & Nonadherence & $\theta_{0}$ & $\theta_{1}$ & $\theta_{2}$ & $\theta_{3}$ & $\theta_{4}$ & $\theta_{5}$ \\
\hline \multirow{2}{*}{25} & $Z=1$ & 0.55 & 0.25 & 0.02 & 0.04 & 0.05 & 11 & 0.28 & 0 & 0.02 & 0.05 & 0.05 & 0 \\
\hline & $Z=0$ & 0.02 & 0.25 & 0.02 & 0.04 & 0.05 & 11 & 0.28 & 0 & 0.02 & 0.05 & 0.05 & 0 \\
\hline \multirow{2}{*}{26} & $Z=1$ & 0.46 & 0.25 & 0.02 & 0.04 & 0.05 & 20 & 0.28 & 0 & 0.02 & 0.05 & 0.05 & 0 \\
\hline & $Z=0$ & 0.12 & 0.25 & 0.02 & 0.04 & 0.05 & 21 & 0.28 & 0 & 0.02 & 0.05 & 0.05 & 0 \\
\hline \multirow{2}{*}{27} & $Z=1$ & 0.25 & 0.25 & 0.02 & 0.04 & 0.05 & 41 & 0.28 & 0 & 0.02 & 0.05 & 0.05 & 0 \\
\hline & $Z=0$ & 0.32 & 0.25 & 0.02 & 0.04 & 0.05 & 41 & 0.28 & 0 & 0.02 & 0.05 & 0.05 & 0 \\
\hline \multirow{2}{*}{28} & $Z=1$ & 0.05 & 0.25 & 0.02 & 0.04 & 0.05 & 61 & 0.28 & 0 & 0.02 & 0.05 & 0.05 & 0 \\
\hline & $Z=0$ & 0.52 & 0.25 & 0.02 & 0.04 & 0.05 & 61 & 0.28 & 0 & 0.02 & 0.05 & 0.05 & 0 \\
\hline \multirow{2}{*}{29} & $Z=1$ & -0.15 & 0.25 & 0.02 & 0.04 & 0.05 & 81 & 0.28 & 0 & 0.02 & 0.05 & 0.05 & 0 \\
\hline & $Z=0$ & 0.7 & 0.25 & 0.02 & 0.04 & 0.05 & 80 & 0.28 & 0 & 0.02 & 0.05 & 0.05 & 0 \\
\hline \multirow{2}{*}{30} & $Z=1$ & -0.25 & 0.25 & 0.02 & 0.04 & 0.05 & 91 & 0.28 & 0 & 0.02 & 0.05 & 0.05 & 0 \\
\hline & $Z=0$ & 0.8 & 0.25 & 0.02 & 0.04 & 0.05 & 89 & 0.28 & 0 & 0.02 & 0.05 & 0.05 & 0 \\
\hline \multirow{2}{*}{31} & $Z=1$ & 0.55 & 0.25 & 0.02 & 0.04 & 0.05 & 11 & 0.13 & 0 & 0.02 & 0.05 & 0.4 & 0 \\
\hline & $Z=0$ & 0.02 & 0.25 & 0.02 & 0.04 & 0.05 & 11 & 0.13 & 0 & 0.02 & 0.05 & 0.4 & 0 \\
\hline \multirow{2}{*}{32} & $Z=1$ & 0.46 & 0.25 & 0.02 & 0.04 & 0.05 & 20 & 0.13 & 0 & 0.02 & 0.05 & 0.4 & 0 \\
\hline & $Z=0$ & 0.12 & 0.25 & 0.02 & 0.04 & 0.05 & 21 & 0.13 & 0 & 0.02 & 0.05 & 0.4 & 0 \\
\hline \multirow{2}{*}{33} & $Z=1$ & 0.25 & 0.25 & 0.02 & 0.04 & 0.05 & 41 & 0.13 & 0 & 0.02 & 0.05 & 0.4 & 0 \\
\hline & $Z=0$ & 0.32 & 0.25 & 0.02 & 0.04 & 0.05 & 41 & 0.13 & 0 & 0.02 & 0.05 & 0.4 & 0 \\
\hline \multirow{2}{*}{34} & $Z=1$ & 0.05 & 0.25 & 0.02 & 0.04 & 0.05 & 61 & 0.13 & 0 & 0.02 & 0.05 & 0.4 & 0 \\
\hline & $Z=0$ & 0.52 & 0.25 & 0.02 & 0.04 & 0.05 & 61 & 0.13 & 0 & 0.02 & 0.05 & 0.4 & 0 \\
\hline \multirow{2}{*}{35} & $Z=1$ & -0.15 & 0.25 & 0.02 & 0.04 & 0.05 & 81 & 0.13 & 0 & 0.02 & 0.05 & 0.4 & 0 \\
\hline & $Z=0$ & 0.7 & 0.25 & 0.02 & 0.04 & 0.05 & 80 & 0.13 & 0 & 0.02 & 0.05 & 0.4 & 0 \\
\hline \multirow{2}{*}{36} & $Z=1$ & -0.25 & 0.25 & 0.02 & 0.04 & 0.05 & 91 & 0.13 & 0 & 0.02 & 0.05 & 0.4 & 0 \\
\hline & $Z=0$ & 0.8 & 0.25 & 0.02 & 0.04 & 0.05 & 89 & 0.13 & 0 & 0.02 & 0.05 & 0.4 & 0 \\
\hline \multirow{2}{*}{37} & $Z=1$ & 0.55 & 0.25 & 0.02 & 0.04 & 0.05 & 11 & 0.25 & 0.05 & 0.02 & 0.05 & 0.05 & 0 \\
\hline & $Z=0$ & 0.02 & 0.25 & 0.02 & 0.04 & 0.05 & 11 & 0.25 & 0.05 & 0.02 & 0.05 & 0.05 & 0 \\
\hline \multirow{2}{*}{38} & $Z=1$ & 0.46 & 0.25 & 0.02 & 0.04 & 0.05 & 20 & 0.25 & 0.05 & 0.02 & 0.05 & 0.05 & 0 \\
\hline & $Z=0$ & 0.12 & 0.25 & 0.02 & 0.04 & 0.05 & 21 & 0.25 & 0.05 & 0.02 & 0.05 & 0.05 & 0 \\
\hline \multirow{2}{*}{39} & $Z=1$ & 0.25 & 0.25 & 0.02 & 0.04 & 0.05 & 41 & 0.25 & 0.05 & 0.02 & 0.05 & 0.05 & 0 \\
\hline & $Z=0$ & 0.32 & 0.25 & 0.02 & 0.04 & 0.05 & 41 & 0.25 & 0.05 & 0.02 & 0.05 & 0.05 & 0 \\
\hline \multirow{2}{*}{40} & $Z=1$ & 0.05 & 0.25 & 0.02 & 0.04 & 0.05 & 61 & 0.25 & 0.05 & 0.02 & 0.05 & 0.05 & 0 \\
\hline & $Z=0$ & 0.52 & 0.25 & 0.02 & 0.04 & 0.05 & 61 & 0.25 & 0.05 & 0.02 & 0.05 & 0.05 & 0 \\
\hline \multirow{2}{*}{41} & $Z=1$ & -0.15 & 0.25 & 0.02 & 0.04 & 0.05 & 81 & 0.25 & 0.05 & 0.02 & 0.05 & 0.05 & 0 \\
\hline & $Z=0$ & 0.7 & 0.25 & 0.02 & 0.04 & 0.05 & 80 & 0.25 & 0.05 & 0.02 & 0.05 & 0.05 & 0 \\
\hline \multirow{2}{*}{42} & $Z=1$ & -0.25 & 0.25 & 0.02 & 0.04 & 0.05 & 91 & 0.25 & 0.05 & 0.02 & 0.05 & 0.05 & 0 \\
\hline & $Z=0$ & 0.8 & 0.25 & 0.02 & 0.04 & 0.05 & 89 & 0.25 & 0.05 & 0.02 & 0.05 & 0.05 & 0 \\
\hline \multirow{2}{*}{43} & $Z=1$ & 0.55 & 0.25 & 0.02 & 0.04 & 0.05 & 11 & 0.1 & 0.05 & 0.02 & 0.05 & 0.4 & 0 \\
\hline & $Z=0$ & 0.02 & 0.25 & 0.02 & 0.04 & 0.05 & 11 & 0.1 & 0.05 & 0.02 & 0.05 & 0.4 & 0 \\
\hline \multirow{2}{*}{44} & $Z=1$ & 0.46 & 0.25 & 0.02 & 0.04 & 0.05 & 20 & 0.1 & 0.05 & 0.02 & 0.05 & 0.4 & 0 \\
\hline & $Z=0$ & 0.12 & 0.25 & 0.02 & 0.04 & 0.05 & 21 & 0.1 & 0.05 & 0.02 & 0.05 & 0.4 & 0 \\
\hline \multirow{2}{*}{45} & $Z=1$ & 0.25 & 0.25 & 0.02 & 0.04 & 0.05 & 41 & 0.1 & 0.05 & 0.02 & 0.05 & 0.4 & 0 \\
\hline & $Z=0$ & 0.32 & 0.25 & 0.02 & 0.04 & 0.05 & 41 & 0.1 & 0.05 & 0.02 & 0.05 & 0.4 & 0 \\
\hline
\end{tabular}


Table 6 Parameterization of simulation for data generating process for simulation setting 2 outlines in DAG 2 (Exclusion restriction satisfied, unmeasured confounding present) (Continued)

\begin{tabular}{|c|c|c|c|c|c|c|c|c|c|c|c|c|c|}
\hline$\#$ & Arm & $\alpha_{0}$ & $\alpha_{1}$ & $\alpha_{2}$ & $\alpha_{3}$ & $\alpha_{4}$ & Nonadherence & $\theta_{0}$ & $\theta_{1}$ & $\theta_{2}$ & $\theta_{3}$ & $\theta_{4}$ & $\theta_{5}$ \\
\hline \multirow{2}{*}{46} & $Z=1$ & 0.05 & 0.25 & 0.02 & 0.04 & 0.05 & 61 & 0.1 & 0.05 & 0.02 & 0.05 & 0.4 & 0 \\
\hline & $Z=0$ & 0.52 & 0.25 & 0.02 & 0.04 & 0.05 & 61 & 0.1 & 0.05 & 0.02 & 0.05 & 0.4 & 0 \\
\hline \multirow{2}{*}{47} & $Z=1$ & -0.15 & 0.25 & 0.02 & 0.04 & 0.05 & 81 & 0.1 & 0.05 & 0.02 & 0.05 & 0.4 & 0 \\
\hline & $Z=0$ & 0.7 & 0.25 & 0.02 & 0.04 & 0.05 & 80 & 0.1 & 0.05 & 0.02 & 0.05 & 0.4 & 0 \\
\hline \multirow{2}{*}{48} & $Z=1$ & -0.25 & 0.25 & 0.02 & 0.04 & 0.05 & 91 & 0.1 & 0.05 & 0.02 & 0.05 & 0.4 & 0 \\
\hline & $Z=0$ & 0.8 & 0.25 & 0.02 & 0.04 & 0.05 & 89 & 0.1 & 0.05 & 0.02 & 0.05 & 0.4 & 0 \\
\hline \multirow{2}{*}{49} & $Z=1$ & 0.55 & 0.25 & 0.02 & 0.04 & 0.05 & 11 & 0.2 & 0.2 & 0.02 & 0.05 & 0.05 & 0 \\
\hline & $Z=0$ & 0.02 & 0.25 & 0.02 & 0.04 & 0.05 & 11 & 0.2 & 0.2 & 0.02 & 0.05 & 0.05 & 0 \\
\hline \multirow{2}{*}{50} & $Z=1$ & 0.46 & 0.25 & 0.02 & 0.04 & 0.05 & 20 & 0.2 & 0.2 & 0.02 & 0.05 & 0.05 & 0 \\
\hline & $Z=0$ & 0.12 & 0.25 & 0.02 & 0.04 & 0.05 & 21 & 0.2 & 0.2 & 0.02 & 0.05 & 0.05 & 0 \\
\hline \multirow{2}{*}{51} & $Z=1$ & 0.25 & 0.25 & 0.02 & 0.04 & 0.05 & 41 & 0.2 & 0.2 & 0.02 & 0.05 & 0.05 & 0 \\
\hline & $Z=0$ & 0.32 & 0.25 & 0.02 & 0.04 & 0.05 & 41 & 0.2 & 0.2 & 0.02 & 0.05 & 0.05 & 0 \\
\hline \multirow{2}{*}{52} & $Z=1$ & 0.05 & 0.25 & 0.02 & 0.04 & 0.05 & 61 & 0.2 & 0.2 & 0.02 & 0.05 & 0.05 & 0 \\
\hline & $Z=0$ & 0.52 & 0.25 & 0.02 & 0.04 & 0.05 & 61 & 0.2 & 0.2 & 0.02 & 0.05 & 0.05 & 0 \\
\hline \multirow{2}{*}{53} & $Z=1$ & -0.15 & 0.25 & 0.02 & 0.04 & 0.05 & 81 & 0.2 & 0.2 & 0.02 & 0.05 & 0.05 & 0 \\
\hline & $Z=0$ & 0.7 & 0.25 & 0.02 & 0.04 & 0.05 & 80 & 0.2 & 0.2 & 0.02 & 0.05 & 0.05 & 0 \\
\hline \multirow{2}{*}{54} & $Z=1$ & -0.25 & 0.25 & 0.02 & 0.04 & 0.05 & 91 & 0.2 & 0.2 & 0.02 & 0.05 & 0.05 & 0 \\
\hline & $Z=0$ & 0.8 & 0.25 & 0.02 & 0.04 & 0.05 & 89 & 0.2 & 0.2 & 0.02 & 0.05 & 0.05 & 0 \\
\hline \multirow{2}{*}{55} & $Z=1$ & 0.55 & 0.25 & 0.02 & 0.04 & 0.05 & 11 & 0.02 & 0.2 & 0.02 & 0.05 & 0.4 & 0 \\
\hline & $Z=0$ & 0.02 & 0.25 & 0.02 & 0.04 & 0.05 & 11 & 0.02 & 0.2 & 0.02 & 0.05 & 0.4 & 0 \\
\hline \multirow{2}{*}{56} & $Z=1$ & 0.46 & 0.25 & 0.02 & 0.04 & 0.05 & 20 & 0.02 & 0.2 & 0.02 & 0.05 & 0.4 & 0 \\
\hline & $Z=0$ & 0.12 & 0.25 & 0.02 & 0.04 & 0.05 & 21 & 0.02 & 0.2 & 0.02 & 0.05 & 0.4 & 0 \\
\hline \multirow{2}{*}{57} & $Z=1$ & 0.25 & 0.25 & 0.02 & 0.04 & 0.05 & 41 & 0.02 & 0.2 & 0.02 & 0.05 & 0.4 & 0 \\
\hline & $Z=0$ & 0.32 & 0.25 & 0.02 & 0.04 & 0.05 & 41 & 0.02 & 0.2 & 0.02 & 0.05 & 0.4 & 0 \\
\hline \multirow{2}{*}{58} & $Z=1$ & 0.05 & 0.25 & 0.02 & 0.04 & 0.05 & 61 & 0.02 & 0.2 & 0.02 & 0.05 & 0.4 & 0 \\
\hline & $Z=0$ & 0.52 & 0.25 & 0.02 & 0.04 & 0.05 & 61 & 0.02 & 0.2 & 0.02 & 0.05 & 0.4 & 0 \\
\hline \multirow{2}{*}{59} & $Z=1$ & -0.15 & 0.25 & 0.02 & 0.04 & 0.05 & 81 & 0.02 & 0.2 & 0.02 & 0.05 & 0.4 & 0 \\
\hline & $Z=0$ & 0.7 & 0.25 & 0.02 & 0.04 & 0.05 & 80 & 0.02 & 0.2 & 0.02 & 0.05 & 0.4 & 0 \\
\hline \multirow{2}{*}{60} & $Z=1$ & -0.25 & 0.25 & 0.02 & 0.04 & 0.05 & 91 & 0.02 & 0.2 & 0.02 & 0.05 & 0.4 & 0 \\
\hline & $Z=0$ & 0.8 & 0.25 & 0.02 & 0.04 & 0.05 & 89 & 0.02 & 0.2 & 0.02 & 0.05 & 0.4 & 0 \\
\hline
\end{tabular}

Table 7 Parameterization of simulation for data generating process for simulation setting 3 outlines in DAG 3 (Exclusion restriction violated)

\begin{tabular}{|c|c|c|c|c|c|c|c|c|c|c|c|c|c|}
\hline$\#$ & Arm & $\alpha_{0}$ & $\alpha_{1}$ & $\alpha_{2}$ & $\alpha_{3}$ & $\alpha_{4}$ & Nonadherence & $\theta_{0}$ & $\theta_{1}$ & $\theta_{2}$ & $\theta_{3}$ & $\theta_{4}$ & $\theta_{5}$ \\
\hline \multirow{2}{*}{1} & $Z=1$ & 0.86 & 0 & 0.01 & 0.04 & 0 & 10 & 0.2 & 0 & 0.03 & 0.1 & 0.05 & 0.05 \\
\hline & $Z=0$ & 0.06 & 0 & 0.01 & 0.04 & 0 & 10 & 0.2 & 0 & 0.03 & 0.1 & 0.05 & 0.05 \\
\hline \multirow{2}{*}{2} & $Z=1$ & 0.76 & 0 & 0.01 & 0.04 & 0 & 20 & 0.2 & 0 & 0.03 & 0.1 & 0.05 & 0.05 \\
\hline & $Z=0$ & 0.16 & 0 & 0.01 & 0.04 & 0 & 20 & 0.2 & 0 & 0.03 & 0.1 & 0.05 & 0.05 \\
\hline \multirow{2}{*}{3} & $Z=1$ & 0.56 & 0 & 0.01 & 0.04 & 0 & 41 & 0.2 & 0 & 0.03 & 0.1 & 0.05 & 0.05 \\
\hline & $Z=0$ & 0.36 & 0 & 0.01 & 0.04 & 0 & 40 & 0.2 & 0 & 0.03 & 0.1 & 0.05 & 0.05 \\
\hline \multirow{2}{*}{4} & $Z=1$ & 0.36 & 0 & 0.01 & 0.04 & 0 & 60 & 0.2 & 0 & 0.03 & 0.1 & 0.05 & 0.05 \\
\hline & $Z=0$ & 0.57 & 0 & 0.01 & 0.04 & 0 & 60 & 0.2 & 0 & 0.03 & 0.1 & 0.05 & 0.05 \\
\hline
\end{tabular}


Table 7 Parameterization of simulation for data generating process for simulation setting 3 outlines in DAG 3 (Exclusion restriction violated) (Continued)

\begin{tabular}{|c|c|c|c|c|c|c|c|c|c|c|c|c|c|}
\hline$\#$ & Arm & $\alpha_{0}$ & $\alpha_{1}$ & $\alpha_{2}$ & $\alpha_{3}$ & $\alpha_{4}$ & Nonadherence & $\theta_{0}$ & $\theta_{1}$ & $\theta_{2}$ & $\theta_{3}$ & $\theta_{4}$ & $\theta_{5}$ \\
\hline \multirow{2}{*}{5} & $Z=1$ & 0.16 & 0 & 0.01 & 0.04 & 0 & 80 & 0.2 & 0 & 0.03 & 0.1 & 0.05 & 0.05 \\
\hline & $Z=0$ & 0.77 & 0 & 0.01 & 0.04 & 0 & 80 & 0.2 & 0 & 0.03 & 0.1 & 0.05 & 0.05 \\
\hline \multirow{2}{*}{6} & $Z=1$ & 0.06 & 0 & 0.01 & 0.04 & 0 & 90 & 0.2 & 0 & 0.03 & 0.1 & 0.05 & 0.05 \\
\hline & $Z=0$ & 0.86 & 0 & 0.01 & 0.04 & 0 & 90 & 0.2 & 0 & 0.03 & 0.1 & 0.05 & 0.05 \\
\hline \multirow{2}{*}{7} & $Z=1$ & 0.86 & 0 & 0.01 & 0.04 & 0 & 10 & 0.1 & 0 & 0.03 & 0.1 & 0.05 & 0.2 \\
\hline & $Z=0$ & 0.06 & 0 & 0.01 & 0.04 & 0 & 10 & 0.1 & 0 & 0.03 & 0.1 & 0.05 & 0.2 \\
\hline \multirow{2}{*}{8} & $Z=1$ & 0.76 & 0 & 0.01 & 0.04 & 0 & 20 & 0.1 & 0 & 0.03 & 0.1 & 0.05 & 0.2 \\
\hline & $Z=0$ & 0.16 & 0 & 0.01 & 0.04 & 0 & 20 & 0.1 & 0 & 0.03 & 0.1 & 0.05 & 0.2 \\
\hline \multirow{2}{*}{9} & $Z=1$ & 0.56 & 0 & 0.01 & 0.04 & 0 & 41 & 0.1 & 0 & 0.03 & 0.1 & 0.05 & 0.2 \\
\hline & $Z=0$ & 0.36 & 0 & 0.01 & 0.04 & 0 & 40 & 0.1 & 0 & 0.03 & 0.1 & 0.05 & 0.2 \\
\hline \multirow{2}{*}{10} & $Z=1$ & 0.36 & 0 & 0.01 & 0.04 & 0 & 60 & 0.1 & 0 & 0.03 & 0.1 & 0.05 & 0.2 \\
\hline & $Z=0$ & 0.57 & 0 & 0.01 & 0.04 & 0 & 60 & 0.1 & 0 & 0.03 & 0.1 & 0.05 & 0.2 \\
\hline \multirow{2}{*}{11} & $Z=1$ & 0.16 & 0 & 0.01 & 0.04 & 0 & 80 & 0.1 & 0 & 0.03 & 0.1 & 0.05 & 0.2 \\
\hline & $Z=0$ & 0.77 & 0 & 0.01 & 0.04 & 0 & 80 & 0.1 & 0 & 0.03 & 0.1 & 0.05 & 0.2 \\
\hline \multirow{2}{*}{12} & $Z=1$ & 0.06 & 0 & 0.01 & 0.04 & 0 & 90 & 0.1 & 0 & 0.03 & 0.1 & 0.05 & 0.2 \\
\hline & $Z=0$ & 0.86 & 0 & 0.01 & 0.04 & 0 & 90 & 0.1 & 0 & 0.03 & 0.1 & 0.05 & 0.2 \\
\hline \multirow{2}{*}{13} & $Z=1$ & 0.86 & 0 & 0.01 & 0.04 & 0 & 10 & 0.2 & 0.2 & 0.03 & 0.1 & 0.05 & 0.05 \\
\hline & $Z=0$ & 0.06 & 0 & 0.01 & 0.04 & 0 & 10 & 0.2 & 0.2 & 0.03 & 0.1 & 0.05 & 0.05 \\
\hline \multirow{2}{*}{14} & $Z=1$ & 0.76 & 0 & 0.01 & 0.04 & 0 & 20 & 0.2 & 0.2 & 0.03 & 0.1 & 0.05 & 0.05 \\
\hline & $Z=0$ & 0.16 & 0 & 0.01 & 0.04 & 0 & 20 & 0.2 & 0.2 & 0.03 & 0.1 & 0.05 & 0.05 \\
\hline \multirow{2}{*}{15} & $Z=1$ & 0.56 & 0 & 0.01 & 0.04 & 0 & 41 & 0.2 & 0.2 & 0.03 & 0.1 & 0.05 & 0.05 \\
\hline & $Z=0$ & 0.36 & 0 & 0.01 & 0.04 & 0 & 40 & 0.2 & 0.2 & 0.03 & 0.1 & 0.05 & 0.05 \\
\hline \multirow{2}{*}{16} & $Z=1$ & 0.36 & 0 & 0.01 & 0.04 & 0 & 60 & 0.2 & 0.2 & 0.03 & 0.1 & 0.05 & 0.05 \\
\hline & $Z=0$ & 0.57 & 0 & 0.01 & 0.04 & 0 & 60 & 0.2 & 0.2 & 0.03 & 0.1 & 0.05 & 0.05 \\
\hline \multirow{2}{*}{17} & $Z=1$ & 0.16 & 0 & 0.01 & 0.04 & 0 & 80 & 0.2 & 0.2 & 0.03 & 0.1 & 0.05 & 0.05 \\
\hline & $Z=0$ & 0.77 & 0 & 0.01 & 0.04 & 0 & 80 & 0.2 & 0.2 & 0.03 & 0.1 & 0.05 & 0.05 \\
\hline \multirow{2}{*}{18} & $Z=1$ & 0.06 & 0 & 0.01 & 0.04 & 0 & 90 & 0.2 & 0.2 & 0.03 & 0.1 & 0.05 & 0.05 \\
\hline & $Z=0$ & 0.86 & 0 & 0.01 & 0.04 & 0 & 90 & 0.2 & 0.2 & 0.03 & 0.1 & 0.05 & 0.05 \\
\hline \multirow{2}{*}{19} & $Z=1$ & 0.86 & 0 & 0.01 & 0.04 & 0 & 10 & 0.1 & 0.2 & 0.03 & 0.1 & 0.05 & 0.2 \\
\hline & $Z=0$ & 0.06 & 0 & 0.01 & 0.04 & 0 & 10 & 0.1 & 0.2 & 0.03 & 0.1 & 0.05 & 0.2 \\
\hline \multirow{2}{*}{20} & $Z=1$ & 0.76 & 0 & 0.01 & 0.04 & 0 & 20 & 0.1 & 0.2 & 0.03 & 0.1 & 0.05 & 0.2 \\
\hline & $Z=0$ & 0.16 & 0 & 0.01 & 0.04 & 0 & 20 & 0.1 & 0.2 & 0.03 & 0.1 & 0.05 & 0.2 \\
\hline \multirow{2}{*}{21} & $Z=1$ & 0.56 & 0 & 0.01 & 0.04 & 0 & 41 & 0.1 & 0.2 & 0.03 & 0.1 & 0.05 & 0.2 \\
\hline & $Z=0$ & 0.36 & 0 & 0.01 & 0.04 & 0 & 40 & 0.1 & 0.2 & 0.03 & 0.1 & 0.05 & 0.2 \\
\hline \multirow{2}{*}{22} & $Z=1$ & 0.36 & 0 & 0.01 & 0.04 & 0 & 60 & 0.1 & 0.2 & 0.03 & 0.1 & 0.05 & 0.2 \\
\hline & $Z=0$ & 0.57 & 0 & 0.01 & 0.04 & 0 & 60 & 0.1 & 0.2 & 0.03 & 0.1 & 0.05 & 0.2 \\
\hline \multirow{2}{*}{23} & $Z=1$ & 0.16 & 0 & 0.01 & 0.04 & 0 & 80 & 0.1 & 0.2 & 0.03 & 0.1 & 0.05 & 0.2 \\
\hline & $Z=0$ & 0.77 & 0 & 0.01 & 0.04 & 0 & 80 & 0.1 & 0.2 & 0.03 & 0.1 & 0.05 & 0.2 \\
\hline \multirow{2}{*}{24} & $Z=1$ & 0.06 & 0 & 0.01 & 0.04 & 0 & 90 & 0.1 & 0.2 & 0.03 & 0.1 & 0.05 & 0.2 \\
\hline & $Z=0$ & 0.86 & 0 & 0.01 & 0.04 & 0 & 90 & 0.1 & 0.2 & 0.03 & 0.1 & 0.05 & 0.2 \\
\hline
\end{tabular}




\section{Abbreviations}

2SLS: Two-stage least square; 2SRI: Two-stage residual inclusion; AT: As-treated; Cl: Confidence interval; DAG: Directed acyclic graph; IP-weighted PP: Inverse probability-weighted per-protocol; ITT: Intention-to-treat; IV: Instrumental variable; MSE: Mean squared error; NPCB: Nonparametric causal bound; PP: Per-protocol; RD: Risk difference; SE: Standard error

\section{Acknowledgements}

Authors thank the anonymous reviewers for their constructive comments, which helped improve the manuscript. Authors would like to acknowledge Ali Shajarizadeh for his valuable comments on an earlier version of the draft manuscript. We also thank Sharon Roman, a patient partner of the BC SUPPORT Unit methods clusters, for her involvement with the project.

\section{Authors' contributions}

MBH performed the simulations and analysis, drafted the initial manuscript, and revised it based on received comments. LM initially assessed the feasibility of the work via a different set of simulations that informed some directions of this work, critically reviewed the manuscript, and provided comments. MEK conceptualized the research question, secured funding, supervised the research, critically reviewed and revised the manuscript. All authors read and approved the final manuscript.

\section{Funding}

This work was supported by BC Support Unit's Real-World Clinical Trials Methods Cluster, Project \#2, led by Dr. Karim (with research members Paul Gustafson, Joan Hu, Hubert Wong and Derek Ouyang), and Dr. Karim's Natural Sciences and Engineering Research Council of Canada (NSERC) fund and NSERC Discovery Accelerator Supplements (PG\#: $20 R 01603$ and 20R12709; PI: M.E.K.).

\section{Availability of data and materials}

The dataset used during the current study is available from the corresponding author upon request.

\section{Declarations}

\section{Ethics approval and consent to participate}

Not applicable.

\section{Consent for publication}

Not applicable.

\section{Competing interests}

$\mathrm{MBH}$ and LM declare no potential conflict of interests. MEK is supported by the Michael Smith Foundation for Health Research Scholar award (ID\#: 17661). Over the past three years, MEK has received consulting fees from Biogen Inc. for consulting in projects unrelated to the current project.

\section{Author details}

${ }^{1}$ School of Population and Public Health, University of British Columbia, Vancouver, BC, Canada. ${ }^{2}$ Department of Statistics, University of British Columbia, Vancouver, BC, Canada. ${ }^{3}$ Centre for Health Evaluation and Outcome Sciences, St. Paul's Hospital, Vancouver, BC, Canada.

\section{Received: 13 April 2021 Accepted: 14 January 2022}

Published online: 16 February 2022

\section{References}

1. Schwartz D, Lellouch J. Explanatory and pragmatic attitudes in therapeutical trials. J Chronic Dis. 1967;20(8):637-48. https://doi.org/10. 1016/j.jclinepi.2009.01.012.

2. Ford I, Norrie J. Pragmatic trials. N Engl J Med. 2016;375(5):454-63. https://doi.org/10.1056/NEJMra1510059.

3. Chalmers TC, Celano P, Sacks HS, Smith Jr H. Bias in treatment assignment in controlled clinical trials. N Engl J Med. 1983;309(22): 1358-61. https://doi.org/10.1056/NEJM198312013092204.

4. Hernan MA, Robins JM. Per-protocol analyses of pragmatic trials. N Engl J Med. 2017;377(14):1391-8.

5. Ware $\mathrm{JH}$, Hamel MB. Pragmatic trials-guides to better patient care. N Engl J Med. 2011;364(18):1685-7. https://doi.org/10.1056/NEJMp1103502.

6. Gupta SK. Intention-to-treat concept: a review. Perspect Clin Res. 2011;2(3):109. https://doi.org/10.4103/2229-3485.83221.
7. Toh S, Hernandez-Diaz S, Logan R, Robins JM, Hernan MA. Estimating absolute risks in the presence of nonadherence: an application to a follow-up study with baseline randomization. Epidemiology. 2010;21(4): 528.

8. Hernan MA, Scharfstein D. Cautions as regulators move to end exclusive reliance on intention to treat. Ann Intern Med. 2018. https://doi.org/10. 7326/M17-3354.

9. Hernan MA, Hernandez-Diaz S. Beyond the intention-to-treat in comparative effectiveness research. Clin Trials. 2012;9(1):48-55.

10. Cook JA, MacLennan GS, Palmer T, Lois N, Emsley R. Instrumental variable methods for a binary outcome were used to informatively address noncompliance in a randomized trial in surgery. J Clin Epidemiol. 2018;96:126-32. https://doi.org/10.1016/j.jclinepi.2017.11.011.

11. Chlebowski RT, Anderson GL, Gass M, Lane DS, Aragaki AK, Kuller LH, Manson JE, Stefanick ML, Ockene J, Sarto GE. Estrogen plus progestin and breast cancer incidence and mortality in postmenopausal women. J Am Med Assoc. 2010;304(15):1684-92. https://doi.org/10.1001/jama. 2010.1500.

12. Blewer AL, Putt ME, Becker LB, Riegel BJ, Li J, Leary M, Shea JA, Kirkpatrick JN, Berg RA, Nadkarni VM. Video-only cardiopulmonary resuscitation education for high-risk families before hospital discharge: a multicenter pragmatic trial. Circ Cardiovasc Qual Outcomes. 2016;9(6): 740-8. https://doi.org/10.1161/CIRCOUTCOMES.116.002493.

13. Mash RJ, Rhode H, Zwarenstein M, Rollnick S, Lombard C, Steyn K, Levitt N. Effectiveness of a group diabetes education programme in under-served communities in south africa: a pragmatic cluster randomized controlled trial. Diabet Med. 2014;31(8):987-93. https://doi. org/10.1111/dme.12475.

14. Little RJ, Long Q, Lin X. A comparison of methods for estimating the causal effect of a treatment in randomized clinical trials subject to noncompliance. Biometrics. 2009;65(2):640-9.

15. Baiocchi M, Cheng J, Small DS. Instrumental variable methods for causal inference. Stat Med. 2014;33(13):2297-340. https://doi.org/10.1002/sim. 6128.

16. Lobban F, Akers N, Appelbe D, Chapman L, Collinge L, Dodd S, Flowers $\mathrm{S}$, Hollingsworth B, Johnson S, Jones SH. Clinical effectiveness of a web-based peer-supported self-management intervention for relatives of people with psychosis or bipolar (react): online, observer-blind, randomised controlled superiority trial. BMC Psychiatry. 2020;20:1-16. https://doi.org/10.1186/s12888-020-02545-9.

17. Powell J, Williams V, Atherton $H$, Bennett $K$, Yang $Y$, Davoudianfar M, Hellsing A, Martin A, Mollison J, Shanyinde M. Effectiveness and cost-effectiveness of a self-guided internet intervention for social anxiety symptoms in a general population sample: Randomized controlled trial. J Med Internet Res. 2020;22(1):16804. https://doi.org/10.2196/16804.

18. Manski CF. Nonparametric bounds on treatment effects. Am Econ Rev. 1990;80(2):319-23.

19. Palmer TM, Ramsahai RR, Didelez V, Sheehan NA. Nonparametric bounds for the causal effect in a binary instrumental-variable model. Stata J. 2011;11(3):345-67. https://doi.org/10.1177/1536867X1101100302.

20. Balke A, Pearl J. Bounds on treatment effects from studies with imperfect compliance. J Am Stat Assoc. 1997;92(439):1171-6.

21. Murray EJ, Hernan MA. Improved adherence adjustment in the coronary drug project. Trials. 2018;19(1):158. https://doi.org/10.1186/s13063-0182519-5.

22. Terza VJ. Two-stage residual inclusion estimation in health services research and health economics. Health Serv Res. 2018;53(3):1890-9. https://doi.org/10.1111/1475-6773.12714.

23. Rassen JA, Schneeweiss S, Glynn RJ, Mittleman MA, Brookhart MA. Instrumental variable analysis for estimation of treatment effects with dichotomous outcomes. Am J Epidemiol. 2009;169(3):273-84. https://doi. org/10.1093/aje/kwn299.

24. Angrist JD, Imbens GW, Rubin DB. Identification of causal effects using instrumental variables. J Am Stat Assoc. 1996;91(434):444-55.

25. Danaei G, Rodríguez LAG, Cantero OF, Logan R, Hernan MA. Observational data for comparative effectiveness research: an emulation of randomised trials of statins and primary prevention of coronary heart disease. Stat Methods Med Res. 2013;22(1):70-96.

26. Young JG, Vatsa R, Murray EJ, Hernan MA. Interval-cohort designs and bias in the estimation of per-protocol effects: a simulation study. Trials. 2019;20(1):552.

27. Mosquera L. Exploring inverse probability weighted per-protocol estimates to adjust for non-adherence using post-randomization 
covariates: a simulation study MSc thesis, University of British Columbia. 2020. http://hdl.handle.net/2429/75673.

28. Garrido MM, Deb P, Burgess Jr JF, Penrod JD. Choosing models for health care cost analyses: issues of nonlinearity and endogeneity. Health Serv Res. 2012;47(6):2377-97.

29. Wang F, Meyer NJ, Walley KR, Russell JA, Feng R. Causal genetic inference using haplotypes as instrumental variables. Genet Epidemiol. 2016;40(1):35-44.

30. Hernan MA, Robins JM. Estimating causal effects from epidemiological data. J Epidemiol Community Health. 2006;60(7):578-86. https://doi.org/ 10.1136/jech.2004.029496.

31. Dunn G, Maracy M, Tomenson B. Estimating treatment effects from randomized clinical trials with noncompliance and loss to follow-up: the role of instrumental variable methods. Stat Methods Med Res. 2005;14(4): 369-95. https://doi.org/10.1191/0962280205sm403oa.

32. McNamee R. Confounding and confounders. Occup Environ Med. 2003;60(3):227-34.

33. Lederer DJ, Bell SC, Branson RD, Chalmers JD, Marshall R, Maslove DM, Ost DE, Punjabi NM, Schatz M, Smyth AR, et al. Control of confounding and reporting of results in causal inference studies. guidance for authors from editors of respiratory, sleep, and critical care journals. Ann Am Thorac Soc. 2019;16(1):22-8.

34. VanderWeele TJ. Principles of confounder selection. Eur J Epidemiol. 2019:34(3):211-9.

35. Textor J, van der Zander B, Gilthorpe MS, Liśkiewicz M, Ellison GT. Robust causal inference using directed acyclic graphs: the r package 'dagitty'. Int J Epidemiol. 2016;45(6):1887-94.

36. Hernán MA, Robins JM. Instruments for causal inference: an epidemiologist's dream?. Epidemiology. 2006;17(4):360-72.

37. Brookhart MA, Wang P, Solomon DH, Schneeweiss S. Evaluating short-term drug effects using a physician-specific prescribing preference as an instrumental variable. Epidemiology. 2006;17(3):268.

38. Greenland S, Pearl J, Robins JM. Confounding and collapsibility in causal inference. Stat Sci. 1999;14(1):29-46.

39. Kaufman JS. Marginalia: comparing adjusted effect measures. Epidemiology. 2010;21(4):490-3.

40. Naimi Al, Whitcomb BW. Estimating risk ratios and risk differences using regression. Am J Epidemiol. 2020;189(6):508-10.

41. Booth FW, Roberts CK, Laye MJ. Lack of exercise is a major cause of chronic diseases. Compr Physiol. 2012;2(2):1143.

42. Garcia-Aymerich J, Lange P, Benet M, Schnohr P, Antó JM. Regular physical activity modifies smoking-related lung function decline and reduces risk of chronic obstructive pulmonary disease: a population-based cohort study. Am J Respir Crit Care Med. 2007;175(5):458-63.

43. Crothers K, Griffith TA, McGinnis KA, Rodriguez-Barradas MC, Leaf DA, Weissman S, Gibert CL, Butt AA, Justice AC. The impact of cigarette smoking on mortality, quality of life, and comorbid illness among hiv-positive veterans. J Gen Intern Med. 2005;20(12):1142-5.

44. Cohen S, Tyrrell D, Russell MA, Jarvis MJ, Smith AP. Smoking, alcohol consumption, and susceptibility to the common cold. Am J Public Health. 1993;83(9):1277-83.

45. Schmid P, Rauber D, Betsch C, Lidolt G, Denker M-L. Barriers of influenza vaccination intention and behavior-a systematic review of influenza vaccine hesitancy, 2005-2016. Plos ONE. 2017;12(1):0170550.

46. Hirano K, Imbens GW, Rubin DB, Zhou X-H. Assessing the effect of an influenza vaccine in an encouragement design. Biostatistics. 2000;1 (1): 69-88.

47. Morris TP, White IR, Crowther MJ. Using simulation studies to evaluate statistical methods. Stat Med. 2019;38(11):2074-102. https://doi.org/10. 1002/sim.8086.

48. Sommer A, Djunaedi E, Loeden A, Tarwotjo I, West JR K, Tilden R, Mele L, Group AS, et al. Impact of vitamin A supplementation on childhood mortality: a randomised controlled community trial. Lancet. 1986;327(8491):1169-73.

49. Sommer A, Zeger SL. On estimating efficacy from clinical trials. Stat Med. 1991;10(1):45-52.

50. McNamee R. Intention to treat, per protocol, as treated and instrumental variable estimators given non-compliance and effect heterogeneity. Stat Med. 2009;28(21):2639-52.

51. Bang H, Davis CE. On estimating treatment effects under non-compliance in randomized clinical trials: are intent-to-treat or instrumental variables analyses perfect solutions?. Stat Med. 2007;26(5):954-64.

52. Nagelkerke N, Fidler $V$, Bernsen R, Borgdorff M. Estimating treatment effects in randomized clinical trials in the presence of non-compliance. Stat Med. 2000;19(14):1849-64.
53. NICE. Guide to the methods of technology appraisal. Technical report. 2013.

54. Hernán MA, Robins JM. Confounding. In: Causal Inference: What If. Boca Raton: Chapman and Hall/CRC; 2020

55. Terza VJ, Basu A, Rathouz PJ. Two-stage residual inclusion estimation: addressing endogeneity in health econometric modeling. J Health Econ. 2008;27(3):531-43. https://doi.org/10.1016/j.jhealeco.2007.09.009.

56. Bound J, Jaeger DA, Baker RM. Problems with instrumental variables estimation when the correlation between the instruments and the endogenous explanatory variable is weak. J Am Stat Assoc. 1995;90(430): 443-50. https://doi.org/10.2307/2291055.

57. Small DS, Rosenbaum PR. War and wages: the strength of instrumental variables and their sensitivity to unobserved biases. J Am Stat Assoc. 2008;103(483):924-33. https://doi.org/10.1198/016214507000001247.

58. Cole SR, Hernan MA. Constructing inverse probability weights for marginal structural models. Am J Epidemiol. 2008;168(6):656-64. https:// doi.org/10.1093/aje/kwn164.

59. Hernán MA, Hernández-Díaz S, Robins JM. A structural approach to selection bias. Epidemiology. 2004;15(5):615-25.

60. Moerbeek M, Schie S. v. What are the statistical implications of treatment non-compliance in cluster randomized trials: a simulation study. Stat Med. 2019:38(26):5071-84.

61. Ye C, Beyene J, Browne G, Thabane L. Estimating treatment effects in randomised controlled trials with non-compliance: a simulation study. BMJ Open. 2014;4(6):005362.

62. Gao X, Zheng M. Estimating the causal effects in randomized trials for survival data with a cure fraction and non compliance. Commun Stat Theory Methods. 2017;46(8):4065-87.

63. Nie H, Cheng J, Small DS. Inference for the effect of treatment on survival probability in randomized trials with noncompliance and administrative censoring. Biometrics. 2011;67(4):1397-405.

64. Latimer N, Abrams K, Siebert U. Two-stage estimation to adjust for treatment switching in randomised trials: a simulation study investigating the use of inverse probability weighting instead of re-censoring. BMC Med Res Methodol. 2019;19(1):1-19.

65. Cai B, Small DS, Have TRT. Two-stage instrumental variable methods for estimating the causal odds ratio: analysis of bias. Stat Med. 2011;30(15): 1809-24.

66. Uddin M, Groenwold R, de Boer Ton A., Belitser S, Roes K, Klungel O. Instrumental variable analysis in epidemiologic studies: an overview of the estimation methods. Pharma Analytica Acta. 2015;6(353):2.

67. Robins J, Rotnitzky A. Estimation of treatment effects in randomised trials with non-compliance and a dichotomous outcome using structural mean models. Biometrika. 2004;91(4):763-83.

68. Westreich D, Cole SR. Positivity in practice. Am J Epidemiol. 2010;171(6): 674-7.

69. Platt RW, Delaney JAC, Suissa S. The positivity assumption and marginal structural models: the example of warfarin use and risk of bleeding. Eur J Epidemiol. 2012;27(2):77-83.

70. Crump RK, Hotz VJ, Imbens GW, Mitnik OA. Dealing with limited overlap in estimation of average treatment effects. Biometrika. 2009;96(1):187-99.

71. Li F, Morgan KL, Zaslavsky AM. Balancing covariates via propensity score weighting. J Am Stat Assoc. 2018;113(521):390-400.

72. Lee Y, Kennedy EH, Mitra N. Doubly robust nonparametric instrumental variable estimators for survival outcomes. Biostatistics. 2021. Published online: 17 Oct 2021. https://doi.org/10.1093/biostatistics/kxab036.

73. Choi BY. Instrumental variable estimation of truncated local average treatment effects. Plos ONE. 2021;16(4):0249642.

74. Cole SR, Frangakis CE. The consistency statement in causal inference: a definition or an assumption?. Epidemiology. 2009;20(1):3-5. https://doi. org/10.1097/EDE.0b013e31818ef366.

75. Hernan MA. Beyond exchangeability: the other conditions for causal inference in medical research. Stat Methods Med Res. 2012;21(1):3-5. https://doi.org/10.1177/0962280211398037.

76. Vansteelandt S, Bekaert M, Claeskens G. On model selection and model misspecification in causal inference. Stat Methods Med Res. 2012;21(1): 7-30. https://doi.org/10.1177/0962280210387717.

77. Brookhart MA, Rassen JA, Schneeweiss S. Instrumental variable methods in comparative safety and effectiveness research. Pharmacoepidemiol Drug Saf. 2010;19(6):537-54. https://doi.org/10.1002/pds.1908.

78. Hernan MA, Robins JM. Selection bias. In: Causal Inference: What If. Boca Raton: Chapman and Hall/CRC; 2020

\section{Publisher's Note}

Springer Nature remains neutral with regard to jurisdictional claims in published maps and institutional affiliations. 